\title{
Fourier Coefficients of a Class of Eta Quotients of Weight 16 with Level 12
}

\author{
Barış Kendirli \\ Istanbul Kultur University, Istanbul, Turkey \\ Email: baris.kendirli@gmail.com
}

Received 30 May 2015; accepted 28 July 2015; published 31 July 2015

Copyright (C) 2015 by author and Scientific Research Publishing Inc.

This work is licensed under the Creative Commons Attribution International License (CC BY). http://creativecommons.org/licenses/by/4.0/

(c) (i) Open Access

\section{Abstract}

Recently, Williams [1] and then Yao, Xia and Jin [2] discovered explicit formulas for the coefficients of the Fourier series expansions of a class of eta quotients. Williams expressed all coefficients of 126 eta quotients in terms of $\sigma(n), \sigma\left(\frac{n}{2}\right), \sigma\left(\frac{n}{3}\right)$ and $\sigma\left(\frac{n}{6}\right)$ and Yao, Xia and Jin, following the method of proof of Williams, expressed only even coefficients of 104 eta quotients in terms of $\sigma_{3}(n), \sigma_{3}\left(\frac{n}{2}\right), \sigma_{3}\left(\frac{n}{3}\right)$ and $\sigma_{3}\left(\frac{n}{6}\right)$. Here, by using the method of proof of Williams, we will express the even Fourier coefficients of 360 eta quotients i.e., the Fourier coefficients of the sum, $f(q)+f(-q)$, of 360 eta quotients in terms of $\sigma_{15}(n), \sigma_{15}\left(\frac{n}{2}\right), \sigma_{15}\left(\frac{n}{3}\right), \sigma_{15}\left(\frac{n}{4}\right), \sigma_{15}\left(\frac{n}{6}\right)$ and $\sigma_{15}\left(\frac{n}{12}\right)$

\section{Keywords}

Dedekind Eta Function, Eta Quotients, Fourier Series

The divisor function $\sigma_{i}(n)$ is defined for a positive integer $i$ by

$$
\begin{aligned}
& \sigma_{i}(n):=\sum_{d \text { positive integer, } d \mid n} d^{i} \text {,if } n \text { is a positive integer, and } \\
& \sigma_{i}(n):=0 \text { if } n \text { is not a positive integer. }
\end{aligned}
$$

The Dedekind eta function is defined by 


$$
\eta(z):=q^{1 / 24} \prod_{n=1}^{\infty}\left(1-q^{n}\right)
$$

where

$$
q:=\mathrm{e}^{2 \pi i z}, z \in H=\{x+i y: y>0\}
$$

And an eta quotient of level $n$ is defined by

$$
f(z):=\prod_{m \mid n} \eta(m z)^{a_{m}}, n \in \mathbb{N}, a_{m} \in Z, a_{n} \neq 0 .
$$

It is interesting and important to determine explicit formulas of the Fourier coefficients of eta quotients since they are the building blocks of modular forms of level $n$ and weight $k$. The book of Köhler [3] (Chapter 3, p. 39) describes such expansions by means of Hecke Theta series and develops algorithms for the determination of suitable eta quotients. One can find more information in [4]-[8]. I have determined the Fourier coefficients of the theta series associated to some quadratic forms, see [9]-[14].

Recently, Williams, see [1] discovered explicit formulas for the coefficients of Fourier series expansions of a class of 126 eta quotients in terms of $\sigma(n), \sigma\left(\frac{n}{2}\right), \sigma\left(\frac{n}{3}\right)$ and $\sigma\left(\frac{n}{6}\right)$. One example is as follows:

$$
\frac{\eta^{2}(2 z) \eta^{4}(4 z) \eta^{6}(6 z)}{\eta^{2}(z) \eta^{2}(3 z) \eta^{4}(12 z)}
$$

gives the expansion found by Williams.

Then Yao, Xia and Jin [2] expressed the even Fourier coefficients of 104 eta quotients in terms of $\sigma_{3}(n), \sigma_{3}\left(\frac{n}{2}\right), \sigma_{3}\left(\frac{n}{3}\right)$ and $\sigma_{3}\left(\frac{n}{6}\right)$. One example is as follows:

$$
\frac{\eta^{25}(2 z) \eta^{4}(3 z)}{\eta^{12}(z) \eta^{5}(4 z) \eta^{3}(6 z) \eta(12 z)}
$$

where the even coefficients are obtained. Motivated by these two results, we find that we can express the even Fourier coefficients of 360 eta quotients in terms of $\sigma_{15}(n), \sigma_{15}\left(\frac{n}{2}\right), \sigma_{15}\left(\frac{n}{3}\right), \sigma_{15}\left(\frac{n}{4}\right), \sigma_{15}\left(\frac{n}{6}\right)$ and $\sigma_{15}\left(\frac{n}{12}\right)$, see Table 2. One example is as follows:

$$
\frac{\eta^{12}(4 z) \eta^{10}(6 z) \eta^{4}(12 z)}{\eta^{6}(2 z)}
$$

We see that the odd Fourier coefficients of 875 eta quotients are zero and even coefficients can be expressed by simple formula. Let

$$
\begin{aligned}
& f_{1}=\frac{\eta^{18}(4 z) \eta^{16}(6 z) \eta^{10}(12 z)}{\eta^{12}(2 z)}, \\
& f_{2}=\frac{\eta^{20}(4 z) \eta^{2}(6 z) \eta^{20}(12 z)}{\eta^{10}(2 z)}, \\
& f_{3}=\frac{\eta^{15}(4 z) \eta^{7}(6 z) \eta^{19}(12 z)}{\eta^{9}(2 z)}, \\
& f_{4}=\frac{\eta^{10}(2 z) \eta^{12}(4 z) \eta^{18}(6 z)}{\eta^{8}(12 z)},
\end{aligned}
$$




$$
\begin{aligned}
& f_{5}=\frac{\eta^{5}(4 z) \eta^{17}(6 z) \eta^{17}(12 z)}{\eta^{7}(2 z)}, \\
& f_{6}=\frac{\eta^{17}(4 z) \eta^{17}(6 z) \eta^{5}(12 z)}{\eta^{7}(2 z)}, \\
& f_{7}=\frac{\eta^{19}(4 z) \eta^{15}(6 z) \eta^{15}(12 z)}{\eta^{17}(2 z)}, \\
& f_{8}=\frac{\eta^{17}(2 z) \eta^{17}(4 z) \eta^{5}(12 z)}{\eta^{7}(6 z)} \\
& f_{9}=\frac{\eta^{20}(2 z) \eta^{14}(4 z) \eta^{14}(12 z)}{\eta^{16}(6 z)}, \\
& f_{10}=\frac{\eta^{16}(4 z) \eta^{18}(6 z)}{\eta^{2}(2 z)}, \\
& f_{11}=\frac{\eta^{20}(2 z) \eta^{14}(4 z) \eta^{8}(6 z)}{\eta^{10}(12 z)}, \\
& f_{12}=\eta^{20}(2 z) \eta^{2}(4 z) \eta^{8}(6 z) \eta^{2}(12 z) \text {, } \\
& f_{13}=\frac{\eta^{2}(2 z) \eta^{14}(6 z) \eta^{20}(12 z)}{\eta^{4}(4 z)}, \\
& f_{14}=\frac{\eta^{16}(2 z) \eta^{10}(4 z) \eta^{18}(12 z)}{\eta^{12}(6 z)}, \\
& f_{15}=\frac{\eta^{19}(4 z) \eta^{9}(6 z) \eta^{15}(12 z)}{\eta^{11}(2 z)}, \\
& f_{16}=\frac{\eta^{14}(4 z) \eta^{14}(6 z) \eta^{14}(12 z)}{\eta^{10}(2 z)}, \\
& f_{17}=\frac{\eta^{15}(4 z) \eta^{13}(6 z) \eta^{19}(12 z)}{\eta^{15}(2 z)}, \\
& f_{18}=\frac{\eta^{9}(4 z) \eta^{19}(6 z) \eta^{13}(12 z)}{\eta^{9}(2 z)}, \\
& f_{19}=\frac{\eta^{18}(4 z) \eta^{10}(6 z) \eta^{10}(12 z)}{\eta^{6}(2 z)}, \\
& f_{20}=\frac{\eta^{20}(2 z) \eta^{8}(4 z) \eta^{20}(6 z)}{\eta^{16}(12 z)}, \\
& f_{21}=\frac{\eta^{20}(2 z) \eta^{20}(4 z)}{\eta^{4}(6 z) \eta^{4}(12 z)}, \\
& f_{22}=\frac{\eta^{20}(4 z) \eta^{20}(12 z)}{\eta^{4}(2 z) \eta^{4}(6 z)},
\end{aligned}
$$




$$
\begin{gathered}
f_{23}=\frac{\eta^{20}(4 z) \eta^{20}(6 z)}{\eta^{4}(2 z) \eta^{4}(12 z)}, \\
f_{24}=\frac{\eta^{15}(4 z) \eta(6 z) \eta^{19}(12 z)}{\eta^{3}(2 z)}, \\
f_{25}=\frac{\eta^{20}(2 z) \eta^{20}(4 z)}{\eta^{4}(6 z) \eta^{4}(12 z)}, \\
f_{26}=\frac{\eta^{20}(2 z) \eta^{20}(6 z) \eta^{8}(12 z)}{\eta^{4}(4 z)}, \\
f_{27}=\frac{\eta^{19}(2 z) \eta^{13}(4 z) \eta^{15}(6 z)}{\eta^{15}(12 z)} .
\end{gathered}
$$

Now we can state our main Theorem:

Theorem 1 Let $b_{1}, b_{2}, \cdots, b_{5}$ be non-negative integers satisfying

$$
b_{1}+b_{2}+\cdots+b_{5} \leq 32 \text {. }
$$

Define the integers $a_{1}, a_{2}, a_{3}, a_{4}, a_{6}, a_{12}$ by

$$
\begin{gathered}
a_{1}:=-b_{1}+2 b_{2}-2 b_{3}-4 b_{4}-b_{5}+32, \\
a_{2}:=3 b_{1}+b_{2}+3 b_{3}+10 b_{4}+b_{5}-80, \\
a_{3}:=3 b_{1}+2 b_{2}+6 b_{3}+4 b_{4}+3 b_{5}-96, \\
a_{4}:=-2 b_{1}-b_{2}-b_{3}-4 b_{4}+2 b_{5}+32, \\
a_{6}:=-9 b_{1}-7 b_{2}-9 b_{3}-10 b_{4}-7 b_{5}+240, \\
a_{12}:=6 b_{1}+3 b_{2}+3 b_{3}+4 b_{4}+2 b_{5}-96 .
\end{gathered}
$$

They are functions of $q$ by (3). Now define integers

$$
\begin{aligned}
& k_{0}, k_{1}, k_{2}, k_{3}, k_{4}, k_{5}, k_{6}, k_{7}, k_{8}, k_{9}, k_{10}, k_{11}, k_{12}, k_{13}, k_{14}, k_{15}, k_{16}, k_{17}, k_{18}, k_{19}, \\
& k_{20}, k_{21}, k_{22}, k_{23}, k_{24}, k_{25}, k_{26}, k_{27}, k_{28}, k_{29}, k_{30}, k_{31} \text { and } k_{32}
\end{aligned}
$$

by

$$
\begin{gathered}
\frac{1}{2^{b_{1}+b_{5}}} x^{b_{1}}(1-x)^{b_{2}}(1+x)^{b_{3}}(1+2 x)^{b_{4}}(2+x)^{b_{5}} \\
=k_{0}+k_{1} x+k_{2} x^{2}+k_{3} x+k_{4} x^{4}+k_{5} x^{5}+k_{6} x^{6}+k_{7} x^{7}+k_{8} x^{8} \\
+k_{9} x^{9}+k_{10} x^{10}+k_{11} x^{11}+k_{12} x^{12}+k_{13} x^{13}+k_{14} x^{14}+k_{15} x^{15} \\
+k_{16} x^{16}+k_{17} x^{17}+k_{18} x^{18}+k_{19} x^{19}+k_{20} x^{20} \\
+k_{21} x^{21}+k_{22} x^{22}+k_{23} x^{23}+k_{24} x^{24}+k_{25} x^{25}+k_{26} x^{26}+k_{27} x^{27} \\
+k_{28} x^{28}+k_{29} x^{29}+k_{30} x^{30}+k_{31} x^{31}+k_{32} x^{32} .
\end{gathered}
$$

Define the rational numbers

$$
c_{1}, c_{2}, c_{3}, c_{4}, c_{6}, c_{12}, r_{1}, r_{2}, \cdots, r_{26}
$$

and $r_{27}$ as in Table 1. Here $\left\{f_{1}, \cdots, f_{27}\right\} \backslash\left\{f_{7}, f_{9}, f_{17}, f_{20}, f_{25}, f_{26}, f_{27}\right\} \in S_{16}\left(\Gamma_{0}(12)\right)$, $f_{7}, f_{9}, f_{17}, f_{20}, f_{25}, f_{26}, f_{27} \in M_{16}\left(\Gamma_{0}(12)\right) \backslash S_{16}\left(\Gamma_{0}(12)\right)$ and 
Table 1. Coefficients of eisenstein series and some eta quotients.

$$
\begin{aligned}
& c_{1}:=\frac{72583}{980659548} k_{1}-\frac{120832}{245164887} k_{0}-\frac{2735}{326886516} k_{2}+\frac{277}{490329774} k_{3} \\
&-\frac{7}{490329774} k_{4}+\frac{1}{36320724} k_{5}-\frac{13}{245164887} k_{6}+\frac{25}{245164887} k_{7} \\
&-\frac{16}{81721629} k_{8}+\frac{92}{245164887} k_{9}-\frac{16}{22287717} k_{10}+\frac{112}{81721629} k_{11} \\
&-\frac{640}{245164887} k_{12}+\frac{4352}{245164887} k_{15}-\frac{256}{27240543} k_{14}-\frac{1216}{245164887} k_{13} \\
&-\frac{8192}{245164887} k_{16}+\frac{5120}{81721629} k_{17}-\frac{28672}{245164887} k_{18}+\frac{16384}{22287717} k_{21} \\
&-\frac{32768}{81721629} k_{20}-\frac{327680}{245164887} k_{22}+\frac{65536}{27240543} k_{23} \\
&-\frac{1048576}{245164887} k_{24}+\frac{53248}{245164887} k_{19}+\frac{1835008}{245164887} k_{25} \\
&-\frac{1048576}{81721629} k_{26}+\frac{5242880}{245164887} k_{27}-\frac{8388608}{245164887} k_{28} \\
&+\frac{4194304}{81721629} k_{29}-\frac{16777216}{245164887} k_{30}+\frac{16777216}{245164887} k_{31},
\end{aligned}
$$

$c_{2}:=\frac{34734530088732674832514185950084774049248}{2142599748818134613113093495072137244191} k_{0}-\frac{62956800237180571066473807796775564347301}{25711196985817615357357121940865646930292} k_{1}$

$+\frac{560501183955670989597137220160641262555}{1977784383524431950565932456989665148484} k_{2}-\frac{97964204168754996402004893769133989949}{4285199497636269226226186990144274488382} k_{3}$

$+\frac{40897072982474021350305265538542513037}{12855598492908807678678560970432823465146} k_{4}-\frac{77910984221605457897100564905533152137}{2571119698581761535735712194086564630292} k_{5}$

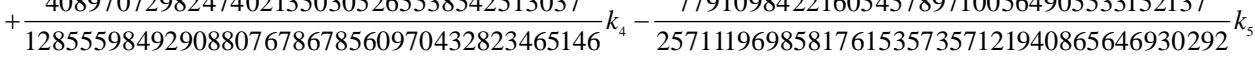

$+\frac{7683007415360393635856788341175110341}{2142599748818134613113093495072137244191} k_{6}-\frac{32060487020365187360697936804888726131}{6427799246454403839339280485216411732573} k_{7}$

$+\frac{50628602500203669592250667935460937744}{6427799246454403839339280485216411732573} k_{8}-\frac{29085265762693348400769904586321479036}{2142599748818134613113093495072137244191} k_{9}$

$+\frac{158250367120128739881846817708074446992}{6427799246454403839339280485216411732573} k_{10}-\frac{22656914712598824036276628186340738864}{494446095881107987641483114247416287121} k_{11}$

$+\frac{184848740939247688020554787142399039616}{2142599748818134613113093495072137244191} k_{12}-\frac{1048239944217959494935262133888301514304}{6427799246454403839339280485216411732573} k_{13}$

$+\frac{25174768088427596815019432883901899008}{164815365293702662547161038082472095707} k_{14}-\frac{1246723832169732120072934552513970970880}{2142599748818134613113093495072137244191} k_{15}$

$+\frac{7038115875495624397185080978321395609600}{6427799246454403839339280485216411732573} k_{16}-\frac{13195297276418057599661347671371361539072}{6427799246454403839339280485216411732573} k_{17}$

$+\frac{8210357018229573060477785588795679469568}{2142599748818134613113093495072137244191} k_{18}-\frac{45744265651265841051078471764079279534080}{6427799246454403839339280485216411732573} k_{19}$

$+\frac{84452773012339913380407176682315598299136}{6427799246454403839339280485216411732573} k_{20}-\frac{51610948844243996791857065512420047634432}{2142599748818134613113093495072137244191} k_{21}$

$+\frac{21655225465002831817250269915926736076800}{494446095881107987641483114247416287121} k_{22}-\frac{506736790874131434657008627249238597238784}{6427799246454403839339280485216411732573} k_{23}$

$+\frac{300290233988701749697945831633510766477312}{2142599748818134613113093495072137244191} k_{24}-\frac{1576529708086760827933004700454891591958528}{6427799246454403839339280485216411732573} k_{25}$

$+\frac{2702628877067406586383696467255320708907008}{6427799246454403839339280485216411732573} k_{26}-\frac{1501462766976347698189587052601574136348672}{2142599748818134613113093495072137244191} k_{27}$

$+\frac{7207028070602459124588069514635005543317504}{6427799246454403839339280485216411732573} k_{28}-\frac{10810548170070847789240155429646823984857088}{6427799246454403839339280485216411732573} k_{29}$ $+\frac{4804689379205214665850988696123019241979904}{2142599748818134613113093495072137244191} k_{30}-\frac{14414064906651085511759265915202903948132352}{6427799246454403839339280485216411732573} k_{31}$ 12614340427608524802511638703898624

$-\frac{12614340427608524802511638703898624}{1892198777290080612110474090437566009} k_{32}$ 


\section{Continued}

$$
\begin{aligned}
& c_{3}:=\frac{32}{2187} k_{0}-\frac{62}{2187} k_{1}+\frac{40}{729} k_{2}-\frac{232}{2187} k_{3}+\frac{448}{2187} k_{4} \\
& -\frac{32}{81} k_{5}+\frac{1664}{2187} k_{6}-\frac{3200}{2187} k_{7}+\frac{2048}{729} k_{8}-\frac{11776}{2187} k_{9} \\
& +\frac{22528}{2187} k_{10}-\frac{14336}{729} k_{11}+\frac{81920}{2187} k_{12}-\frac{155648}{2187} k_{13} \\
& +\frac{32768}{243} k_{14}-\frac{557056}{2187} k_{15}+\frac{1048576}{2187} k_{16}-\frac{655360}{729} k_{17} \\
& +\frac{4194304}{729} k_{20}-\frac{23068672}{2187} k_{21}+\frac{41943040}{2187} k_{22} \\
& -\frac{8388608}{243} k_{23}+\frac{134217728}{2187} k_{24}+\frac{3670016}{2187} k_{18} \\
& -\frac{6815744}{2187} k_{19}+\frac{2147483648}{2187} k_{30}+\frac{234881024}{2187} k_{25} \\
& +\frac{134217728}{729} k_{26}-\frac{671088640}{2187} k_{27}+\frac{1073741824}{2187} k_{28} \\
& -\frac{536870912}{729} k_{29}-\frac{2147483648}{2187} k_{31},
\end{aligned}
$$

$c_{4}:=-\frac{3438631669957687943317991998219836912041984}{212117375132995326698196256012141587174909} k_{0}+\frac{519391370415717958219513005709135610101760}{212117375132995326698196256012141587174909} k_{1}$ $-\frac{1541655078827786053038522300113680482304}{5438907054692187864056314256721579158331} k_{2}+\frac{4861344345762952626180655745881508651008}{212117375132995326698196256012141587174909} k_{3}$ $5438907054692187864056314256721579158331 k_{2}+212117375132995326698196256012141587174909$ $-\frac{686662260194965967174344185570009972736}{212117375132995326698196256012141587174909} k_{4}+\frac{24223505589260903505657457023611248640}{7856199078999826914748009481931169895367} k_{5}$ $-\frac{770912905776039933043040352765543514112}{212117375132995326698196256012141587174909} k_{6}+\frac{1066674040873884195145072515431390019584}{212117375132995326698196256012141587174909} k_{7}$ $\frac{558909424814416346855153477262757068800}{70705791710998444232732085337380529058303} k_{\mathrm{g}}+\frac{2880943447310005657271900824199337410560}{212117375132995326698196256012141587174909} k_{9}$ $474212116868164817910723564062331109376 k_{k}+\frac{248759939095225256330737009613353582592}{54380054692187864056314256721579158331}$ $-\frac{19283397739363211518017841455649235197719}{10}+\frac{24875903807054692187864056314256721579158331}{543891}$ $-\frac{18261794573801688749784466114240638353408}{212117375132995326698196256012141587174909} k_{12}+\frac{34521130653913088315205006307078765740032}{212117375132995326698196256012141587174909} k_{13}$ $7252972970368272989918505205394851364864 k_{14}+\frac{123219038849645502527071198852093878009856}{21211737513299532669819625601214158717490} k_{15}$ $-\frac{25298597236999480744244028445793509686101}{214}+\frac{12321903884964550252707119885209387008569}{212117375132995326698196256012141587174909} k_{15}$ $-\frac{231919667081212951123368487557465697681408}{212117375132995326698196256012141587174909} k_{16}+\frac{144967222655613673979840116872910152466432}{70705791710998442232732085337380529058303} k_{17}$ $-\frac{811966565987547701423795536752529381916672}{212117375132995326698196256012141587174909} k_{18}+\frac{1508222015438432664091595009713631746654208}{212117375132995326698196256012141587174909} k_{19}$ $-\frac{928294070025214438646146335719133007839232}{70705791710998442232732085337380529058303} k_{20}+\frac{464214257594362122128001913465962465394688}{19283397739363211518017841455649235197719} k_{21}$ $-\frac{714261708179731033246971232677038694989824}{16316721164076563592168942770164737474993} k_{22}+\frac{1857262903145106340717278218703097268338688}{23568597236999480744244028445793509686101} k_{23}$ $-\frac{29718557905407543679170452853863882287480832}{212117375132995326698196256012141587174909} k_{24}+\frac{52010743753839595169447408643587812743643136}{212117375132995326698196256012141587174909} k_{25}$ $-\frac{29721880606491798348403071893001785716637696}{70705791710998442232732085337380529058303} k_{26}+\frac{148614907659583800278219830959223630146371584}{212117375132995326698196256012141587174909} k_{27}$ $-\frac{237790053425604749045832090773182365227548672}{212117375132995326698196256012141587174909} k_{28}+\frac{118896676835312618271459427865760036870225920}{70705791710998442232732085337380529058303} k_{29}$ $-\frac{475583378556974223031298806035732201510404096}{212117375132995326698196256012141587174909} k_{30}+\frac{475544908027362753251165705288941682960629760}{212117375132995326698196256012141587174909} k_{31}$ 69662864213164369817684884330643456 $+\frac{696061436195928911559647188534724667}{7008} k_{32}$ 


\section{Continued}

$C_{6}:=-\frac{903140823425764539266175742781129563328}{1892198777290080612110474090437566009} k_{0}+\frac{1753599251608810820042292756595554516256}{1892198777290080612110474090437566009} k_{1}$ $-\frac{261382625829009040606391162572202246708}{145553752099236970162344160802889693} k_{2}+\frac{6573369312471681371367564937022266290064}{1892198777290080612110474090437566009} k_{3}$ $-\frac{12697362031501480563543217533293921843408}{1892198777290080612110474090437566009} k_{4}+\frac{24491723680826731742452810508492829778096}{1892198777290080612110474090437566009} k_{5}$

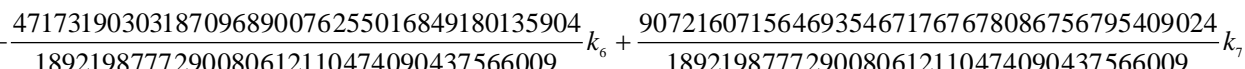

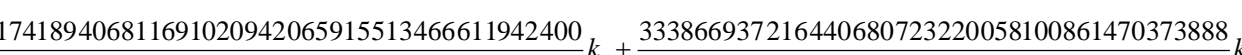

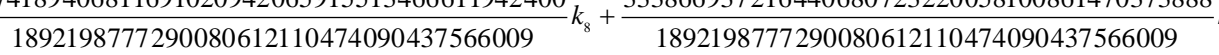
$-\frac{638705859516034234832558641988331987268352}{1892198777290080612110474090437566009} k_{10}+\frac{93796263570379824927121966639465976592640}{145553752099236970162344160802889693} k_{1}$ $-\frac{2322578004133601510600390747954985709790720}{1892198777290080612110474090437566009} k_{12}+\frac{4412902046734060157362588620252943713253888}{189219877729008061211047409043756609} k_{13}$

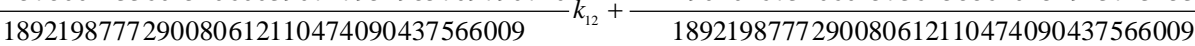
$-\frac{8361291905582645393038766948930775507203072}{1892198777290080612110474090437566009} k_{14}$ 15793555169896537058264823055463258597435392 1892198777290080612110474090437566009 $-\frac{29729048791079112416901620246445683858493440}{1892198777290080612110474090437566009} k_{1}$ $+\frac{55741970217875135338106642659799456846036992}{1892198777290080612110474090437566009} k_{17}$ 104051681439650108926296625737844180389232640 1892198777290080612110474090437566009

$+\frac{193238840618886918635723645699723731688488960}{k}$ 1892198777290080612110474090437566009 $-\frac{356748632448054978156317427837374824256110592}{1892198777290080612110474090437566009} k_{20}$ $+\frac{654039163047100217481579906467314595064709120}{1892198777290080612110474090437566009} k_{21}$ $\frac{91474009086609878519169859048974830832975872}{1455537520992369} k$ 145553752099236970162344160802889693

$+\frac{2140491816044378587791643159133715725272612864}{18921987772900800612110474090437566009} k_{23}$ 1892198777290080612110474090437566009 $\frac{3805318787402182990247254077934953884303753216}{1892198777290080612110474090437566009} k_{24}$ $+\frac{6659307881158913920815458330065825499748892672}{1892198777290080612110474090437566009} k_{25}$

$-\frac{11415956370753930456327232934139762697522380800}{16} k_{26}$ 1892198777290080612110474090437566009

$+\frac{19026593954106378493996218523841248653172670464}{1} k_{2}$ 1892198777290080612110474090437566009 30442550329135403612241707744616567638358228992 1892198777290080612110474090437566009 $+\frac{45663825495841001129663585013837084183763222528}{1892198777290080612110474090437566009} k_{29}$ 60885100662546560092483434885863179799076012032 1892198777290080612110474090437566009

$+\frac{60885100662544944606303920653576106827577294848}{1892198777290080612110474090437566009} k_{31}$ 1892198777290080612110474090437566009

$\frac{8561044434669014598204843153342595072}{1892198777290080612110474090437566009} k_{32}$, 


\section{Continued}

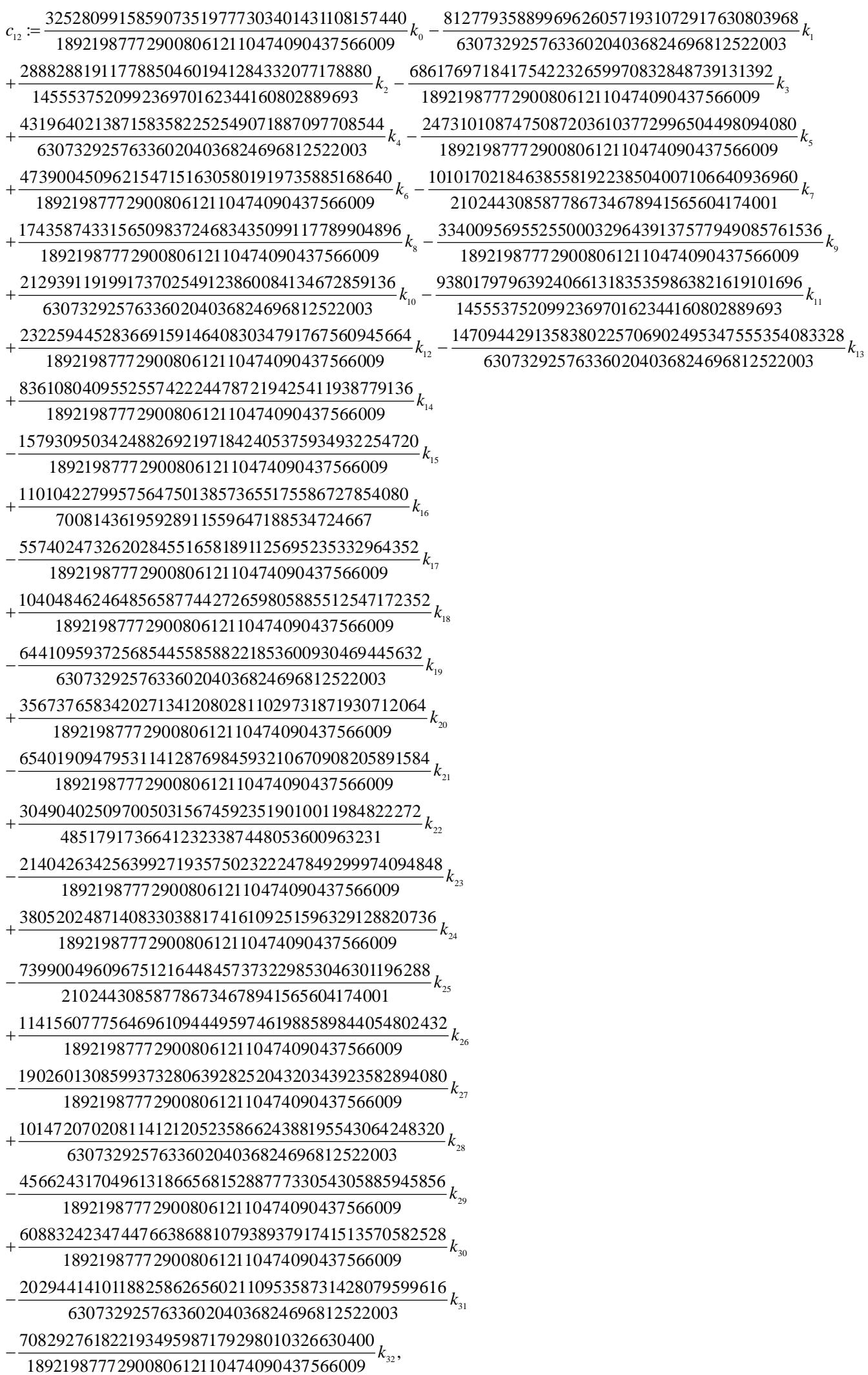




\section{Continued}

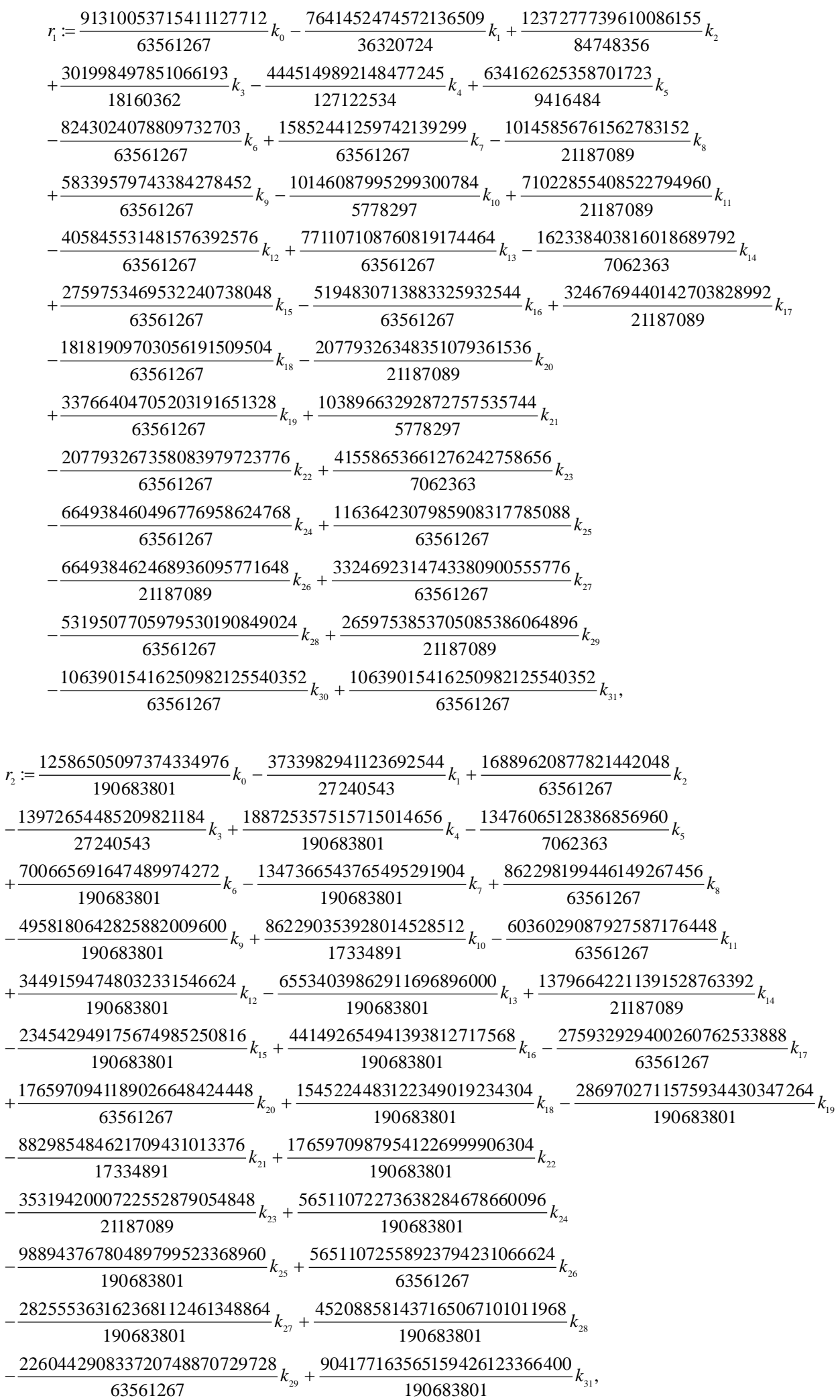




\section{Continued}

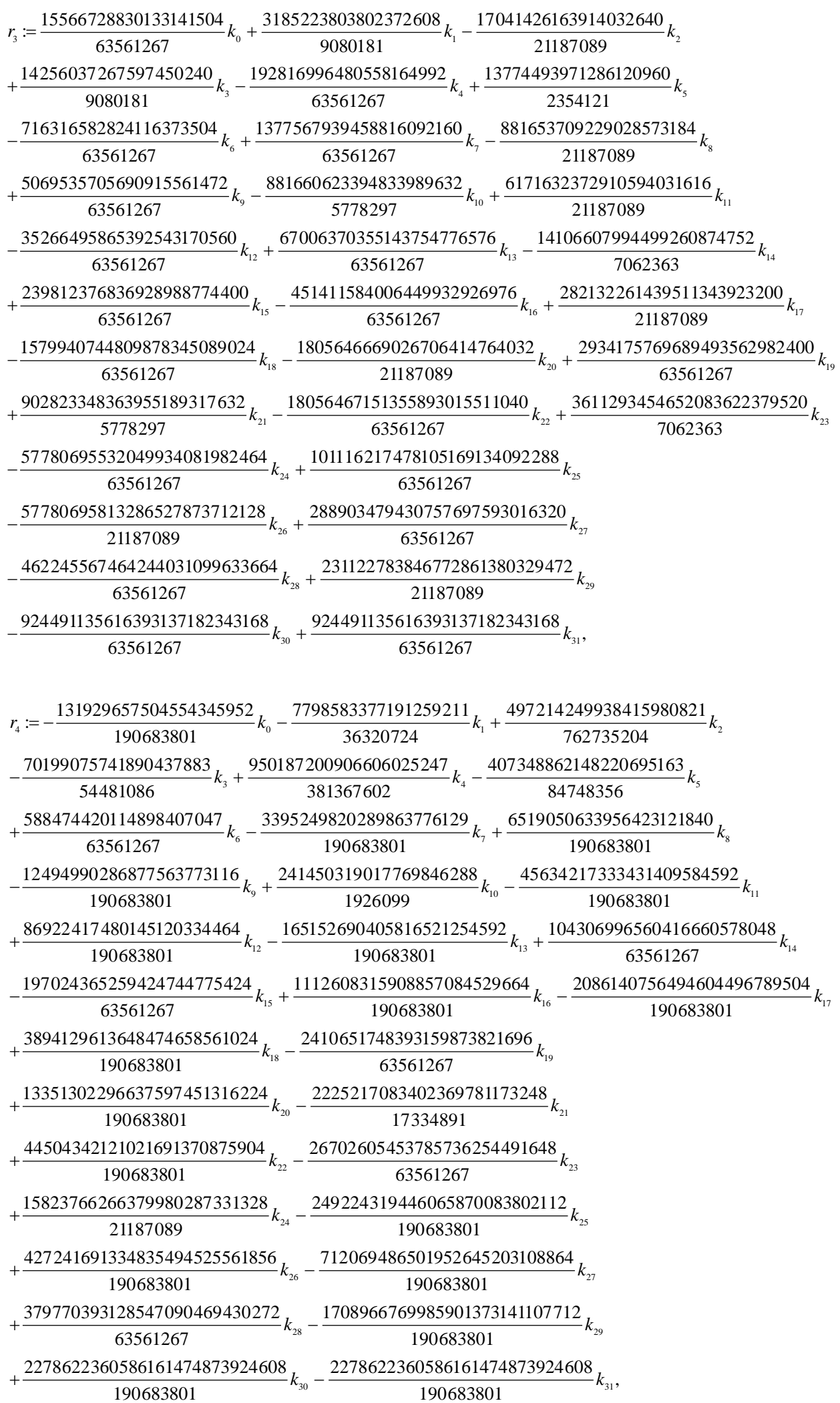




\section{Continued}

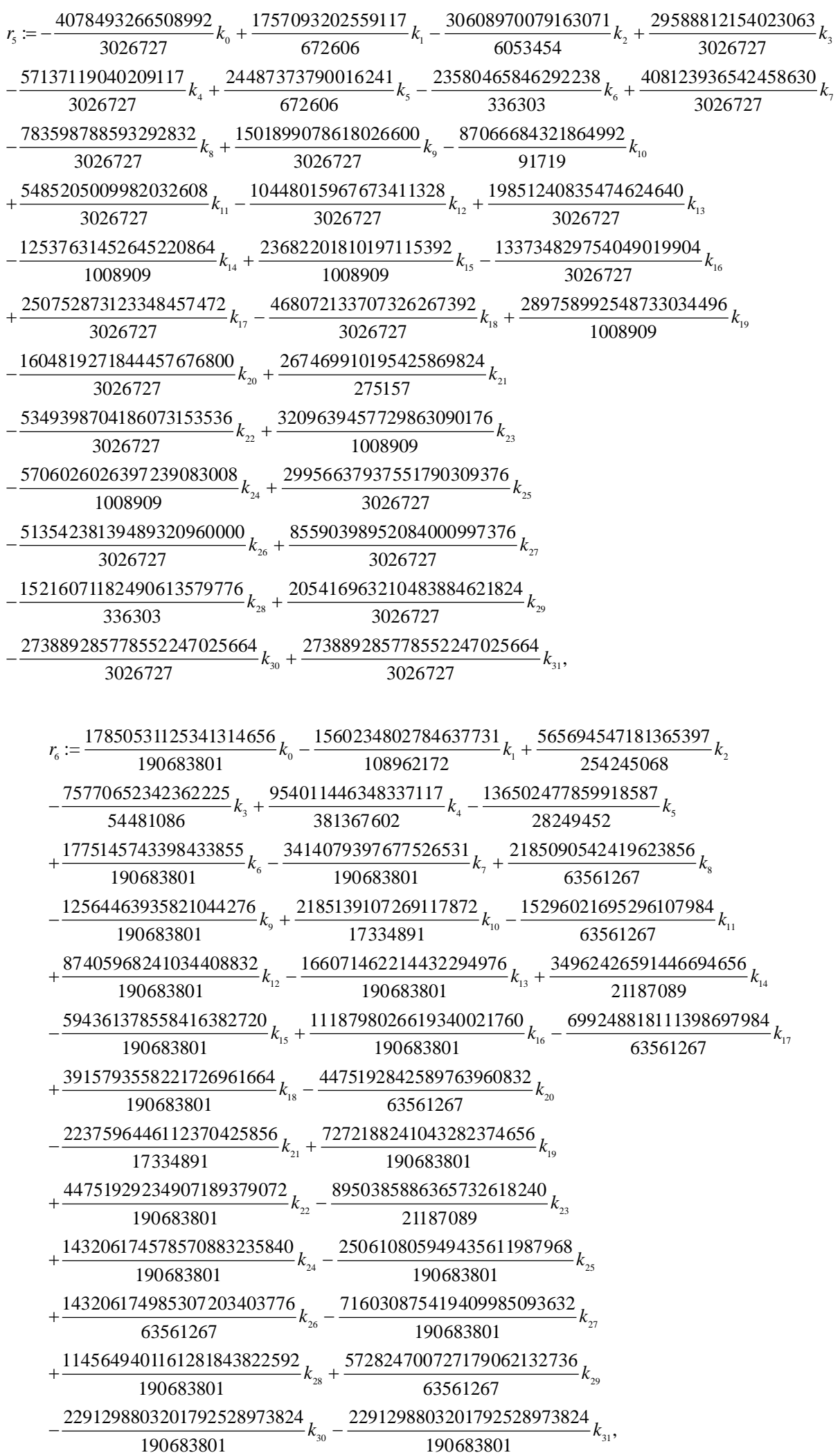




\section{Continued}

$$
\begin{aligned}
& r_{7}:=\frac{182985211619366502400}{27240543} k_{0}-\frac{9114335538551103232}{9080181} k_{1}+\frac{3015119186275104512}{27240543} k_{2} \\
& -\frac{160577127932952064}{27240543} k_{3}-\frac{20805865009788416}{27240543} k_{4}+\frac{593806988273408}{3026727} k_{5} \\
& +\frac{2355269316932608}{9080181} k_{6}-\frac{22249981901335552}{27240543} k_{7}+\frac{47041546286743552}{27240543} k_{8} \\
& -\frac{92318681559486464}{27240543} k_{9}+\frac{1794795498225664}{275157} k_{10}-\frac{339752396451659776}{27240543} k_{11} \\
& +\frac{647414622429839360}{27240543} k_{12}-\frac{1230220929648558080}{27240543} k_{13}+\frac{777003747655745536}{9080181} k_{14} \\
& -\frac{1467684753032151040}{9080181} k_{15}+\frac{8288118568652177408}{27240543} k_{16}-\frac{15540230489066110976}{27240543} k_{17} \\
& +\frac{29008434308301783040}{27240543} k_{18}-\frac{17957602863013756928}{9080181} k_{19} \\
& +\frac{99457493780231684096}{27240543} k_{20}-\frac{16576249008379461632}{2476413} k_{21} \\
& +\frac{331524980412700229632}{27240543} k_{22}-\frac{198914988287781830656}{9080181} k_{23} \\
& +\frac{117875548621602881536}{3026727} k_{24}-\frac{1856539890819019767808}{27240543} k_{25} \\
& +\frac{3182639812846948777984}{27240543} k_{26}-\frac{5304399688084778319872}{27240543} k_{27} \\
& +\frac{2829013166979691249664}{9080181} k_{28}-\frac{16974079001880596381696}{27240543} k_{30} \\
& -\frac{16974079001880596381696}{27240543} k_{31}+\frac{12730559251409835065344}{27240543} k_{29} \text {, } \\
& r_{8}:=\frac{1402046646636032}{572051403} k_{0}-\frac{404359709107292}{81721629} k_{1}+\frac{1838528360224516}{190683801} k_{2} \\
& -\frac{1526510201939944}{81721629} k_{3}+\frac{20651859976599112}{572051403} k_{4}-\frac{1475682550603964}{21187089} k_{5} \\
& +\frac{76747948993198640}{572051403} k_{6}-\frac{147602599162168688}{572051403} k_{7}+\frac{94468543272975104}{190683801} k_{8} \\
& -\frac{543201327526722112}{572051403} k_{9}+\frac{94470348795976448}{52004673} k_{10}-\frac{661294196377898240}{190683801} k_{11} \\
& +\frac{3778828733290707968}{572051403} k_{12}-\frac{7179779137236426752}{572051403} k_{13}+\frac{1511532964276548608}{63561267} k_{14} \\
& -\frac{25696065395082001408}{572051403} k_{15}+\frac{48369069929078318080}{572051403} k_{16}-\frac{30230670898864546816}{190683801} k_{17} \\
& +\frac{193476311878459657216}{190683801} k_{20}+\frac{169291764793840182272}{572051403} k_{18} \\
& -\frac{314399000941203786752}{572051403} k_{19}-\frac{96738157089236403200}{52004673} k_{21} \\
& +\frac{1934763156414028899328}{572051403} k_{22}-\frac{386952633138707606528}{63561267} k_{23} \\
& +\frac{6191242149010792604672}{572051403} k_{24}-\frac{10834673781543178923008}{572051403} k_{25} \\
& +\frac{6191242168369447930880}{190683801} k_{26}-\frac{30956210865390042385408}{572051403} k_{27} \\
& +\frac{49529937408072029873152}{572051403} k_{28}-\frac{24764968711051435418624}{190683801} k_{29} \\
& +\frac{99059874858236582638592}{572051403} k_{30}-\frac{99059874858236582638592}{572051403} k_{31} \text {, }
\end{aligned}
$$




\section{Continued}

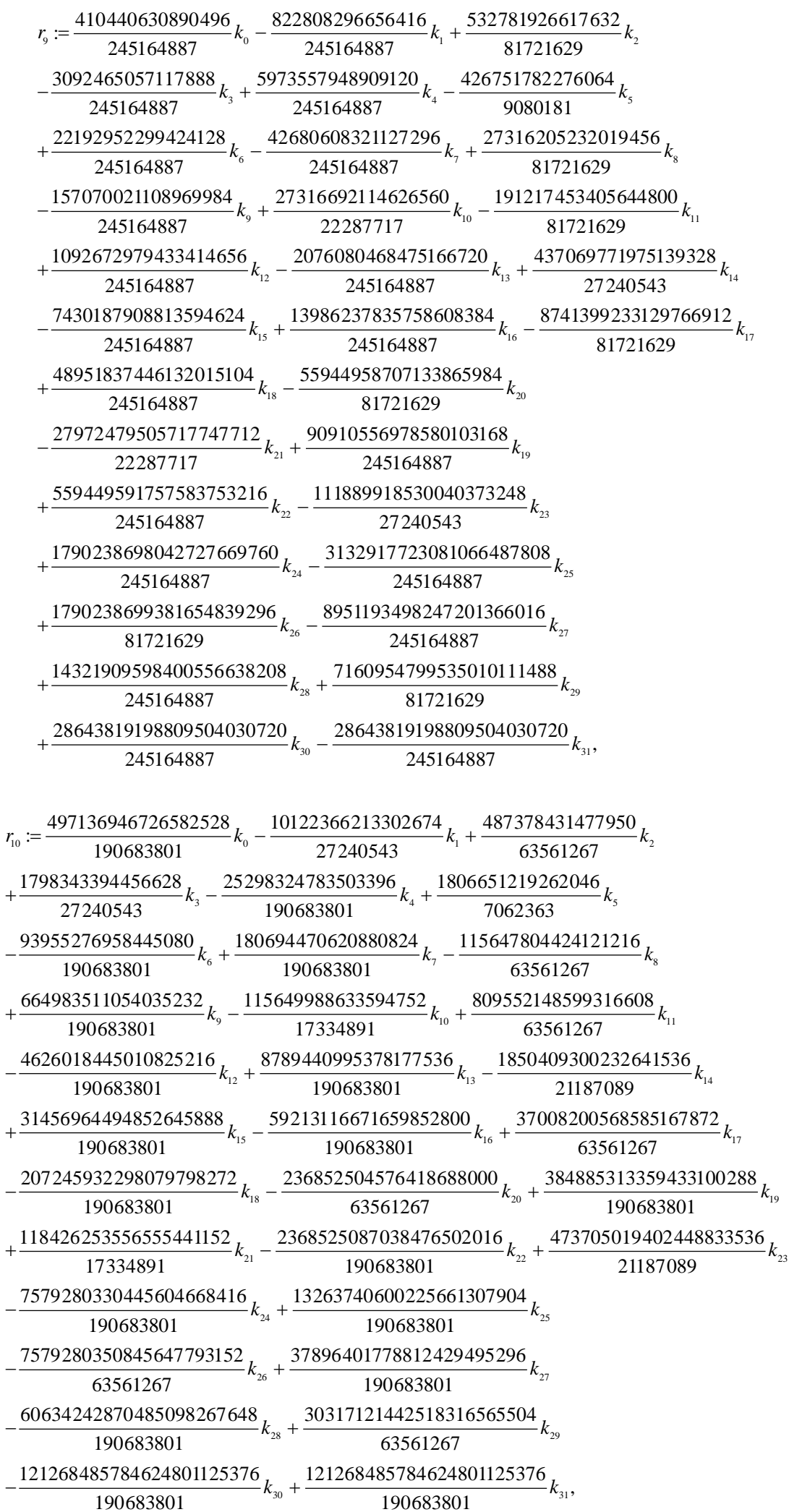




\section{Continued}

$$
\begin{aligned}
& r_{11}:=-\frac{7845155552}{245164887} k_{0}+\frac{1961246513}{980659548} k_{1}+\frac{2735}{326886516} k_{2}-\frac{277}{490329774} k_{3}+\frac{7}{490329774} k_{4}-\frac{1}{36320724} k_{5} \\
& +\frac{13}{245164887} k_{6}-\frac{25}{245164887} k_{7}+\frac{16}{81721629} k_{8}-\frac{92}{245164887} k_{9}+\frac{16}{22287717} k_{10}-\frac{112}{81721629} k_{11}+\frac{640}{245164887} k_{12} \\
& -\frac{1216}{245164887} k_{13}+\frac{256}{27240543} k_{14}-\frac{4352}{245164887} k_{15}+\frac{8192}{245164887} k_{16}-\frac{5120}{81721629} k_{17}+\frac{28672}{245164887} k_{18}-\frac{32768}{81721629} k_{20} \\
& -\frac{16384}{22287717} k_{21}+\frac{327680}{245164887} k_{22}-\frac{65536}{27240543} k_{23}+\frac{53248}{245164887} k_{19}+\frac{1048576}{245164887} k_{24}-\frac{1835008}{245164887} k_{25}+\frac{1048576}{81721629} k_{26} \\
& -\frac{5242880}{245164887} k_{27}+\frac{8388608}{245164887} k_{28}-\frac{16777216}{245164887} k_{30}-\frac{16777216}{245164887} k_{31}+\frac{4194304}{81721629} k_{29}, \\
& r_{12}:=\frac{2317220714287552}{190683801} k_{0}+\frac{152166700793161}{54481086} k_{1}-\frac{1195931905993439}{127122534} k_{2} \\
& +\frac{508464732469555}{27240543} k_{3}-\frac{6883955676246967}{190683801} k_{4}+\frac{983776951113553}{14124726} k_{5} \\
& -\frac{25582341097777850}{190683801} k_{6}+\frac{49200272990040146}{190683801} k_{7}-\frac{31489134650021408}{63561267} k_{8} \\
& +\frac{181064925473328184}{190683801} k_{9}-\frac{31489736491021856}{17334891} k_{10}+\frac{220428740372507360}{63561267} k_{11} \\
& -\frac{1259594386791424256}{190683801} k_{12}+\frac{2393230849565066624}{190683801} k_{13}-\frac{503838245036658176}{21187089} k_{14} \\
& +\frac{8565251833083414016}{190683801} k_{15}-\frac{16122828865249315840}{190683801} k_{16}+\frac{10076768771844355072}{63561267} k_{17} \\
& -\frac{56429907709061295104}{190683801} k_{18}+\frac{32245663474191887360}{17334891} k_{21}-\frac{64491326181712724992}{63561267} k_{20} \\
& +\frac{104798403091518792704}{190683801} k_{19}-\frac{644913274360271358976}{190683801} k_{22}+\frac{128982655490688214016}{21187089} k_{23} \\
& -\frac{2063722494114835057664}{190683801} k_{24}+\frac{3611514371625725305856}{190683801} k_{25} \\
& -\frac{2063722500567720166400}{63561267} k_{26}+\frac{10318612510686201742336}{190683801} k_{27} \\
& -\frac{16509780024913910139904}{190683801} k_{28}+\frac{8254890014795428563968}{63561267} k_{29} \\
& -\frac{33019560063858661243904}{190683801} k_{30}+\frac{33019560063858661243904}{190683801} k_{31}, \\
& r_{13}:=\frac{1713565605536}{3026727} k_{0}-\frac{4885835317153}{4035636} k_{1}+\frac{28580433958345}{12106908} k_{2}-\frac{27640118395825}{6053454} k_{3}+\frac{53375164694971}{6053454} k_{4} \\
& -\frac{22875194940935}{1345212} k_{5}+\frac{33042120966595}{1008909} k_{6}-\frac{190628577556325}{3026727} k_{7}+\frac{366008629043312}{3026727} k_{8}-\frac{701519765914156}{3026727} k_{9} \\
& +\frac{40667991081200}{91719} k_{10}-\frac{2562094151981840}{3026727} k_{11}+\frac{4880198724104320}{3026727} k_{12}-\frac{9272412472391104}{3026727} k_{13} \\
& +\frac{5856281324390144}{1008909} k_{14}-\frac{3687300576400640}{336303} k_{15}+\frac{62467405675601920}{3026727} k_{16}-\frac{117126728485473280}{3026727} k_{17} \\
& +\frac{218637154101997568}{3026727} k_{18}-\frac{15038572140081152}{112101} k_{19}+\frac{749616704535101440}{3026727} k_{20}-\frac{124936378636779520}{275157} k_{21} \\
& +\frac{2498732274592317440}{3026727} k_{22}-\frac{1499241872412311552}{1008909} k_{23}+\frac{2665322785088208896}{1008909} k_{24}-\frac{13992962175311544320}{3026727} k_{25} \\
& +\frac{23987960234246144000}{3026727} k_{26}-\frac{39979967159169187840}{3026727} k_{27}+\frac{21322662525727145984}{1008909} k_{28} \\
& +\frac{95952021488282894336}{3026727} k_{29}-\frac{127936055399384350720}{3026727} k_{30}-\frac{127936055399384350720}{3026727} k_{31} \text {, }
\end{aligned}
$$




\section{Continued}

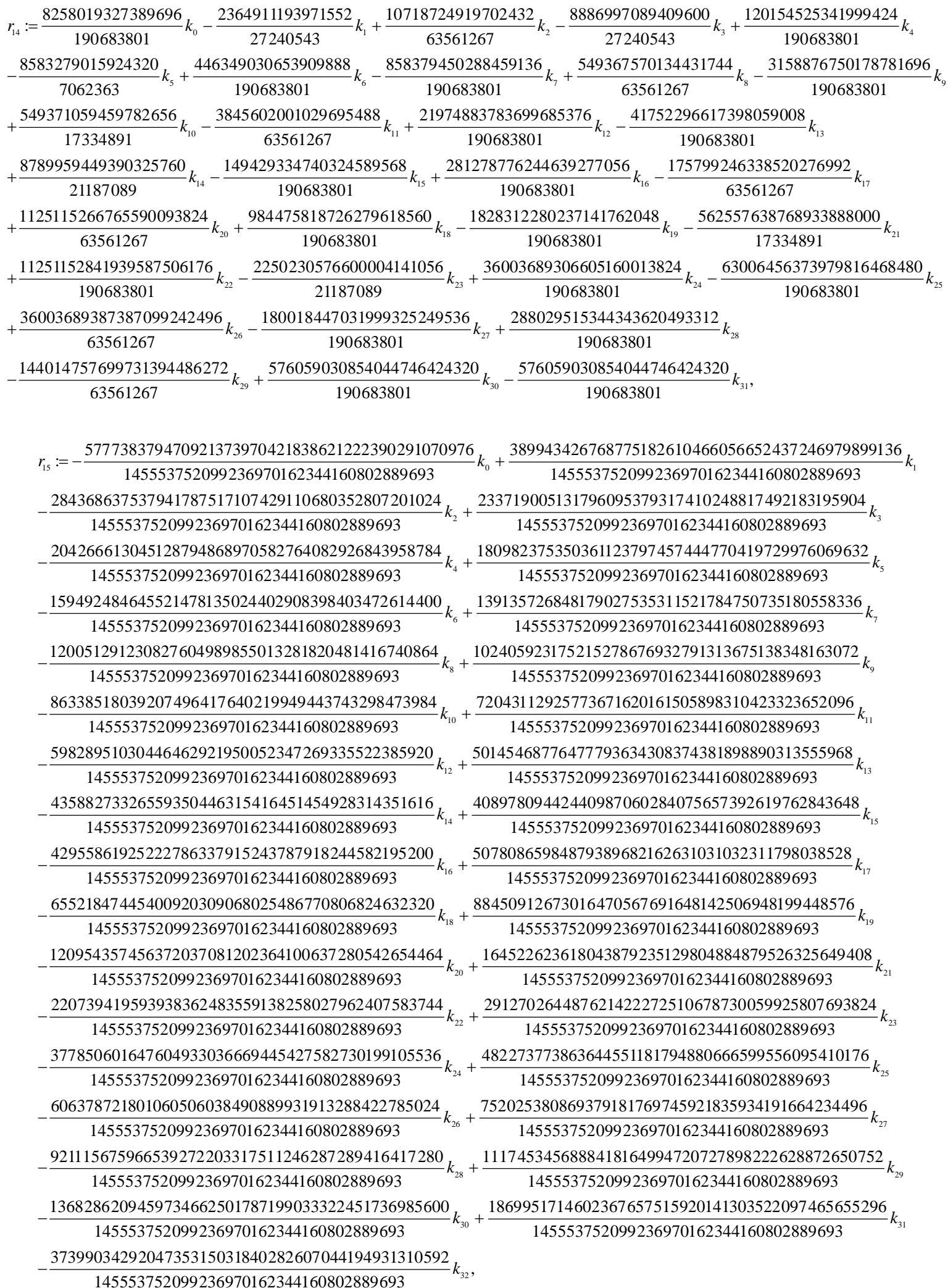




\section{Continued}

$r_{16}:=-\frac{8187517611412321816450317517847180936670908416}{1892198777290080612110474090437566009} k_{0}-\frac{159533088530622432903456266215894185920157280}{1892198777290080612110474090437566009} k_{1}$

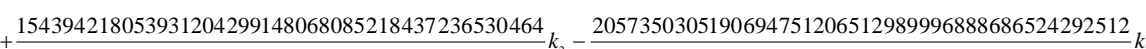

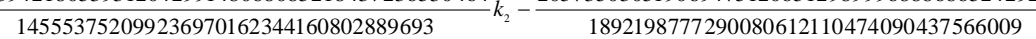

$1732404887835464639120997787916801947574461376 k_{4}$ 1892198777290080612110474090437566009 $-\frac{1405990680809729370498243610916705554858690240}{1892198777290080612110474090437566009} k_{5}$ $+\frac{1144488651965894568749363790437907726384136832}{1892198777290080612110474090437566009} k_{6}$

939844587890153231821926456516923457051665920 1892198777290080612110474090437566009

$+\frac{775530716458450573108031044536332857186887680}{1892198777290080612110474090437566009} k_{8}$

$-\frac{641215590606143303960670664662711172566602752}{1} k$ 1892198777290080612110474090437566009 $+\frac{533598746312304368323837358420614698018730496}{1892198777290080612110474090437566009} k_{10}$

$-\frac{34978573114728227134846608592592432234501632}{145553752099236970162344160802889693} k_{11}$ 145553752099236970162344160802889693 $+\frac{410580247182314008263876827317175039683871744}{1892198777290080612110474090437566009} k_{12}$ $-\frac{410429903343374999751381395132964252726148096}{1892198777290080612110474090437566009} k_{13}$ $+\frac{466571397956138819403384731590732091436726272}{1892198777290080612110474090437566009} k_{14}$

$-\frac{594395101150790885129246689338994483319664640}{15}$ 1892198777290080612110474090437566009

$+\frac{812520028837847841388581651545269952176578560}{1892198777290080612110474090437566009} k_{16}$ 1892198777290080612110474090437566009

$-\frac{1142939484077410993309324621624796185836421120}{18921987772900080612110474090437566009} k_{17}$ 1892198777290080612110474090437566009

$+\frac{1611122320404854212801419430219237356852150272}{1892198777290080612110474090437566009} k_{18}$ 1892198777290080612110474090437566009

$-\frac{2246042283397163284978426120120548188157902848}{19} k_{19}$ 1892198777290080612110474090437566009 $+\frac{3080125446429399549359394621911824478685888512}{1892198777290080612110474090437566009} k_{20}$ $-\frac{4149118181721195882382697028838574923427086336}{1892198777290080612110474090437566009} k_{21}$ $+\frac{422452804013804374185584906133418118056509440}{145553752099236970162344160802889693} k_{22}$

$-\frac{7150157392461355980247977668273897149643358208}{18921987772900080612110474090437566009} k_{23}$ 1892198777290080612110474090437566009 $+\frac{9168214513517564713799636815721714863928508416}{1892198777290080612110474090437566009} k_{24}$ $-\frac{11592569199135583258269488881518337074348949504}{1892198777290080612110474090437566009} k_{25}$ $+\frac{14471584786483274457333207749537373737259892736}{1892198777290080612110474090437566009} k_{26}$

$-\frac{17854724727195931687505586900600532885838233600}{1892198777290080612110474090437566009} k_{27}$ 1892198777290080612110474090437566009 $+\frac{21793652701843709390979659009425718706904236032}{1892198777290080612110474090437566009} k_{28}$ $-\frac{26391696071566916452141489425448739574098427904}{1892198777290080612110474090437566009} k_{29}$

$+\frac{32307970231440982228678836455867430533548998656}{18921987772900080612110474090437566009} k_{30}$ 1892198777290080612110474090437566009

$44140518551189113781753530516704812452450140160 k$ 1892198777290080612110474090437566009

$+\frac{88281037102378227563507061033409624904900280320}{1892198777290080612110474090437566009} k_{32}$, 


\section{Continued}

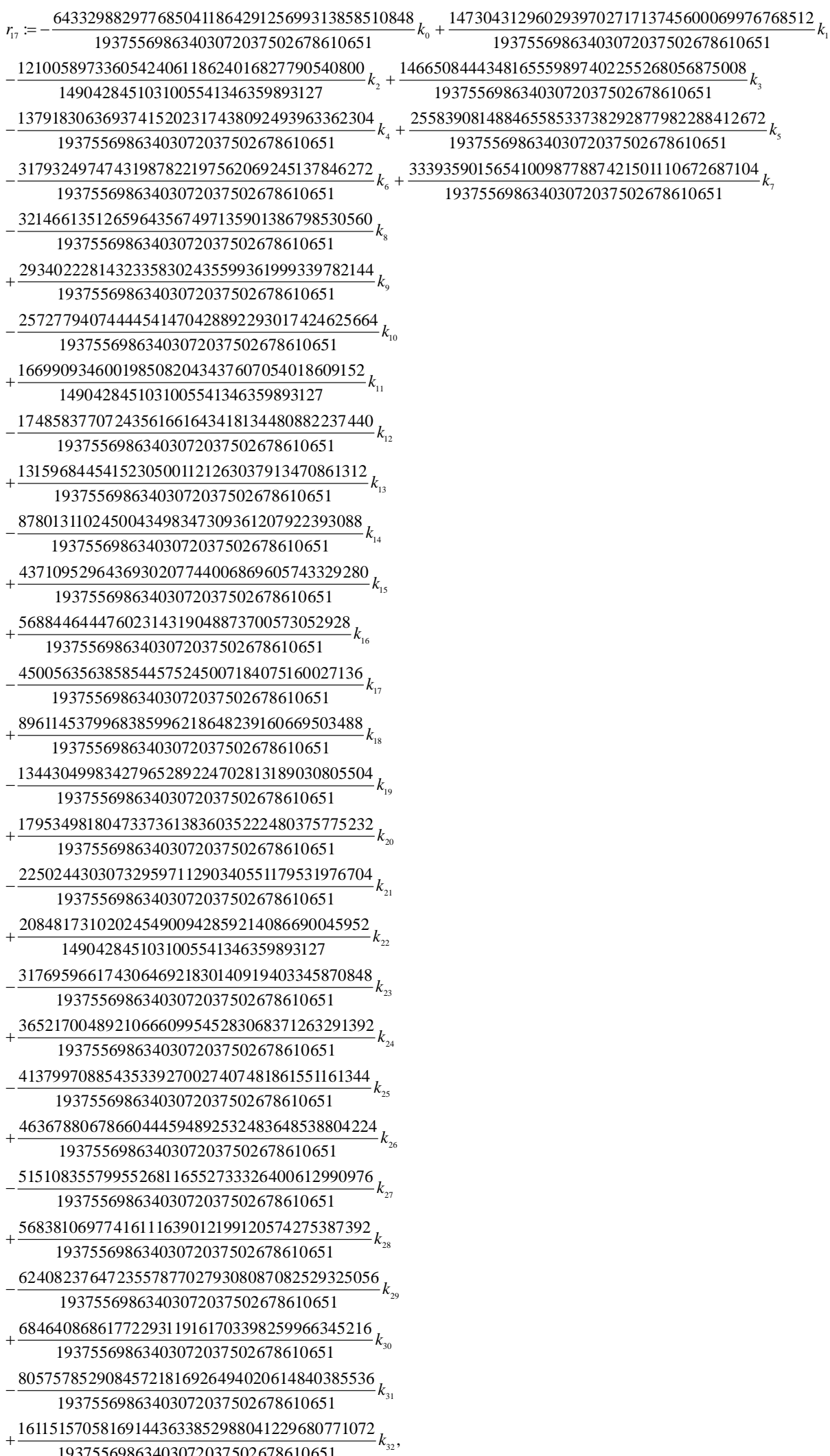




\section{Continued}

$r_{18}:=\frac{7200817595893180445536096846534860064747520}{210244308587786734678941565604174001} k_{0}-\frac{6998804769585734064045981619545288517039456}{210244308587786734678941565604174001} k_{1}$

$+\frac{530440027226536076672531909649765764319776}{16172639122137441129149351200321077} k_{2}-\frac{6824456919060819825768983080500742798450336}{210244308587786734678941565604174001} k_{3}$ $16172639122137441129149351200321077 \quad k_{2}-\frac{210244308587786734678941565604174001}{6}$

$+\frac{6745344487584820041562803126194655389563328}{210244308587786734678941565604174001} k_{4}-\frac{6631258703671288380471438380101647464928448}{210244308587786734678941565604174001} k_{5}$

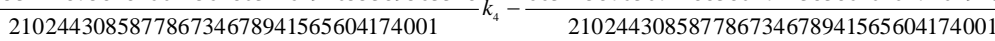

$+\frac{6455113775897888780618057957975709314213504}{210244308587786734678941565604174001} k_{6}$

210244308587786734678941565604174001

$-\frac{6181235253021515552883083212777760046814720}{k_{7}}$ 210244308587786734678941565604174001

$+\frac{5758119554980403429819093457588757301356544}{210244308587786734678941565604174001} k_{8}$ 210244308587786734678941565604174001

$-\frac{5110522418215584299116111920300401752339456}{2} k_{9}$ 210244308587786734678941565604174001 $+\frac{4129576966405346966954698319541915127431680}{210244308587786734678941565604174001} k_{10}$ $-\frac{204626082710675433817370075612361538504192}{16172639122137441129149351200321077} k_{11}$

$+\frac{484884300610749283881335487138637146285056}{210244308587786734678941565604174001} k_{12}$

$+\frac{2694985478451938699706852245592271879365632}{210244308587786734678941565604174001} k_{13}$

$-\frac{7282123882134533727480605253259587403565056}{210244308587786734678941565604174001} k_{14}$ 210244308587786734678941565604174001

$+\frac{13807628039746943061694296140356476279492608}{210244308587786734678941565604174001} k_{15}$ 210244308587786734678941565604174001 $-\frac{22956294155949443695821205372887579311153152}{210244308587786734678941565604174001} k_{16}$ $+\frac{35590428396275037094309238771169584563453952}{210244308587786734678941565604174001} k_{17}$

$-\frac{52769844592919353622636487797537224668282880}{210244308587786734678941565604174001} k_{\text {вв }}$ 210244308587786734678941565604174001

$+\frac{75765966797134442187626316939977444164370432}{210244308587786734678941565604174001} k_{19}$ 210244308587786734678941565604174001 $-\frac{106068830099335348925610826044828633042780160}{210244308587786734678941565604174001} k_{20}$

$+\frac{145386924761036033230957881256012322449915904}{210244308587786734678941565604174001} k_{21}$ 21024430858778673467894156560417400

$15049279328586904693634985775596926369529856 k_{22}$ 16172639122137441129149351200321077 $+\frac{258950098403709600992922925102622957931855872}{210244308587786734678941565604174001} k_{23}$ $-\frac{337618625261985621511998958776201715539509248}{210244308587786734678941565604174001} k_{24}$

$+\frac{434113669315911983121555959326471394635022336}{25} k_{25}$ 210244308587786734678941565604174001

$-\frac{551043106399733822502402833747296646861946880}{2} k_{26}$ 210244308587786734678941565604174001

$+\frac{691107750712581020350483488820461979164999680}{210244308587786734678941565604174001} k_{2}$ 210244308587786734678941565604174001

$857194293183352945392011830903591609091227648 k_{28}$ 210244308587786734678941565604174001

$+\frac{1055076115669848335079415672711933971734724608}{210244308587786734678941565604174001} k_{29}$ $-\frac{1316548498187790654058570513970701799812759552}{210244308587786734678941565604174001} k_{30}$

$+\frac{1839493263223675292016880196488237455968829440}{210244308587786734678941565604174001} k_{31}$ 210244308587786734678941565604174001

$-\frac{3678986526447350584033760392976474911937658880}{210244308587786734678941565604174001} k_{32}$, 


\section{Continued}

$r_{19}:=-\frac{846331556576961593459224129208633418441519104}{1892198777290080612110474090437566009} k_{0}+\frac{376685845259126425679159929193982114613026400}{1892198777290080612110474090437566009} k_{1}$

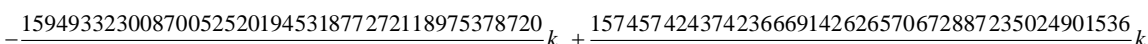
$145553752099236970162344160802889693 \quad k_{2}+\frac{1892198777290080612110474090437566009}{1340524065174728537302909821900119102070678}$ $-\frac{142757330201442573398181736488684165478953920}{1892198777290080612110474090437566009} k_{4}+\frac{134052406517472853730290982190011910207067840}{1892198777290080612110474090437566009} k_{5}$

$-\frac{124259776276963153692996588503959035499995776}{1892198777290080612110474090437566009} k_{0}$ 1892198777290080612110474090437566009 $+\frac{112921269720863783677292219739652208623232512}{1892198777290080612110474090437566009} k_{7}$ $-\frac{100743677662559060822473284906275626382305280}{1892198777290080612110474090437566009} k_{\text {в }}$

$+\frac{88244241456936317146220738290478244155806720}{1892198777290080612110474090437566009} k_{9}$

$-\frac{75651309378062869097629433955447348840395264}{1892198777} k_{1}$ 1892198777290080612110474090437566009

$+\frac{4849031507067062092856558657652005348873728}{1455553752099236970162344160802889693} k_{11}$ 145553752099236970162344160802889693

- $\frac{50417462117028014651397187179764440190501888}{18921987772900080612110474090437566009} k_{12}$ 1892198777290080612110474090437566009

$+\frac{37790993654245889191778157000988234520083456}{1892198777290080612110474090437566009} k_{13}$ 1892198777290080612110474090437566009

$-\frac{25154311114530973778498491223713198830811136}{1892198777290080612110474090437566009} k_{14}$ 1892198777290080612110474090437566009

$+\frac{12501854301022655068735728536552256371814400}{1892198777290080612110474090437566009} k_{15}$

74587763380230960804941100576736416768000 1892198777290080612110474090437566009

12886880971902362237482557821386000286842880 1892198777290080612110474090437566009

$+\frac{25651762948709973544400826181308544276037632}{18921987772900080612110474090437566009} k_{18}$ 1892198777290080612110 $-\frac{38492215473544975648972869943687091588956160}{1892198777290080612110474090437566009} k_{19}$

. $+\frac{51438870966597824196638913979421144158044160}{1892198777290080612110474090437566009} k_{20}$ 1892198777290080612110474090437566009

$-\frac{64531272182658442150283331484088449896808448}{1892198777290080612110474090437566009} k$ 1892198777290080612110474090437566009

$+\frac{5986060837788585057029513883773431389356032}{145553752099236970162344160802889693} k_{2}$ 145553752099236970162344160802889693

_ $91361089805231262070827247863264282107445248 k$ 1892198777290080612110474090437566009 $+\frac{105228168991844362841136407442766957360709632}{1892198777290080612110474090437566009} k_{24}$

$-\frac{119500079234285383183585405562083118156349440}{18} k_{25}$ 1892198777290080612110474090437566009

$+\frac{134265969294232210675100001389251263902777344}{1892198777290080612110474090437566009} k_{26}$ 189219877729008061211047409043756600

$-\frac{149622180436917923372056747595921941211906048}{k} k$ 1892198777290080612110474090437566009 $+\frac{165679438934685980289733783860969791102648320}{1892198777290080612110474090437566009} k_{28}$ $-\frac{182659197332223299458687389542144095612829696}{1892198777290080612110474090437566009} k_{29}$

$+\frac{201483955529299143130194134055571309361889280}{18921987772900080612110474090437566009} k_{30}$ 1892198777290080612110474090437566009

$-\frac{239133471923450830473207623082425736860008448}{1892198777290080612110474090437566009} k_{3}$ 1892198777290080612110474090437566009

$+\frac{478266943846901660946415246164851473720016896}{1892198777290080612110474090437566009} k_{32}$ 1892198777290080612110474090437566009 


\section{Continued}

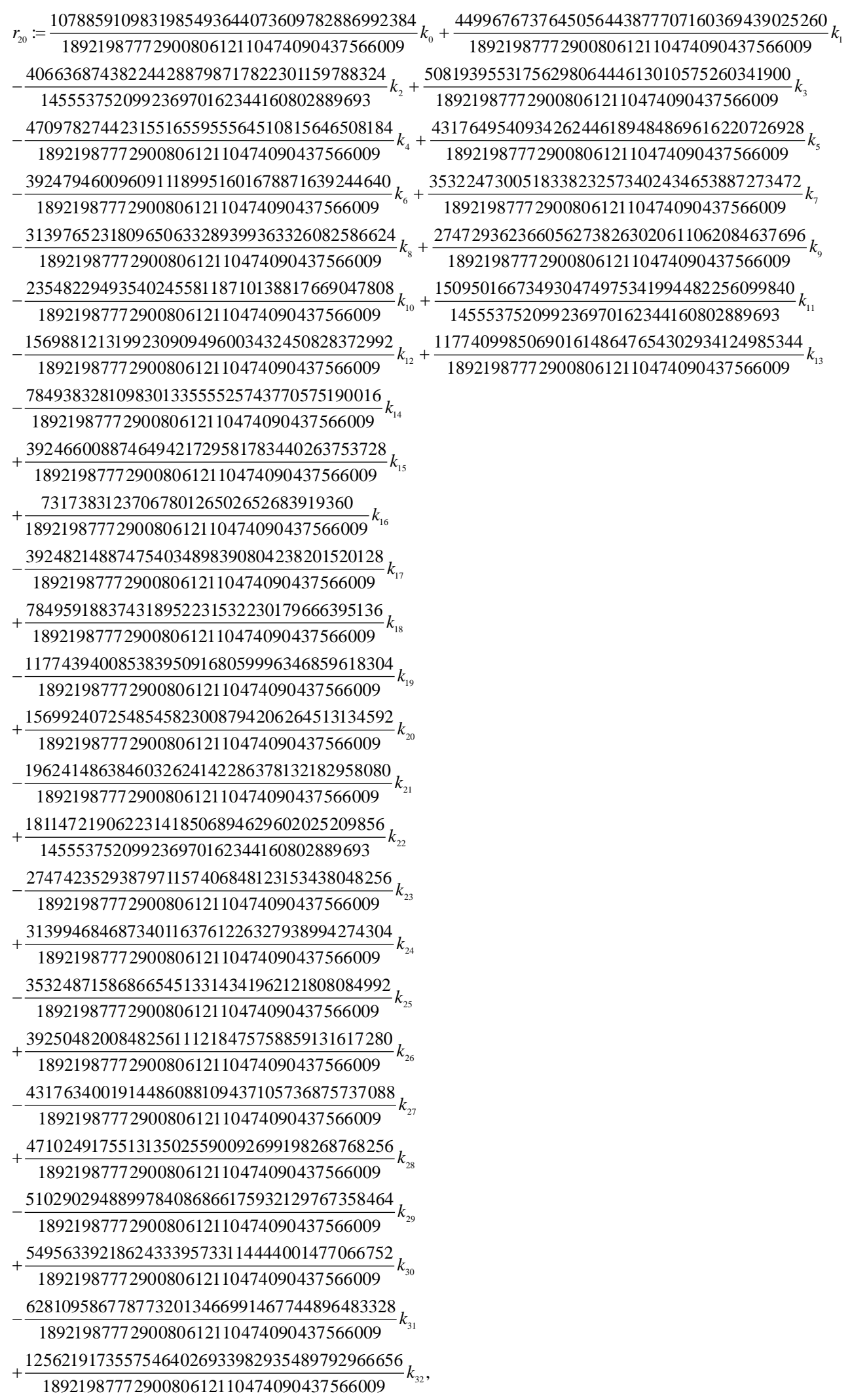




\section{Continued}

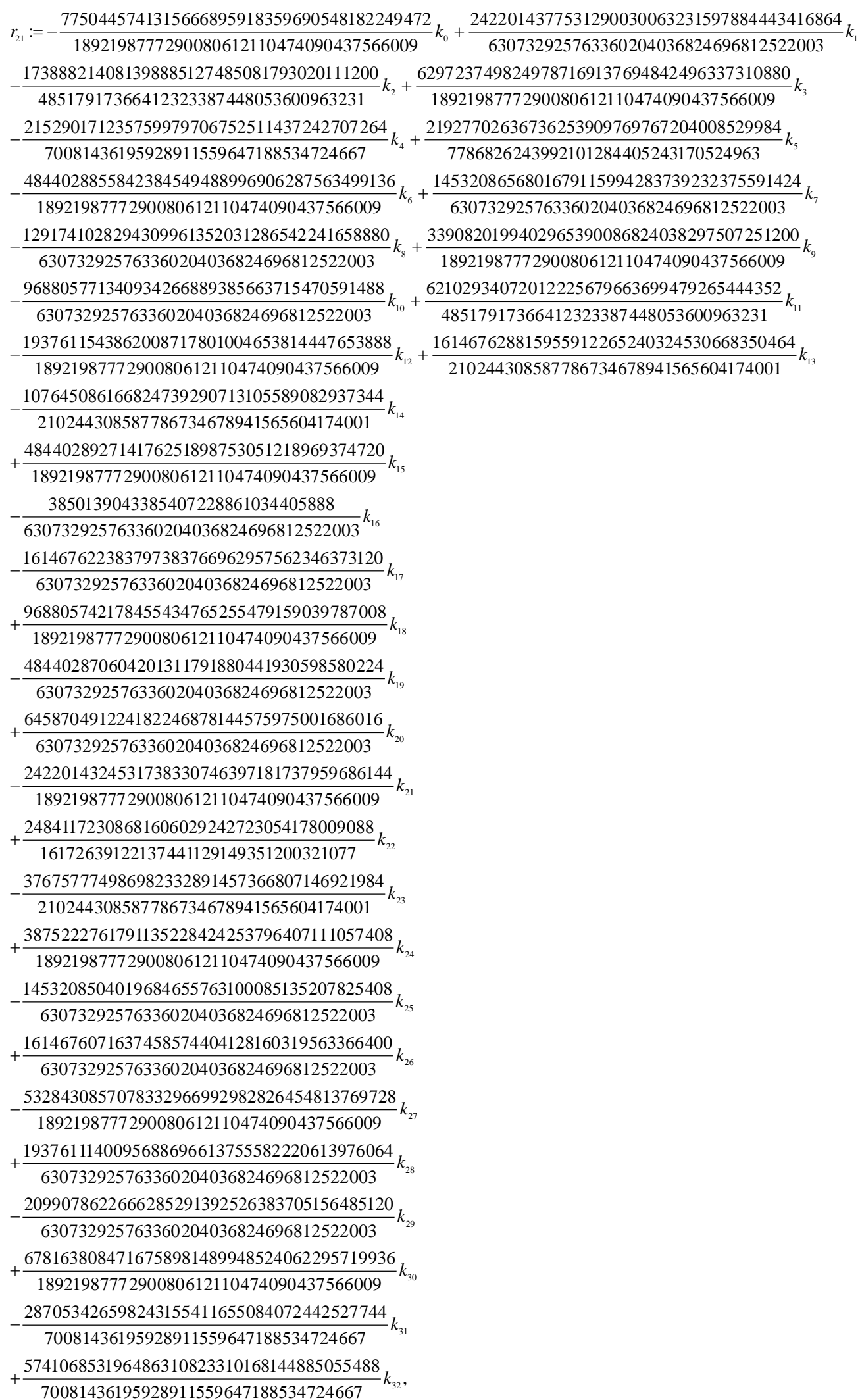




\section{Continued}

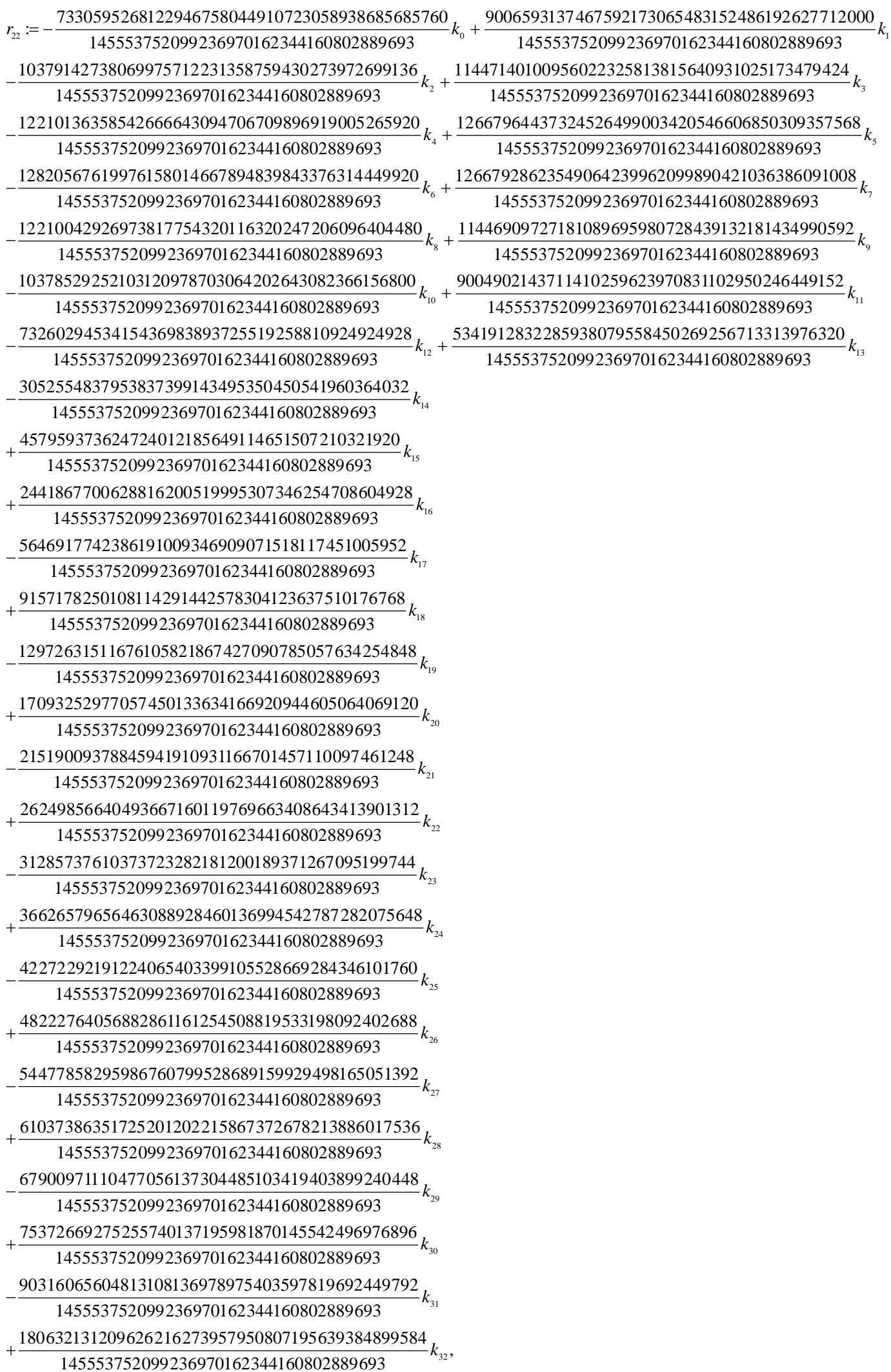




\section{Continued}

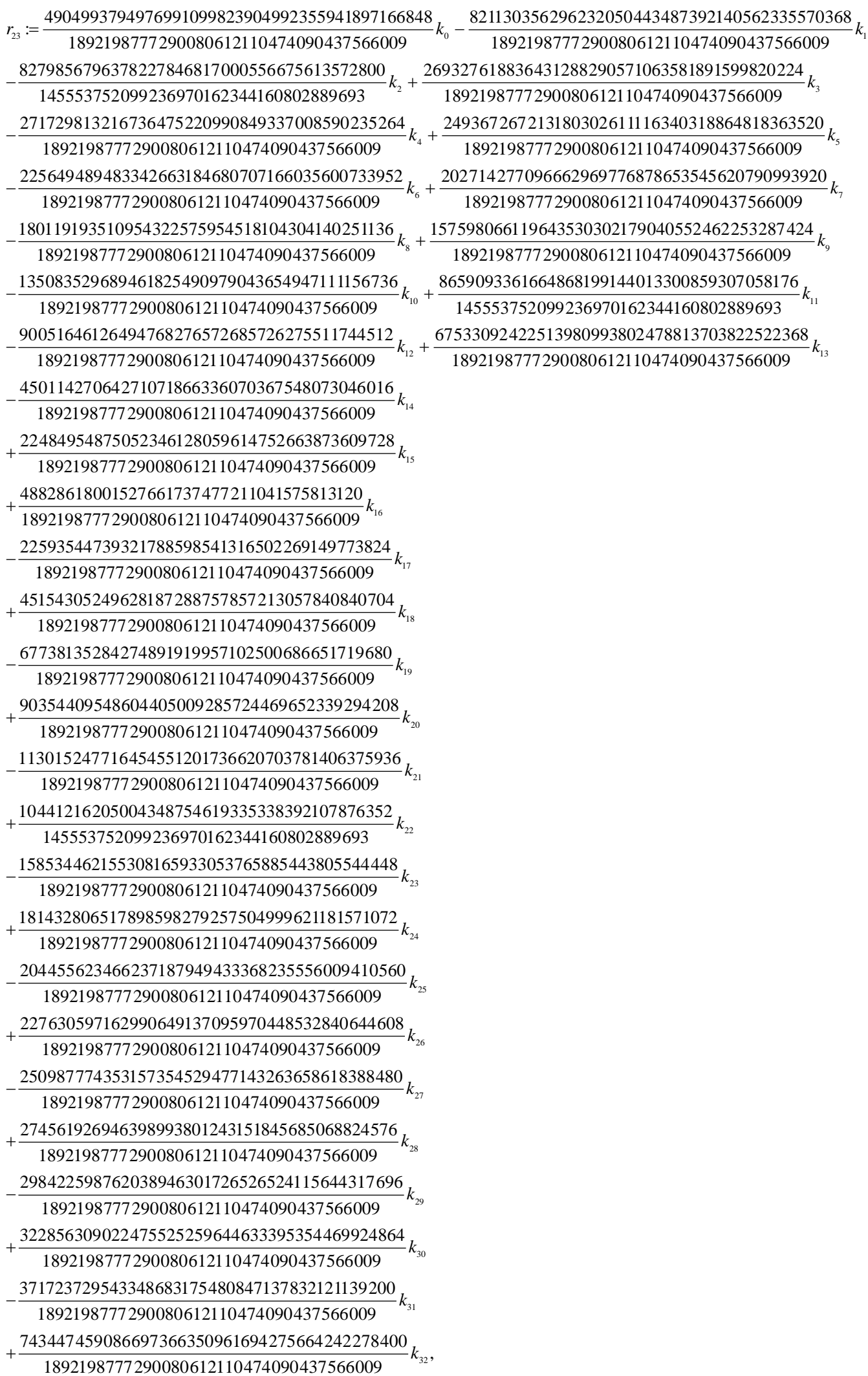




\section{Continued}

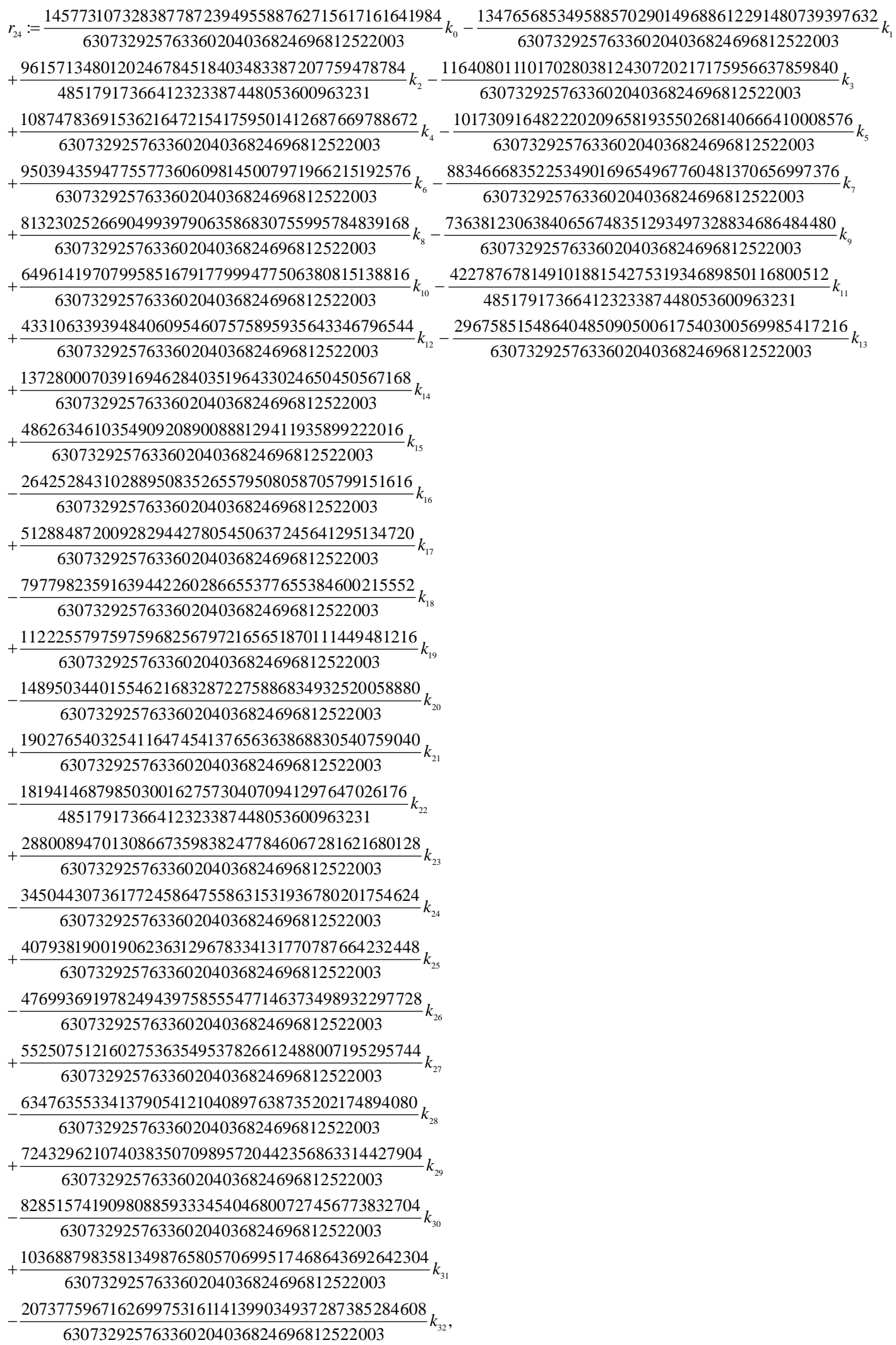




\section{Continued}

$r_{25}:=\frac{30369535433400425692686935693374976}{523140386311882945012572322487577} k_{0}-\frac{30548727162750800714204873557216492}{523140386311882945012572322487577} k$

$+\frac{2341156218945525925121338143139748}{40241568177837149616351717114429} k_{2}-\frac{30024872588068556611637930492565772}{523140386311882945012572322487577} k_{3}$

$+\frac{29232599827905537079386264036365336}{523140386311882945012572322487577} k_{4}-\frac{27842804442099501942911262650939152}{523140386311882945012572322487577} k_{5}$

$+\frac{25459204842448720792168607543575520}{523140386311882945012572322487577} k_{6}-\frac{21407452619120992442617832457534592}{523140386311882945012572322487577} k_{7}$

$+\frac{14567206920061526753831976512856320}{523140386311882945012572322487577} k_{8}-\frac{3099338359407216404603652645626368}{523140386311882945012572322487577} k_{9}$

$-\frac{15984819201215476418236924769695744}{523140386311882945012572322487577} k_{10}+\frac{3653167927184696226890820477117440}{40241568177837149616351717114429} k_{11}$

$-\frac{99060197958369474007053504906299392}{523140386311882945012572322487577} k_{12}+\frac{182687880879359203066337060250460160}{523140386311882945012572322487577} k_{13}$

$-\frac{316952615901078579346220447703138304}{523140386311882945012572322487577} k_{14}$

$+\frac{530197867579982217365618357622456320}{523140386311882945012572322487577} k_{15}$

$-\frac{864958160190631396682799005983473664}{523140386311882945012572322487577} k_{16}$

$+\frac{1383923756297083969736486490216366080}{523140386311882945012572322487577} k_{17}$ $-\frac{2177695291120799781815050956642254848}{523140386311882945012572322487577} k_{18}$

$+\frac{3374461682473263310402763715600416768}{523140386311882945012572322487577} k_{19}$

$-\frac{5151536628577199013849272625507991552}{523140386311882945012572322487577} k_{20}$

$+\frac{7748434988578592750116470679442849792}{523140386311882945012572322487577} k_{21}$

$-\frac{883146802636934355272972131593551872}{40241568177837149616351717114429} k_{22}$ 40241568177837149616351717114429

$+\frac{16755082077855360812570820857829883904}{523140386311882945012572322487577} k_{23}$ $-\frac{24080278412759568541138590877071179776}{5} k$ 523140386311882945012572322487577

$+\frac{34077701242270576134585448479248777216}{523140386311882945012572322487577} k_{25}$

$47480839583288513519265530714712506368 k_{26}$ 523140386311882945012572322487577

$+\frac{65136275473935535166839023743392514048}{523140386311882945012572322487577} k_{27}$ 88116776994777844639578211944379580416 $523140386311882945012572322487577 k_{28}$ $+\frac{118567880306947849062027664075855265792}{523140386311882945012572322487577} k_{29}$ $163960187201773243383897644068308189184 k_{30}$ 52314038631188294501257232248757

$+\frac{254744800991424032027637604053214035968}{} k_{3}$ 523140386311882945012572322487577

$-\frac{509489601982848064055275208106428071936}{523140386311882945012572322487577} k_{32}$, 


\section{Continued}

$r_{26}:=-\frac{532576647580652673203087099587540161536}{630732925763360204036824696812522003} k_{0}-\frac{17892618423041325884716514266653926008}{7786826243992101284405243170524963} k_{1}$ $+\frac{44505801013510081263317990655872221688}{16172639122137441129149351200321077} k_{2}-\frac{1672658978849833322237280836145112668200}{630732925763360204036824696812522003} k_{3}$ $+\frac{516858628044770681060100011005773225232}{210244308587786734678941565604174001} k_{4}-\frac{473827854412874945611124169162681013808}{210244308587786734678941565604174001} k_{5}$ $+\frac{1292138011589718117529956743103176106592}{630732925763360204036824696812522003} k_{6}-\frac{387633468742758261653150900325437399296}{210244308587786734678941565604174001} k_{7}$ $+\frac{114853965527070992712320085170737263616}{70081436195928911559647188534724667} k_{8}-\frac{904474643717864850160291642596684798464}{630732925763360204036824696812522003} k_{\mathrm{g}}$ $+\frac{86140436151357529754116173259486728448}{70081436195928911559647188534724667} k_{10}-\frac{16565465879121948241030372065412737280}{16172639122137441129149351200321077} k_{11}$ $+\frac{516842352657515900808283672661766988288}{630732925763360204036824696812522003} k_{12}-\frac{129210479985959956144078257625580354048}{210244308587786734678941565604174001} k_{13}$ $+\frac{86140125592125736075381144567574115328}{210244308587786734678941565604174001} k_{14}$ $-\frac{129209081193113319790421238556112648192}{630732925763360204036824696812522003} k_{1}$ 630732925763360204036824696812522003 $-\frac{855922860290834750886914685644800}{210244308587786734678941565604174001} k_{16}$ $+\frac{4785731294025498979112326425977307136}{23360478731976303853215729511574889} k_{17}$ $-\frac{258427696931956483440618642038062481408}{630732925763360204036824696812522003} k_{18}$ $+\frac{43071306930394493399559667223004053504}{70081436195928911559647188534724667} k_{19}$ $-\frac{172285797542331673982701672178729353216}{210244308587786734678941565604174001} k_{20}$ $+\frac{646075170355437997575776455063803658240}{630732925763360204036824696812522003} k_{21}$ $\frac{19879380078007994839052436513703067648}{16172639122137441129149351200321077} k_{22}$ 16172639122137441129149351200321077 $+\frac{301506743245555160679099524221938171904}{210244308587786734678941565604174001} k_{23}$ $-\frac{1033749421566836849380460077081460473856}{630732925763360204036824696812522003} k_{24}$ $+\frac{387661525703268081381862327465684238336}{210244308587786734678941565604174001} k_{25}$ $-\frac{143580778607988633398265251857243832320}{70081436195928911559647188534724667} k_{26}$ $+\frac{1421478126450221242462251569114767163392}{630732925763360204036824696812522003} k_{27}$ $-\frac{57434798267534328613775401875081789440}{23360478731976303853215729511574889} k_{28}$ $+\frac{560004842049606198832984011105131036672}{210244308587786734678941565604174001} k_{29}$ $\frac{1809316591378582508060632018355065192448}{6} k_{30}$ 630732925763360204036824696812522003 $+\frac{689306907279370110394663996144803119104}{210244308587786734678941565604174001} k_{3}$ 1378613814558740220789327992289606238208 21024430858778673467894156560417400 


\section{Continued}

$$
\begin{aligned}
& r_{27}:=-\frac{244068804056062971184384802624086696960}{1892198777290080612110474090437566009} k_{0}+\frac{245521992973420144792619170884545569064}{1892198777290080612110474090437566009} k_{1} \\
& -\frac{18818002780861340372080281508236661624}{145553752099236970162344160802889693} k_{2}+\frac{241386155217670164379934096448071769272}{18921987772290080612110474090437566009} k_{3} \\
& -\frac{235109091584591796519536925401669864464}{1892198777290080612110474090437566009} k_{4}+\frac{224107060741608931164832553996481610160}{1892198777290080612110474090437566009} k_{5} \\
& -\frac{205260277431798626342847967011474345760}{1892198777290080612110474090437566009} k_{6}+\frac{173268106619364970686911686796305998080}{1892198777290080612110474090437566009} k_{7} \\
& -\frac{119343228131200414742433269507040572416}{1892198777290080612110474090437566009} k_{8}+\frac{29097575415579745749550262518087516160}{1892198777290080612110474090437566009} k_{9} \\
& +\frac{120780124052006402899134663315586976000}{1892198777290080612110474090437566009} k_{10}-\frac{28280346398185899917770346094335946496}{145553752099236970162344160802889693} k_{11} \\
& +\frac{770638150237652519851251085624617302528}{1892198777290080612110474090437566009} k_{12}-\frac{1422168184083610334083018337916074206720}{1892198777290080612110474090437566009} k_{13} \\
& +\frac{2464519536666052652128858971369315666944}{1892198777290080612110474090437566009} k_{14}-\frac{4113260258774340391564396738165787318272}{1892198777290080612110474090437566009} k_{15} \\
& +\frac{6689185183531792382450120946849544318976}{1892198777290080612110474090437566009} k_{16}-\frac{10660308483866487668880657477762188591104}{1892198777290080612110474090437566009} k_{17} \\
& +\frac{16694655874267023044770741038570563403776}{1892198777290080612110474090437566009} k_{18}-\frac{25723177991669238020353294889882271219712}{1892198777290080612110474090437566009} k_{19} \\
& +\frac{39010077435528022426519049144709607325696}{1892198777290080612110474090437566009} k_{20}-\frac{58225712707746316710402738568300498911232}{1892198777290080612110474090437566009} k_{21} \\
& +\frac{6578154782971990678785047592218031030272}{145553752099236970162344160802889693} k_{22}-\frac{123562899340355793462446913145903217901568}{1892198777290080612110474090437566009} k_{23} \\
& +\frac{175631366879817503109242552996541448060928}{1892198777290080612110474090437566009} k_{24}-\frac{245594474635033919818807930188334132363264}{1892198777290080612110474090437566009} k_{25} \\
& +\frac{337914195318356698462408678816680277704704}{1892198777290080612110474090437566009} k_{26}-\frac{457603105962950783222262237749319730462720}{1892198777290080612110474090437566009} k_{27} \\
& +\frac{610774992243607696902489655398674015977472}{1892198777290080612110474090437566009} k_{28}-\frac{809657425509910485111333028589586184929280}{1892198777290080612110474090437566009} k_{29} \\
& +\frac{1099960952747505022377408312863614120755200}{1892198777290080612110474090437566009} k_{30}-\frac{1680568007222694096909558881411669992407040}{1892198777290080612110474090437566009} k_{31} \\
& +\frac{3361136014445388193819117762823339984814080}{1892198777290080612110474090437566009} k_{32} \text {. }
\end{aligned}
$$

$$
\eta^{a_{1}}(z) \eta^{a_{2}}(2 z) \eta^{a_{3}}(3 z) \eta^{a_{4}}(4 z) \eta^{a_{6}}(6 z) \eta^{a_{12}}(12 z)=\delta\left(b_{1}\right)+\sum_{n=1}^{\infty} c(n) q^{n},
$$

where for $n \in \mathbb{N}$,

$$
\begin{aligned}
c(n)= & -c_{1} \sigma_{15}(n)-c_{2} \sigma_{15}\left(\frac{n}{2}\right)-c_{3} \sigma_{15}\left(\frac{n}{3}\right)-c_{4} \sigma_{15}\left(\frac{n}{4}\right)-c_{6} \sigma_{15}\left(\frac{n}{6}\right) \\
& -c_{12} \sigma_{15}\left(\frac{n}{12}\right)+r_{1} f_{1}(n)+\cdots+r_{27} f_{27}(n) .
\end{aligned}
$$

In particular,

$$
\begin{aligned}
c(2 n)= & -c_{1} \sigma_{15}(2 n)-c_{2} \sigma_{15}(n)-c_{4} \sigma_{15}\left(\frac{n}{2}\right)-\left(16385 c_{3}+c_{6}\right) \sigma_{15}\left(\frac{n}{3}\right) \\
& -\left(c_{12}-16384 c_{3}\right) \sigma_{15}\left(\frac{n}{6}\right)+r_{1} f_{1}(2 n)+\cdots+r_{14} f_{14}(2 n),
\end{aligned}
$$




$$
c(2 n-1)=-c_{1} \sigma_{15}(2 n-1)-c_{3} \sigma_{15}\left(\frac{2 n-1}{3}\right)+r_{15} f_{15}(2 n-1)+\cdots+r_{27} f_{27}(2 n-1),
$$

for $n \in \mathbb{N}$.

Proof. It follows from (6)-(11) that

$$
\begin{gathered}
a_{1}+2 a_{2}+3 a_{3}+4 a_{4}+6 a_{6}+12 a_{12}=24 b_{1}, \\
a_{1}+a_{2}+a_{3}+a_{4}+a_{6}+a_{12}=32, \\
-\frac{a_{1}}{6}-\frac{a_{2}}{3}-\frac{a_{3}}{6}-\frac{2 a_{4}}{3}-\frac{a_{6}}{3}-\frac{2 a_{12}}{3}=-b_{1}-b_{5} .
\end{gathered}
$$

Now we will use p-k parametrization of Alaca, Alaca and Williams, see [15]:

$$
p(q):=\frac{\varphi^{2}(q)-\varphi^{2}\left(q^{3}\right)}{2 \varphi^{2}\left(q^{3}\right)}, k(q):=\frac{\varphi^{3}\left(q^{3}\right)}{\varphi(q)},
$$

where the theta function $\varphi(q)$ is defined by

$$
\varphi(q)=\sum_{-\infty}^{\infty} q^{n^{2}}
$$

Setting $x=p$ in (12), and multiplying both sides by $k^{16}$ we obtain

$$
\begin{aligned}
& \frac{k^{16}}{2^{b_{1}+b_{5}}} p^{b_{1}}(1-p)^{b_{2}}(1+p)^{b_{3}}(1+2 p)^{b_{4}}(2+p)^{b_{5}} \\
= & \left(k_{0}+k_{1} p+k_{2} p^{2}+k_{3} p^{3}+k_{4} p^{4}+k_{5} p^{5}+k_{6} p^{6}+k_{7} p^{7}+k_{8} p^{8}+k_{9} p^{9}+k_{10} p^{10}\right. \\
& +k_{11} p^{11}+k_{12} p^{12}+k_{13} p^{13}+k_{14} p^{14}+k_{15} p^{15}+k_{16} p^{16}+k_{17} p^{17}+k_{18} p^{18}+k_{19} p^{19} \\
& +k_{20} p^{20}+k_{21} p^{21}+k_{22} p^{22}+k_{23} p^{23}+k_{24} p^{24}+k_{25} p^{25}+k_{26} p^{26}+k_{27} p^{27}+k_{28} p^{28} \\
& \left.+k_{29} p^{29}+k_{30} p^{30}+k_{31} p^{31}+k_{32} p^{32}\right) k^{16} .
\end{aligned}
$$

Alaca, Alaca and Williams [16] have established the following representations in terms of $p$ and $k$ :

$$
\begin{gathered}
\eta(q)=2^{-1 / 6} p^{1 / 24}(1-p)^{1 / 2}(1+p)^{1 / 6}(1+2 p)^{1 / 8}(2+p)^{1 / 8} k^{1 / 2}, \\
\eta\left(q^{2}\right)=2^{-1 / 3} p^{1 / 22}(1-p)^{1 / 4}(1+p)^{1 / 12}(1+2 p)^{1 / 4}(2+p)^{1 / 4} k^{1 / 2}, \\
\eta\left(q^{3}\right)=2^{-1 / 6} p^{1 / 8}(1-p)^{1 / 6}(1+p)^{1 / 2}(1+2 p)^{1 / 24}(2+p)^{1 / 24} k^{1 / 2}, \\
\eta\left(q^{4}\right)=2^{-2 / 3} p^{1 / 6}(1-p)^{1 / 8}(1+p)^{1 / 24}(1+2 p)^{1 / 8}(2+p)^{1 / 2} k^{1 / 2}, \\
\eta\left(q^{6}\right)=2^{-1 / 3} p^{1 / 4}(1-p)^{1 / 12}(1+p)^{1 / 4}(1+2 p)^{1 / 12}(2+p)^{1 / 12} k^{1 / 2}, \\
\eta\left(q^{12}\right)=2^{-2 / 3} p^{1 / 2}(1-p)^{1 / 24}(1+p)^{1 / 8}(1+2 p)^{1 / 24}(2+p)^{1 / 6} k^{1 / 2}, \\
E_{6}(q):=1-504 \sum_{n=1}^{\infty} \sigma_{5}(n) q^{n} \\
=\left(1-246 p-5532 p^{2}-38614 p^{3}-135369 p^{4}-276084 p^{5}-348024 p^{6}\right. \\
\left.-276084 p^{7}-135369 p^{8}-38614 p^{9}-5532 p^{10}-246 p^{11}+p^{12}\right) k^{6}, \\
E_{4}(q):=1+240 \sum_{n=1}^{\infty} \sigma_{3}(n) q^{n} \\
=\left(1+124 p+964 p^{2}+2788 p^{3}+3910 p^{4}+2788 p^{5}+964 p^{6}+124 p^{7}+p^{8}\right) k^{4} .
\end{gathered}
$$


Therefore, since

$$
E_{16}(q)=\frac{2000}{3617} E_{6}^{2}(q) E_{4}(q)+\frac{1617}{3617} E_{4}^{4}(q)
$$

we immediately obtain:

$$
\begin{aligned}
& E_{16}(q)=\left(p^{32}+\frac{66032}{3617} p^{31}+\frac{134229104}{3617} p^{30}+\frac{31279681360}{3617} p^{29}+\frac{1532766571768}{3617} p^{28}\right. \\
& +\frac{33608470215088}{3617} p^{27}+\frac{431880430212432}{3617} p^{26}+\frac{3705935707430928}{3617} p^{25} \\
& +\frac{22884510224189340}{3617} p^{24}+\frac{106630640734839600}{3617} p^{23}+\frac{387088285203107184}{3617} p^{22} \\
& +\frac{1119620321590028304}{3617} p^{21}+\frac{2622064678835063496}{3617} p^{20}+\frac{5029685780950817520}{3617} p^{19} \\
& +\frac{7966990925710242000}{3617} p^{18}+\frac{10477360107681574608}{3617} p^{17}+\frac{11475149226459112710}{3617} p^{16} \\
& +\frac{10477360107681574608}{3617} p^{15}+\frac{7966990925710242000}{3617} p^{14}+\frac{5029685780950817520}{3617} p^{13} \\
& +\frac{2622064678835063496}{3617} p^{12}+\frac{1119620321590028304}{3617} p^{11}+\frac{387088285203107184}{3617} p^{10} \\
& +\frac{106630640734839600}{3617} p^{9}+\frac{22884510224189340}{3617} p^{8}+\frac{3705935707430928}{3617} p^{7} \\
& +\frac{431880430212432}{3617} p^{6}+\frac{33608470215088}{3617} p^{5}+\frac{1532766571768}{3617} p^{4} \\
& \left.+\frac{31279681360}{3617} p^{3}+\frac{134229104}{3617} p^{2}+\frac{66032}{3617} p+1\right) k^{16} \text {, } \\
& E_{16}\left(q^{2}\right)=\left(p^{32}+16 p^{31}+\frac{409184}{3617} p^{30}+\frac{1652680}{3617} p^{29}+\frac{37454668}{3617} p^{28}\right. \\
& +\frac{473232088}{3617} p^{27}+\frac{6483541032}{3617} p^{26}+\frac{56895857928}{3617} p^{25} \\
& +\frac{349784703630}{3617} p^{24}+\frac{1625990384280}{3617} p^{23}+\frac{5904566270904}{3617} p^{22} \\
& +\frac{17085449814024}{3617} p^{21} \frac{40012993999716}{3617} p^{20}+\frac{76746190229880}{3617} p^{19} \\
& +\frac{121562792943720}{3617} p^{18}+\frac{159871907133288}{3617} p^{17}+\frac{175100498074755}{3617} p^{16} \\
& +\frac{159871907133288}{3617} p^{15}+\frac{121562792943720}{3617} p^{14}+\frac{76746190229880}{3617} p^{13} \\
& +\frac{40012993999716}{3617} p^{12} \frac{17085449814024}{3617} p^{11}+\frac{5904566270904}{3617} p^{10} \\
& +\frac{1625990384280}{3617} p^{9}+\frac{349784703630}{3617} p^{8}+\frac{56895857928}{3617} p^{7} \\
& +\frac{6483541032}{3617} p^{6}+\frac{473232088}{3617} p^{5}+\frac{37454668}{3617} p^{4}+\frac{1652680}{3617} p^{3} \\
& \left.+\frac{409184}{3617} p^{2}+16 p+1\right) k^{16}
\end{aligned}
$$




$$
\begin{aligned}
& E_{16}\left(q^{3}\right)=\left(p^{32}+16 p^{31}+112 p^{30}+\frac{1593520}{3617} p^{29}+\frac{3661048}{3617} p^{28}+\frac{4034128}{3617} p^{27}\right. \\
& +\frac{6253392}{3617} p^{26}+\frac{94495728}{3617} p^{25}+\frac{584550300}{3617} p^{24}+\frac{2266437840}{3617} p^{23} \\
& +\frac{8171962224}{3617} p^{22}+\frac{26676060144}{3617} p^{21}+\frac{64864877256}{3617} p^{20} \\
& +\frac{116064498960}{3617} p^{19}+\frac{175509366480}{3617} p^{18}+\frac{243644509488}{3617} p^{17} \\
& +\frac{279458260230}{3617} p^{16}+\frac{243644509488}{3617} p^{15}+\frac{175509366480}{3617} p^{14} \\
& +\frac{116064498960}{3617} p^{13}+\frac{64864877256}{3617} p^{12}+\frac{26676060144}{3617} p^{11} \\
& +\frac{8171962224}{3617} p^{10}+\frac{2266437840}{3617} p^{9}+\frac{584550300}{3617} p^{8}+\frac{94495728}{3617} p^{7} \\
& \left.+\frac{6253392}{3617} p^{6}+\frac{4034128}{3617} p^{5}+\frac{3661048}{3617} p^{4}+\frac{1593520}{3617} p^{3}+112 p^{2}+16 p+1\right) k^{16} \\
& E_{16}\left(q^{4}\right)=\left(\frac{1}{65536} p^{32}+\frac{3107}{14815232} p^{31}+\frac{8373509}{14815232} p^{30}-\frac{426024575}{3703808} p^{29}+\frac{21371210189}{7407616} p^{28}\right. \\
& -\frac{2780210933}{231488} p^{27}-\frac{26515040733}{925952} p^{26}+\frac{46668005109}{462976} p^{25}+\frac{318947423355}{1851904} p^{24} \\
& -\frac{132759215175}{462976} p^{23}-\frac{243776400843}{462976} p^{22}+\frac{49438651083}{115744} p^{21}+\frac{265269995439}{231488} p^{20} \\
& +\frac{31565989785}{115744} p^{19}-\frac{6536535195}{14468} p^{18}+\frac{20783548953}{57872} p^{17}+\frac{573453480645}{462976} p^{16} \\
& +\frac{58547998863}{57872} p^{15}+\frac{24046100565}{57872} p^{14}+\frac{2526073485}{14468} p^{13}+\frac{4307305053}{28936} p^{12} \\
& +\frac{751890993}{7234} p^{11}+\frac{152474619}{3617} p^{10}+\frac{22732365}{3617} p^{9}-\frac{69921135}{14468} p^{8}-\frac{15090777}{3617} p^{7} \\
& \left.-\frac{2659113}{3617} p^{6}+\frac{3862768}{3617} p^{5}+\frac{3632488}{3617} p^{4}+440 p^{3}+112 p^{2}+16 p+1\right) k^{16} \\
& E_{16}\left(q^{6}\right)=\left(p^{32}+16 p^{31}+112 p^{30}+440 p^{29}+1004 p^{28}+1064 p^{27}-\frac{2745048}{3617} p^{26}\right. \\
& -\frac{15372552}{3617} p^{25}-\frac{20069490}{3617} p^{24}-\frac{2603160}{3617} p^{23}+\frac{25848504}{3617} p^{22} \\
& +\frac{33201144}{3617} p^{21}+\frac{8374116}{3617} p^{20}-\frac{19626360}{3617} p^{19}-\frac{18930840}{3617} p^{18} \\
& +\frac{4834968}{3617} p^{17}+\frac{18832515}{3617} p^{16}+\frac{4834968}{3617} p^{15}-\frac{18930840}{3617} p^{14} \\
& -\frac{19626360}{3617} p^{13}+\frac{8374116}{3617} p^{12}+\frac{33201144}{3617} p^{11}+\frac{25848504}{3617} p^{10} \\
& -\frac{2603160}{3617} p^{9}-\frac{20069490}{3617} p^{8}-\frac{15372552}{3617} p^{7}-\frac{2745048}{3617} p^{6} \\
& \left.+1064 p^{5}+1004 p^{4}+440 p^{3}+112 p^{2}+16 p+1\right) k^{16}
\end{aligned}
$$




$$
\begin{aligned}
E_{16}\left(q^{12}\right)= & \left(\frac{1}{65536} p^{32}+\frac{1}{4096} p^{31}+\frac{7}{4096} p^{30}+\frac{24835}{3703808} p^{29}\right. \\
& +\frac{112559}{7407616} p^{28}+\frac{3577}{231488} p^{27}+\frac{19407}{925952} p^{26}+\frac{179709}{462976} p^{25} \\
& +\frac{4552575}{1851904} p^{24}+\frac{2646075}{462976} p^{23}-\frac{4566453}{462976} p^{22}-\frac{11870997}{115744} p^{21} \\
& -\frac{64064691}{231488} p^{20}-\frac{24289965}{115744} p^{19}+\frac{40724415}{57872} p^{18} \\
& +\frac{127224993}{57872} p^{17}+\frac{932409285}{462976} p^{16}-\frac{109346667}{57872} p^{15} \\
& -\frac{386476065}{57872} p^{14}-\frac{82555605}{14468} p^{13}+\frac{67084983}{28936} p^{12} \\
& +\frac{18339}{2} p^{11}+7122 p^{10}-735 p^{9}-\frac{22215}{4} p^{8}-4251 p^{7} \\
& \left.-759 p^{6}+1064 p^{5}+1004 p^{4}+440 p^{3}+112 p^{2}+16 p+1\right) k^{16} .
\end{aligned}
$$

It is easy to check the following expressions by (20)-(25)

$$
\begin{aligned}
f_{1}:= & \sum_{n=0}^{\infty} f_{1}(n)=\frac{\eta^{18}(4 z) \eta^{16}(6 z) \eta^{10}(12 z)}{\eta^{12}(2 z)} \\
= & \left(-\frac{1}{524288} p^{27}-\frac{45}{1048576} p^{26}-\frac{29}{65536} p^{25}\right. \\
& -\frac{2897}{1048576} p^{24}-\frac{761}{65536} p^{23}-\frac{36143}{1048576} p^{22} \\
& -\frac{4809}{65536} p^{21}-\frac{115427}{1048576} p^{20}-\frac{55863}{524288} p^{19} \\
& -\frac{2653}{65536} p^{18}+\frac{1845}{32768} p^{17}+\frac{3859}{32768} p^{16}+\frac{1823}{16384} p^{15} \\
& \left.+\frac{133}{2048} p^{14}+\frac{49}{2048} p^{13}+\frac{21}{4096} p^{12}+\frac{1}{2048} p^{11}\right) k^{16}, \\
f_{2}= & \sum_{n=}^{\infty} f_{2}(n)=\frac{\eta^{20}(4 z) \eta^{2}(6 z) \eta^{20}(12 z)}{\eta^{10}(2 z)} \\
= & -\frac{1}{8388608} p^{29}-\frac{49}{16777216} p^{28}-\frac{69}{2097152} p^{27} \\
& -\frac{945}{4194304} p^{26} \frac{4377}{4194304} p^{25}-\frac{57639}{16777216} p^{2422} \\
& -\frac{68717}{8388608} p^{23}-\frac{58817}{4194304} p-\frac{34155}{2097152} p^{21} \\
& -\frac{5291}{524288} p^{20}+\frac{847}{262144} p^{19}+\frac{1959}{131072} p^{18}+\frac{1137}{65536} p^{17} \\
& \left.+\frac{765}{65536} p^{16}+\frac{159}{32768} p^{15}+\frac{19}{16384} p^{14}+\frac{1}{8192} p^{13}\right) k^{16},
\end{aligned}
$$




$$
\begin{aligned}
& f_{3}=\sum_{n=0}^{\infty} f_{3}(n)=\frac{\eta^{15}(4 z) \eta^{7}(6 z) \eta^{19}(12 z)}{\eta^{9}(2 z)} \\
& =\left(-\frac{1}{2097152} p^{28}-\frac{43}{4194304} p^{27}-\frac{421}{4194304} p^{26}-\frac{619}{1048576} p^{25}-\frac{2425}{1048576} p^{24}\right. \\
& -\frac{26443}{4194304} p^{23}-\frac{50501}{4194304} p^{22}-\frac{32463}{2097152} p^{21}-\frac{2925}{262144} p^{20}+\frac{17}{16384} p^{19} \\
& \left.+\frac{1709}{131072} p^{18}+\frac{1075}{65536} p^{17}+\frac{187}{16384} p^{16}+\frac{79}{16384} p^{15}+\frac{19}{16384} p^{14}+\frac{1}{8192} p^{13}\right) k^{16} \text {, } \\
& f_{4}=\sum_{n=0}^{\infty} f_{4}(n)=\frac{\eta^{18}(12 z) \eta^{10}(4 z) \eta^{12}(6 z)}{\eta^{8}(2 z)}=\left(-\frac{1}{524288} p^{27}-\frac{37}{1048576} p^{26}\right. \\
& -\frac{77}{262144} p^{25}-\frac{1517}{1048576} p^{24}-\frac{1219}{262144} p^{23}-\frac{10571}{1048576} p^{22}-\frac{3789}{262144} p^{21} \\
& -\frac{12519}{1048576} p^{20}-\frac{513}{524288} p^{19}+\frac{2933}{262144} p^{18}+\frac{2027}{131072} p^{17} \\
& \left.+\frac{731}{65536} p^{16}+\frac{157}{32768} p^{15}+\frac{19}{16384} p^{14}+\frac{1}{8192} p^{13}\right) k^{16}, \\
& f_{5}=\sum_{n=0}^{\infty} f_{5}(n)=\frac{\eta^{5}(4 z) \eta^{17}(6 z) \eta^{17}(12 z)}{\eta^{7}(2 z)}=\left(-\frac{1}{131072} p^{26}-\frac{31}{262144} p^{25}-\frac{213}{262144} p^{24}\right. \\
& -\frac{849}{262144} p^{23}-\frac{2145}{262144} p^{22}-\frac{3471}{262144} p^{21}-\frac{3263}{262144} p^{20}-\frac{739}{262144} p^{19}+\frac{2463}{262144} p^{18} \\
& \left.+\frac{1905}{131072} p^{17}+\frac{357}{32768} p^{16}+\frac{39}{8192} p^{15}+\frac{19}{16384} p^{14}+\frac{1}{8192} p^{13}\right) k^{16}, \\
& f_{6}=\sum_{n=0}^{\infty} f_{6}(n)=\frac{\eta^{17}(4 z) \eta^{17}(6 z) \eta^{5}(12 z)}{\eta^{7}(2 z)} \\
& =\left(\frac{1}{65536} p^{27}+\frac{11}{32768} p^{26}+\frac{881}{262144} p^{25}+\frac{5285}{262144} p^{24}+\frac{20991}{262144} p^{23}\right. \\
& +\frac{57213}{262144} p^{22}+\frac{105569}{262144} p^{21}+\frac{117767}{262144} p^{20}+\frac{31081}{262144} p^{19}-\frac{142257}{262144} p^{18} \\
& -\frac{136127}{131072} p^{17}-\frac{14399}{16384} p^{16}-\frac{397}{2048} p^{15}+\frac{3249}{8192} p^{14} \\
& \left.+\frac{2103}{4096} p^{13}+\frac{161}{512} p^{12}+\frac{115}{1024} p^{11}+\frac{23}{1024} p^{10}+\frac{1}{512} p^{9}\right) k^{16}, \\
& f_{7}=\sum_{n=0}^{\infty} f_{7}(n)=\frac{\eta^{19}(4 z) \eta^{15}(6 z) \eta^{15}(12 z)}{\eta^{17}(2 z)} \\
& =\left(\frac{1}{4194304} p^{27}+\frac{23}{4194304} p^{26}+\frac{61}{1048576} p^{25}+\frac{791}{2097152} p^{24}+\frac{7001}{4194304} p^{23}\right. \\
& +\frac{22363}{4194304} p^{22}+\frac{26577}{2097152} p^{21}+\frac{5967}{262144} p^{20}+\frac{1017}{32768} p^{19}+\frac{4189}{131072} p^{18} \\
& \left.+\frac{1603}{65536} p^{17}+\frac{221}{16384} p^{16}+\frac{83}{16384} p^{15}+\frac{19}{16384} p^{14}+\frac{1}{8192} p^{13}\right) k^{16},
\end{aligned}
$$




$$
\begin{aligned}
& f_{8}=\sum_{n=0}^{\infty} f_{8}(n)=\frac{\eta^{17}(2 z) \eta^{17}(4 z) \eta^{5}(12 z)}{\eta^{7}(6 z)} \\
& =\left(\frac{1}{4096} p^{31}+\frac{3}{512} p^{30}+\frac{1031}{16384} p^{29}+\frac{6445}{16384} p^{28}+\frac{101019}{65536} p^{27}\right. \\
& +\frac{120001}{32768} p^{26}+\frac{984145}{262144} p^{25}-\frac{1537659}{262144} p^{24}-\frac{7102105}{262144} p^{23} \\
& -\frac{9302339}{262144} p^{22}+\frac{2219505}{262144} p^{21}+\frac{23214863}{262144} p^{20}+\frac{26270329}{262144} p^{19} \\
& -\frac{5377425}{262144} p^{18}-\frac{20003423}{131072} p^{17}-\frac{3918925}{32768} p^{16}+\frac{346941}{8192} p^{15} \\
& +\frac{2088461}{16384} p^{14}+\frac{512615}{8192} p^{13}-\frac{7401}{256} p^{12}-\frac{47239}{1024} p^{11} \\
& \left.-\frac{16583}{1024} p^{10}+\frac{2319}{512} p^{9}+\frac{775}{128} p^{8}+\frac{37}{16} p^{7}+\frac{27}{64} p^{6}+\frac{1}{32} p^{5}\right) k^{16}, \\
& f_{9}=\sum_{n=0}^{\infty} f_{9}(n)=\frac{\eta^{20}(2 z) \eta^{14}(4 z) \eta^{14}(12 z)}{\eta^{16}(6 z)} \\
& =\left(\frac{1}{16384} p^{32}+\frac{23}{16384} p^{31}+\frac{939}{65536} p^{30}+\frac{2753}{32768} p^{29}+\frac{78995}{262144} p^{28}\right. \\
& +\frac{161007}{262144} p^{27}+\frac{340117}{1048576} p^{26}-\frac{117361}{65536} p^{25}-\frac{5224329}{1048576} p^{24} \\
& -\frac{2039005}{524288} p^{23}+\frac{6297515}{1048576} p^{22}+\frac{4229337}{262144} p^{21}+\frac{9352981}{1048576} p^{20} \\
& -\frac{7365203}{524288} p^{19}-\frac{3159555}{131072} p^{18}-\frac{379685}{65536} p^{17}+\frac{1073567}{65536} p^{16} \\
& +\frac{507447}{32768} p^{15}+\frac{323}{2048} p^{14}-\frac{15125}{2048} p^{13}-\frac{16989}{4096} p^{12} \\
& \left.+\frac{203}{2048} p^{11}+\frac{529}{512} p^{10}+\frac{123}{256} p^{9}+\frac{25}{256} p^{8}+\frac{1}{128} p^{7}\right) k^{16}, \\
& f_{10}=\sum_{n=0}^{\infty} f_{10}(n)=\frac{\eta^{16}(4 z) \eta^{18}(6 z)}{\eta^{2}(2 z)} \\
& =\left(-\frac{1}{8192} p^{27}-\frac{43}{16384} p^{26}-\frac{835}{32768} p^{25}-\frac{9601}{65536} p^{24}\right. \\
& -\frac{4477}{8192} p^{23}-\frac{44075}{32768} p^{22}-\frac{66467}{32768} p^{21}-\frac{4547}{4096} p^{20} \\
& +\frac{80587}{32768} p^{19}+\frac{216681}{32768} p^{18}+\frac{108333}{16384} p^{17}+\frac{46257}{65536} p^{16} \\
& -\frac{200503}{32768} p^{15}-\frac{30571}{4096} p^{14}-\frac{6751}{2048} p^{13}+\frac{2303}{2048} p^{12} \\
& \left.+\frac{2471}{1024} p^{11}+\frac{391}{256} p^{10}+\frac{67}{128} p^{9}+\frac{25}{256} p^{8}+\frac{1}{128} p^{7}\right) k^{16},
\end{aligned}
$$




$$
\begin{aligned}
& f_{11}=\sum_{n=0}^{\infty} f_{11}(n)=\frac{\eta^{20}(2 z) \eta^{14}(4 z) \eta^{8}(6 z)}{\eta^{10}(12 z)} \\
& =\left(-\frac{1}{32} p^{29}-\frac{43}{64} p^{28}-\frac{813}{128} p^{27}-\frac{8683}{256} p^{26}-\frac{54571}{512} p^{25}\right. \\
& -\frac{169029}{1024} p^{24}+\frac{166177}{2048} p^{23}+\frac{3773287}{4096} p^{22}+\frac{1665717}{1024} p^{21} \\
& +\frac{541157}{4096} p^{20}-\frac{8039515}{2048} p^{19}-\frac{11541531}{2048} p^{18}+\frac{52297}{128} p^{17} \\
& +\frac{19076567}{2048} p^{16}+\frac{17486919}{2048} p^{15}-\frac{13136125}{4096} p^{14} \\
& -\frac{11342417}{1024} p^{13}-\frac{22863279}{4096} p^{12}+\frac{8459683}{2048} p^{11} \\
& +\frac{6273043}{1024} p^{10}+\frac{828501}{512} p^{9}-\frac{220715}{128} p^{8}-\frac{98825}{64} p^{7} \\
& \left.-\frac{10071}{32} p^{6}+\frac{3281}{16} p^{5}+\frac{2581}{16} p^{4}+\frac{399}{8} p^{3}+\frac{31}{4} p^{2}+\frac{1}{2} p\right) k^{16}, \\
& f_{12}=\sum_{n=0}^{\infty} f_{12}(n)=\eta^{20}(2 z) \eta^{2}(4 z) \eta^{8}(6 z) \eta^{2}(12 z) \\
& =\left(\frac{1}{64} p^{28}+\frac{15}{64} p^{27}+\frac{371}{256} p^{26}+\frac{569}{128} p^{25}+\frac{5211}{1024} p^{24}-\frac{9241}{1024} p^{23}\right. \\
& -\frac{153891}{4096} p^{22}-\frac{8919}{256} p^{21}+\frac{179109}{4096} p^{20}+\frac{251003}{2048} p^{19}+\frac{113727}{2048} p^{18} \\
& -\frac{30079}{256} p^{17}-\frac{329953}{2048} p^{16}-\frac{2349}{256} p^{15}+\frac{497249}{4096} p^{14}+\frac{42867}{512} p^{13} \\
& -\frac{63711}{4096} p^{12}-\frac{93897}{2048} p^{11}-\frac{19619}{1024} p^{10}+\frac{1587}{512} p^{9}+\frac{1475}{256} p^{8} \\
& \left.+\frac{293}{128} p^{7}+\frac{27}{64} p^{6}+\frac{1}{32} p^{5}\right) k^{16}, \\
& f_{14}=\sum_{n=0}^{\infty} f_{14}(n)=\frac{\eta^{16}(2 z) \eta^{10}(4 z) \eta^{18}(12 z)}{\eta^{12}(6 z)} \\
& =\left(-\frac{1}{32768} p^{31}-\frac{41}{65536} p^{30}-\frac{369}{65536} p^{29}-\frac{3755}{131072} p^{28}\right. \\
& -\frac{45365}{524288} p^{27}-\frac{142317}{1048576} p^{26}+\frac{2541}{262144} p^{25}+\frac{523731}{1048576} p^{24} \\
& +\frac{238005}{262144} p^{23}+\frac{289525}{1048576} p^{22}-\frac{358967}{262144} p^{21}-\frac{2160639}{1048576} p^{20} \\
& -\frac{178493}{524288} p^{19}+\frac{503675}{262144} p^{18}+\frac{246645}{131072} p^{17}-\frac{51}{32768} p^{16} \\
& -\frac{18129}{16384} p^{15}-\frac{5643}{8192} p^{14}+\frac{25}{4096} p^{13}+\frac{805}{4096} p^{12}+\frac{207}{2048} p^{11} \\
& \left.+\frac{23}{1024} p^{10}+\frac{1}{512} p^{9}\right) k^{16} \text {, }
\end{aligned}
$$




$$
\begin{aligned}
& f_{15}=\sum_{n=0}^{\infty} f_{15}(n)=\frac{\eta^{19}(4 z) \eta^{9}(6 z) \eta^{15}(12 z)}{\eta^{11}(2 z)} \\
& =\left(-\frac{1}{2097152} p^{28}-\frac{47}{4194304} p^{27}-\frac{507}{4194304} p^{26}-\frac{1659}{2097152} p^{25}\right. \\
& -\frac{3663}{1048576} p^{24}-\frac{45843}{4194304} p^{23}-\frac{103387}{4194304} p^{22}-\frac{20741}{524288} p^{21} \\
& -\frac{44163}{1048576} p^{20}-\frac{2789}{131072} p^{19}+\frac{1981}{131072} p^{18}+\frac{87}{2048} p^{17} \\
& \left.+\frac{1449}{32768} p^{16}+\frac{453}{16384} p^{15}+\frac{177}{16384} p^{14}+\frac{5}{2048} p^{13}+\frac{1}{4096} p^{12}\right) k^{16}, \\
& f_{16}=\sum_{n=0}^{\infty} f_{16}(n)=\frac{\eta^{14}(4 z) \eta^{14}(6 z) \eta^{14}(12 z)}{\eta^{10}(2 z)} \\
& =\left(-\frac{1}{524288} p^{27}-\frac{41}{1048576} p^{26}-\frac{191}{524288} p^{25}-\frac{2133}{1048576} p^{24}\right. \\
& -\frac{3955}{524288} p^{23}-\frac{20323}{1048576} p^{22}-\frac{18149}{524288} p^{21}-\frac{42831}{1048576} p^{20} \\
& -\frac{1629}{65536} p^{19}+\frac{605}{65536} p^{18}+\frac{155}{4096} p^{17}+\frac{1379}{32768} p^{16} \\
& \left.+\frac{111}{4096} p^{15}+\frac{11}{1024} p^{14}+\frac{5}{2048} p^{13}+\frac{1}{4096} p^{12}\right) k^{16}, \\
& f_{17}=\sum_{n=0}^{\infty} f_{17}(n)=\frac{\eta^{15}(4 z) \eta^{13}(6 z) \eta^{19}(12 z)}{\eta^{15}(2 z)} \\
& =\left(\frac{1}{4194304} p^{27}+\frac{21}{4194304} p^{26}+\frac{101}{2097152} p^{25}+\frac{589}{2097152} p^{24}\right. \\
& +\frac{4645}{4194304} p^{23}+\frac{13073}{4194304} p^{22}+\frac{211}{32768} p^{21}+\frac{2591}{262144} p^{20} \\
& +\frac{1477}{131072} p^{19}+\frac{1235}{131072} p^{18}+\frac{23}{4096} p^{17} \\
& \left.+\frac{37}{16384} p^{16}+\frac{9}{16384} p^{15}+\frac{1}{16384} p^{14}\right) k^{16}, \\
& f_{18}=\sum_{n=0}^{\infty} f_{18}(n)=\frac{\eta^{9}(4 z) \eta^{19}(6 z) \eta^{13}(12 z)}{\eta^{9}(2 z)} \\
& =\left(-\frac{1}{131072} p^{26}-\frac{35}{262144} p^{25}-\frac{275}{262144} p^{24}-\frac{1275}{262144} p^{23}\right. \\
& -\frac{3843}{262144} p^{22}-\frac{7761}{262144} p^{21}-\frac{10205}{262144} p^{20}-\frac{7265}{262144} p^{19} \\
& +\frac{985}{262144} p^{18}+\frac{273}{8192} p^{17}+\frac{2619}{65536} p^{16}+\frac{435}{16384} p^{15} \\
& \left.+\frac{175}{16384} p^{14}+\frac{5}{2048} p^{13}+\frac{1}{4096} p^{12}\right) k^{16},
\end{aligned}
$$




$$
\begin{aligned}
& f_{19}=\sum_{n=0}^{\infty} f_{19}(n)=\frac{\eta^{18}(4 z) \eta^{10}(6 z) \eta^{10}(12 z)}{\eta^{6}(2 z)} \\
& =\left(\frac{1}{262144} p^{28}+\frac{23}{262144} p^{27}+\frac{965}{1048576} p^{26}+\frac{3041}{524288} p^{25}\right. \\
& +\frac{25479}{1048576} p^{24}+\frac{18429}{262144} p^{23}+\frac{146279}{1048576} p^{22}+\frac{91313}{524288} p^{21} \\
& +\frac{83989}{1048576} p^{20}-\frac{41021}{262144} p^{19}-\frac{98171}{262144} p^{18}-\frac{1493}{4096} p^{17} \\
& -\frac{4043}{32768} p^{16}+\frac{1029}{8192} p^{15}+\frac{1647}{8192} p^{14}+\frac{275}{2048} p^{13} \\
& \left.+\frac{209}{4096} p^{12}+\frac{11}{1024} p^{11}+\frac{1}{1024} p^{10}\right) k^{16}, \\
& f_{20}=\sum_{n=0}^{\infty} f_{20}(n)=\frac{\eta^{20}(2 z) \eta^{8}(4 z) \eta^{20}(6 z)}{\eta^{16}(12 z)} \\
& =\left(-\frac{1}{2} p^{27}-\frac{35}{4} p^{26}-\frac{521}{8} p^{25}-\frac{4111}{16} p^{24}-\frac{15975}{32} p^{23}+\frac{327}{64} p^{22}\right. \\
& +\frac{297757}{128} p^{21}+\frac{1195499}{256} p^{20}+\frac{106085}{128} p^{19}-\frac{685735}{64} p^{18} \\
& -\frac{62285}{4} p^{17}+\frac{435395}{256} p^{16}+\frac{1648595}{64} p^{15}+\frac{1378995}{64} p^{14} \\
& -\frac{1378995}{128} p^{13}-\frac{7434175}{256} p^{12}-\frac{1547495}{128} p^{11}+\frac{776305}{64} p^{10} \\
& +\frac{951275}{64} p^{9}+\frac{734305}{256} p^{8}-\frac{73567}{16} p^{7}-\frac{56545}{16} p^{6}-\frac{2247}{4} p^{5} \\
& \left.+\frac{4203}{8} p^{4}+365 p^{3}+107 p^{2}+16 p+1\right) k^{16}, \\
& f_{21}=\sum_{n=0}^{\infty} f_{21}(n)=\frac{\eta^{20}(2 z) \eta^{20}(4 z)}{\eta^{4}(6 z) \eta^{4}(12 z)} \\
& =\left(-\frac{1}{512} p^{31}-\frac{51}{1024} p^{30}-\frac{1169}{2048} p^{29}-\frac{15687}{4096} p^{28}-\frac{133119}{8192} p^{27}\right. \\
& -\frac{697473}{16384} p^{26}-\frac{1714923}{32768} p^{25}+\frac{3811059}{65536} p^{24}+\frac{11844819}{32768} p^{23} \\
& +\frac{18633153}{32768} p^{22}-\frac{456813}{32768} p^{21}-\frac{23799867}{16384} p^{20}-\frac{67065705}{32768} p^{19} \\
& +\frac{3013731}{32768} p^{18}+\frac{27673335}{8192} p^{17}+\frac{219131721}{65536} p^{16}-\frac{14731353}{16384} p^{15} \\
& -\frac{68551245}{16384} p^{14}-\frac{5076561}{2048} p^{13}+\frac{5524785}{4096} p^{12}+\frac{2546313}{1024} p^{11} \\
& +\frac{856899}{1024} p^{10}-\frac{40113}{64} p^{9}-\frac{170541}{256} p^{8}-\frac{10647}{64} p^{7} \\
& \left.+\frac{4977}{64} p^{6}+\frac{567}{8} p^{5}+\frac{371}{16} p^{4}+\frac{15}{4} p^{3}+\frac{1}{4} p^{2}\right) k^{16},
\end{aligned}
$$




$$
\begin{aligned}
& f_{22}=\sum_{n=0}^{\infty} f_{22}(n)=\frac{\eta^{20}(4 z) \eta^{20}(12 z)}{\eta^{4}(2 z) \eta^{4}(6 z)} \\
& =\left(\frac{1}{4194304} p^{30}+\frac{25}{4194304} p^{29}+\frac{1145}{16777216} p^{28}\right. \\
& +\frac{495}{1048576} p^{27}+\frac{18341}{8388608} p^{26}+\frac{29669}{4194304} p^{25} \\
& +\frac{269025}{16777216} p^{24}+\frac{50735}{2097152} p^{23}+\frac{39853}{2097152} p^{22} \\
& -\frac{4411}{524288} p^{21}-\frac{46849}{1048576} p^{20}-\frac{3785}{65536} p^{19} \\
& -\frac{2115}{65536} p^{18}+\frac{67}{8192} p^{17}+\frac{1903}{65536} p^{16}+\frac{195}{8192} p^{15} \\
& \left.+\frac{85}{8192} p^{14}+\frac{5}{2048} p^{13}+\frac{1}{4096} p^{12}\right) k^{16}, \\
& f_{23}=\sum_{n=0}^{\infty} f_{23}(n)=\frac{\eta^{20}(4 z) \eta^{20}(6 z)}{\eta^{4}(2 z) \eta^{4}(12 z)} \\
& =\left(-\frac{1}{8192} p^{27}-\frac{47}{16384} p^{26}-\frac{1007}{32768} p^{25}-\frac{12941}{65536} p^{24}\right. \\
& -\frac{27509}{32768} p^{23}-\frac{79891}{32768} p^{22}-\frac{154617}{32768} p^{21}-\frac{84655}{16384} p^{20} \\
& +\frac{7835}{32768} p^{19}+\frac{377855}{32768} p^{18}+\frac{162507}{8192} p^{17}+\frac{912921}{65536} p^{16} \\
& -\frac{77123}{16384} p^{15}-\frac{322787}{16384} p^{14}-\frac{18661}{1024} p^{13}-\frac{11199}{2048} p^{12} \\
& +\frac{2387}{512} p^{11}+\frac{3253}{512} p^{10}+\frac{229}{64} p^{9}+\frac{293}{256} p^{8} \\
& \left.+\frac{13}{64} p^{7}+\frac{1}{64} p^{6}\right) k^{16}, \\
& f_{24}=\sum_{n=0}^{\infty} f_{24}(n)=\frac{\eta^{15}(4 z) \eta(6 z) \eta^{19}(12 z)}{\eta^{3}(2 z)} \\
& =\left(\frac{1}{1048576} p^{29}+\frac{11}{524288} p^{28}+\frac{877}{4194304} p^{27}+\frac{5205}{4194304} p^{26}\right. \\
& +\frac{10137}{2097152} p^{25}+\frac{26721}{2097152} p^{24}+\frac{92837}{4194304} p^{23}+\frac{89789}{4194304} p^{22} \\
& -\frac{725}{524288} p^{21}-\frac{39571}{1048576} p^{20}-\frac{7325}{131072} p^{19}-\frac{4619}{131072} p^{18} \\
& +\frac{9}{2048} p^{17}+\frac{885}{32768} p^{16}+\frac{381}{16384} p^{15}+\frac{169}{16384} p^{14} \\
& \left.+\frac{5}{2048} p^{13}+\frac{1}{4096} p^{12}\right) k^{16},
\end{aligned}
$$




$$
\begin{aligned}
& f_{25}=\sum_{n=0}^{\infty} f_{25}(n)=\frac{\eta^{20}(2 z) \eta^{20}(4 z)}{\eta^{4}(6 z) \eta^{4}(12 z)} \\
& =\left(-\frac{1}{8} p^{25}-\frac{9}{16} p^{24}-\frac{7}{16} p^{23}+\frac{55}{32} p^{22}+\frac{435}{128} p^{21}\right. \\
& -\frac{101}{256} p^{20}-\frac{781}{128} p^{19}-\frac{33}{8} p^{18}+\frac{115}{32} p^{17}+\frac{1475}{256} p^{16} \\
& +\frac{63}{64} p^{15}-\frac{41}{16} p^{14}-\frac{229}{128} p^{13}-\frac{15}{256} p^{12} \\
& \left.+\frac{55}{128} p^{11}+\frac{7}{32} p^{10}+\frac{3}{64} p^{9}+\frac{1}{256} p^{8}\right) k^{16}, \\
& f_{26}=\sum_{n=0}^{\infty} f_{26}(n)=\frac{\eta^{20}(2 z) \eta^{20}(6 z) \eta^{8}(12 z)}{\eta^{4}(4 z)} \\
& =\left(-\frac{1}{8} p^{28}-\frac{41}{16} p^{27}-\frac{735}{32} p^{26}-\frac{7369}{64} p^{25}-\frac{42461}{128} p^{24}\right. \\
& -\frac{108327}{256} p^{23}+\frac{261427}{512} p^{22}+\frac{3059933}{1024} p^{21} \\
& +\frac{4316289}{1024} p^{20}-\frac{1428553}{1024} p^{19}-\frac{12680767}{1024} p^{18} \\
& -\frac{6900279}{512} p^{17}+\frac{3095779}{512} p^{16}+\frac{13721761}{512} p^{15} \\
& +\frac{2279991}{128} p^{14}-\frac{14642143}{1024} p^{13}-\frac{29221507}{1024} p^{12} \\
& -\frac{9789933}{1024} p^{11}+\frac{13635953}{1024} p^{10}+\frac{921227}{64} p^{9} \\
& +\frac{560229}{256} p^{8}-\frac{153647}{32} p^{7}-\frac{111071}{32} p^{6} \\
& \left.-\frac{987}{2} p^{5}+\frac{4387}{8} p^{4}+\frac{1475}{4} p^{3}+\frac{429}{4} p^{2}+16 p+1\right) k^{16}, \\
& f_{27}=\sum_{n=0}^{\infty} f_{27}(n)=\frac{\eta^{19}(2 z) \eta^{13}(4 z) \eta^{15}(6 z)}{\eta^{15}(12 z)} \\
& =\left(-\frac{1}{32} p^{26}-\frac{15}{64} p^{25}-\frac{9}{16} p^{24}-\frac{1}{128} p^{23}\right. \\
& +\frac{1095}{512} p^{22}+\frac{2949}{1024} p^{21}-\frac{1435}{1024} p^{20} \\
& -\frac{6273}{1024} p^{19}-\frac{3279}{1024} p^{18}+\frac{2105}{512} p^{17}+\frac{2811}{512} p^{16} \\
& +\frac{315}{512} p^{15}-\frac{169}{64} p^{14}-\frac{1767}{1024} p^{13}-\frac{15}{1024} p^{12} \\
& \left.+\frac{451}{1024} p^{11}+\frac{225}{1024} p^{10}+\frac{3}{64} p^{9}+\frac{1}{256} p^{8}\right) k^{16} .
\end{aligned}
$$

Obviously, $f_{1}, \cdots, f_{27}$ are functions of $q$, see (3), (19). We see that

$\left\{f_{1}, \cdots, f_{27}\right\} \backslash\left\{f_{7}, f_{9}, f_{17}, f_{20}, f_{25}, f_{26}, f_{27}\right\} \in S_{16}\left(\Gamma_{0}(12)\right), \quad f_{7}, f_{9}, f_{17}, f_{20}, f_{25}, f_{26}, f_{27} \in M_{16}\left(\Gamma_{0}(12)\right) \backslash S_{16}\left(\Gamma_{0}(12)\right)$ by [17]. Now 


$$
\begin{aligned}
& \eta^{a_{1}}(z) \eta^{a_{2}}(2 z) \eta^{a_{3}}(3 z) \eta^{a_{4}}(4 z) \eta^{a_{6}}(6 z) \eta^{a_{12}}(12 z) \\
& =q^{b_{1}} \prod_{n=1}^{\infty}\left(1-q^{n}\right)^{a_{1}}\left(1-q^{2 n}\right)^{a_{2}}\left(1-q^{3 n}\right)^{a_{3}}\left(1-q^{4 n}\right)^{a_{4}}\left(1-q^{6 n}\right)^{a_{6}}\left(1-q^{12 n}\right)^{a_{12}} \\
& =2^{-\frac{a_{1}}{6}-\frac{a_{2}}{3}-\frac{a_{3}}{6}-\frac{2 a_{4}}{3}-\frac{a_{6}}{3}-\frac{2 a_{12}}{3}} p^{\frac{a_{1}}{24}+\frac{a_{2}}{12}+\frac{a_{3}}{8}+\frac{a_{4}}{6}+\frac{a_{6}}{4}+\frac{a_{12}}{2}} \\
& \cdot(1-p)^{\frac{a_{1}}{2}+\frac{a_{2}}{4}+\frac{a_{3}}{6}+\frac{a_{4}}{8}+\frac{a_{6}}{12}+\frac{a_{12}}{24}}(1+p)^{\frac{a_{1}}{6}+\frac{a_{2}}{12}+\frac{a_{3}}{2}+\frac{a_{4}}{24}+\frac{a_{6}}{4}+\frac{a_{12}}{8}}
\end{aligned}
$$

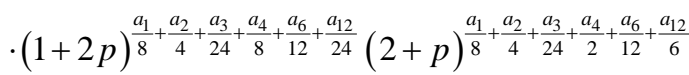

$$
\begin{aligned}
& \cdot k \frac{a_{1}+a_{2}+a_{3}+a_{4}+a_{6}+a_{12}}{2} \\
& =\frac{k^{16}}{2^{b_{1}+b_{5}}} p^{b_{1}}(1-p)^{b_{2}}(1+p)^{b_{3}}(1+2 p)^{b_{4}}(2+p)^{b_{5}} \\
& =k^{16}\left(k_{0}+k_{1} p+k_{2} p^{2}+k_{3} p^{3}+k_{4} p^{4}+k_{5} p^{5}\right. \\
& +k_{6} p^{6}+k_{7} p^{7}+k_{8} p^{8}+k_{9} p^{9}+k_{10} p^{10}+k_{11} p^{11} \\
& +k_{12} p^{12}+k_{13} p^{13}+k_{14} p^{14}+k_{15} p^{15}+k_{16} p^{16} \\
& +k_{17} p^{17}+k_{18} p^{18}+k_{19} p^{19}+k_{20} p^{20}+k_{21} p^{21} \\
& +k_{22} p^{22}+k_{23} p^{23}+k_{24} p^{24}+k_{25} p^{25}+k_{26} p^{26} \\
& \left.+k_{27} p^{27}+k_{28} p^{28}+k_{29} p^{29}+k_{30} p^{30}+k_{31} p^{31}+k_{32} p^{32}\right) \\
& =\frac{3617 c_{1}}{16320}\left(1+\frac{16320}{3617} \sum_{n=1}^{\infty} \sigma_{15}(n) q^{n}\right)+\frac{3617 c_{2}}{16320}\left(1+\frac{16320}{3617} \sum_{n=1}^{\infty} \sigma_{15}(n) q^{2 n}\right) \\
& +\frac{3617 c_{3}}{16320}\left(1+\frac{16320}{3617} \sum_{n=1}^{\infty} \sigma_{15}(n) q^{3 n}\right)+\frac{3617 c_{4}}{16320}\left(1+\frac{16320}{3617} \sum_{n=1}^{\infty} \sigma_{15}(n) q^{4 n}\right) \\
& +\frac{3617 c_{5}}{16320}\left(1+\frac{16320}{3617} \sum_{n=1}^{\infty} \sigma_{15}(n) q^{6 n}\right)+\frac{3617 c_{6}}{16320}\left(1+\frac{16320}{3617} \sum_{n=1}^{\infty} \sigma_{15}(n) q^{12 n}\right) \\
& +r_{1} q^{11} \prod_{n=1}^{\infty} \frac{\left(1-q^{4 n}\right)^{18}\left(1-q^{6 n}\right)^{16}\left(1-q^{12 n}\right)^{10}}{\left(1-q^{2 n}\right)^{12}}+r_{2} q^{13} \prod_{n=1}^{\infty} \frac{\left(1-q^{4 n}\right)^{20}\left(1-q^{6 n}\right)^{2}\left(1-q^{12}\right)^{20}}{\left(1-q^{2 n}\right)^{10}} \\
& +r_{3} q^{13} \prod_{n=1}^{\infty} \frac{\left(1-q^{4 n}\right)^{15}\left(1-q^{6 n}\right)^{7}\left(1-q^{12 n}\right)^{19}}{\left(1-q^{2 n}\right)^{9}}+r_{4} q^{13} \prod_{n=1}^{\infty} \frac{\left(1-q^{2 n}\right)^{10}\left(1-q^{4 n}\right)^{12}\left(1-q^{6 n}\right)^{18}}{\left(1-q^{12 n}\right)^{8}} \\
& +r_{5} q^{13} \prod_{n=1}^{\infty} \frac{\left(\left(1-q^{4 n}\right)^{7}\left(1-q^{6 n}\right)^{17}\left(1-q^{12 n}\right)^{17}\right.}{\left(1-q^{2 n}\right)^{7}}+r_{6} q^{9} \prod_{n=1}^{\infty} \frac{\left(1-q^{4 n}\right)^{17}\left(1-q^{6 n}\right)^{17}\left(1-q^{12 n}\right)^{5}}{\left(1-q^{2 n}\right)^{7}} \\
& +r_{7} q^{13} \prod_{n=1}^{\infty} \frac{\left(1-q^{4 n}\right)^{19}\left(1-q^{6 n}\right)^{15}\left(1-q^{12 n}\right)^{15}}{\left(1-q^{2 n}\right)^{17}}+r_{8} q^{5} \prod_{n=1}^{\infty} \frac{\left(1-q^{2 n}\right)^{17}\left(1-q^{4 n}\right)^{17}\left(1-q^{12 n}\right)^{5}}{\left(1-q^{6 n}\right)^{7}} \\
& +r_{9} q^{7} \prod_{n=1}^{\infty} \frac{\left(1-q^{2 n}\right)^{20}\left(1-q^{4 n}\right)^{14}\left(1-q^{12 n}\right)^{14}}{\left(1-q^{6 n}\right)^{16}}+r_{10} q^{7} \prod_{n=1}^{\infty} \frac{\left(1-q^{4 n}\right)^{16}\left(1-q^{6 n}\right)^{18}}{\left(1-q^{2 n}\right)^{2}} \\
& +r_{11} q \prod_{n=1}^{\infty} \frac{\left(1-q^{2 n}\right)^{20}\left(1-q^{4 n}\right)^{14}\left(1-q^{6 n}\right)^{8}}{\left(1-q^{12 n}\right)^{10}}+r_{12} q^{5} \prod_{n=1}^{\infty}\left(1-q^{2 n}\right)^{20}\left(1-q^{4 n}\right)^{2}\left(1-q^{6 n}\right)^{8}\left(1-q^{12 n}\right)^{2}
\end{aligned}
$$




$$
\begin{aligned}
& +r_{13} q^{13} \prod_{n=1}^{\infty} \frac{\left(1-q^{2 n}\right)^{2}\left(1-q^{6 n}\right)^{14}\left(1-q^{12 n}\right)^{20}}{\left(1-q^{4 n}\right)^{4}}+r_{14} q^{9} \prod_{n=1}^{\infty} \frac{\left(1-q^{2 n}\right)^{16}\left(1-q^{4 n}\right)^{10}\left(1-q^{12 n}\right)^{18}}{\left(1-q^{6 n}\right)^{12}} \\
& +r_{15} q^{12} \prod_{n=1}^{\infty} \frac{\left(1-q^{4 n}\right)^{19}\left(1-q^{6 n}\right)^{9}\left(1-q^{12 n}\right)^{15}}{\left(1-q^{2 n}\right)^{11}}+r_{16} q^{12} \prod_{n=1}^{\infty} \frac{\left(1-q^{4 n}\right)^{14}\left(1-q^{6 n}\right)^{14}\left(1-q^{12 n}\right)^{14}}{\left(1-q^{2 n}\right)^{10}} \\
& +r_{17} q^{14} \prod_{n=1}^{\infty} \frac{\left(1-q^{4 n}\right)^{15}\left(1-q^{6 n}\right)^{13}\left(1-q^{12 n}\right)^{19}}{\left(1-q^{2 n}\right)^{15}}+r_{18} q^{12} \prod_{n=1}^{\infty} \frac{\left(1-q^{4 n}\right)^{9}\left(1-q^{6 n}\right)^{19}\left(1-q^{12 n}\right)^{13}}{\left(1-q^{2 n}\right)^{9}} \\
& +r_{19} q^{10} \prod_{n=1}^{\infty} \frac{\left(1-q^{4 n}\right)^{18}\left(1-q^{6 n}\right)^{10}\left(1-q^{12 n}\right)^{10}}{\left(1-q^{2 n}\right)^{6}} \\
& +r_{20} \prod_{n=1}^{\infty} \frac{\left(1-q^{2 n}\right)^{20}\left(1-q^{4 n}\right)^{8}\left(1-q^{6 n}\right)^{20}}{\left(1-q^{12 n}\right)^{16}} \\
& +r_{21} q^{2} \prod_{n=1}^{\infty} \frac{\left(1-q^{2 n}\right)^{20}\left(1-q^{4 n}\right)^{20}}{\left(1-q^{6 n}\right)^{4}\left(1-q^{12 n}\right)^{4}} \\
& +r_{22} q^{12} \prod_{n=1}^{\infty} \frac{\left(1-q^{4 n}\right)^{20}\left(1-q^{12 n}\right)^{20}}{\left(1-q^{2 n}\right)^{4}\left(1-q^{6 n}\right)^{4}} \\
& +r_{23} q^{6} \prod_{n=1}^{\infty} \frac{\left(1-q^{4 n}\right)^{20}\left(1-q^{6 n}\right)^{20}}{\left(1-q^{2 n}\right)^{4}\left(1-q^{12 n}\right)^{4}} \\
& +r_{24} q^{12} \prod_{n=1}^{\infty} \frac{\left(1-q^{4 n}\right)^{15}\left(1-q^{6 n}\right)\left(1-q^{12 n}\right)^{19}}{\left(1-q^{2 n}\right)^{3}} \\
& +r_{25} q^{8} \prod_{n=1}^{\infty} \frac{\left(1-q^{2 n}\right)^{20}\left(1-q^{4 n}\right)^{20}}{\left(1-q^{6 n}\right)^{4}\left(1-q^{12 n}\right)^{4}} \\
& +r_{26} q^{12} \prod_{n=1}^{\infty} \frac{\left(1-q^{2 n}\right)^{20}\left(1-q^{6 n}\right)^{20}\left(1-q^{12 n}\right)^{8}}{\left(1-q^{4 n}\right)^{4}} \\
& +r_{27} \prod_{n=1}^{\infty} \frac{\left(1-q^{2 n}\right)^{19}\left(1-q^{4 n}\right)^{13}\left(1-q^{6 n}\right)^{15}}{\left(1-q^{12 n}\right)^{15}} \\
& =\delta\left(b_{1}\right)-\sum_{n=1}^{\infty}\left(c_{1} \sigma_{15}(n)+c_{2} \sigma_{15}\left(\frac{n}{2}\right)+c_{3} \sigma_{15}\left(\frac{n}{3}\right)+c_{4} \sigma_{15}\left(\frac{n}{4}\right)\right. \\
& \left.+c_{6} \sigma_{15}\left(\frac{n}{6}\right)+c_{12} \sigma_{15}\left(\frac{n}{12}\right)\right)+r_{1} f_{1}(n)+\cdots+r_{27} f_{27}(n),
\end{aligned}
$$

where

$$
\delta\left(b_{1}\right)=\left\{\begin{array}{l}
0 \text { if } b_{1} \neq 0 \\
1 \text { if } b_{1}=0
\end{array} .\right.
$$


So

$$
\begin{aligned}
c(n)= & -\left(c_{1} \sigma_{15}(n)+c_{2} \sigma_{15}\left(\frac{n}{2}\right)+c_{3} \sigma_{15}\left(\frac{n}{3}\right)+c_{4} \sigma_{15}\left(\frac{n}{4}\right)\right. \\
& \left.+c_{6} \sigma_{15}\left(\frac{n}{6}\right)+c_{12} \sigma_{15}\left(\frac{n}{12}\right)\right)+r_{1} f_{1}(n)+\cdots+r_{27} f_{27}(n) .
\end{aligned}
$$

Therefore, for $n=1,2, \cdots$,

$$
\begin{gathered}
c(2 n)=-c_{1} \sigma_{15}(2 n)-c_{2} \sigma_{15}(n)-c_{4} \sigma_{15}\left(\frac{n}{2}\right)-\left(65537 c_{3}+c_{6}\right) \sigma_{15}\left(\frac{n}{3}\right) \\
-\left(c_{12}-65536 c_{3}\right) \sigma_{15}\left(\frac{n}{6}\right)+r_{15} f_{15}(2 n)+\cdots+r_{27} f_{27}(2 n), \\
c(2 n-1)=-c_{1} \sigma_{15}(2 n-1)-c_{3} \sigma_{15}\left(\frac{2 n-1}{3}\right)+r_{1} f_{1}(2 n-1)+\cdots+r_{14} f_{14}(2 n-1),
\end{gathered}
$$

since it is easy to see that

$$
\sigma_{k}\left(\frac{2 n}{3}\right)=\left(2^{k}+1\right) \sigma_{k}\left(\frac{n}{3}\right)-2^{k} \sigma_{k}\left(\frac{n}{6}\right)
$$

hence,

$$
\sigma_{15}\left(\frac{2 n}{3}\right)=65537 \sigma_{15}\left(\frac{n}{3}\right)-65536 \sigma_{15}\left(\frac{n}{6}\right)
$$

and, for $n=1,2, \cdots$,

$$
\begin{gathered}
f_{1}(2 n)=\cdots=f_{14}(2 n)=0, \\
f_{15}(2 n-1)=\cdots=f_{27}(2 n-1)=0 .
\end{gathered}
$$

Remark 2 We have found 360 eta quotients, see Table 2, such that, for $n=1,2, \cdots$,

$$
\begin{gathered}
c(2 n)=-c_{1} \sigma_{15}(2 n)-c_{2} \sigma_{15}(n)-c_{4} \sigma_{15}\left(\frac{n}{2}\right)-\left(65537 c_{3}+c_{6}\right) \sigma_{15}\left(\frac{n}{3}\right) \\
-\left(c_{12}-65536 c_{3}\right) \sigma_{15}\left(\frac{n}{6}\right) \\
c(2 n-1)=-c_{1} \sigma_{15}(2 n-1)-c_{3} \sigma_{15}\left(\frac{2 n-1}{3}\right)+r_{1} f_{1}(2 n-1) \\
+\cdots+r_{14} f_{14}(2 n-1) .
\end{gathered}
$$

and 875 eta quotients, such that for $n=1,2, \cdots$,

$$
\begin{gathered}
c(2 n)=-c_{1} \sigma_{15}(2 n)-c_{2} \sigma_{15}(n)-c_{4} \sigma_{15}\left(\frac{n}{2}\right)-c_{6} \sigma_{15}\left(\frac{n}{3}\right) \\
-c_{12} \sigma_{15}\left(\frac{n}{6}\right)+r_{15} f_{15}(2 n)+\cdots+r_{27} f_{27}(2 n), \\
c(2 n-1)=0 .
\end{gathered}
$$

Remark 3 Iff is an eta quotient, then $f(-q)$ is also an eta quotient, so the coefficients of $\frac{1}{2}(f(q)+f(-q))$ are exactly the even coefficients of $f$. In particular, it means that we have obtained all coefficients of some sum of 360 eta quotients. 
Table 2 . The eta quotients whose even coefficients can be explicitly calculated.

\begin{tabular}{|c|c|c|c|c|c|c|c|c|c|c|c|}
\hline No & $a_{2}$ & $a_{4}$ & $a_{6}$ & $a_{12}$ & $C_{1}$ & $c_{2}$ & $c_{3}$ & $c_{4}$ & $c_{6}$ & $C_{12}$ & No \\
\hline 1 & -46 & & 14 & -28 & $\frac{1}{217408}$ & $-\frac{32769}{217408}$ & 0 & $\frac{512}{3397}$ & 0 & 0 & 1 \\
\hline 2 & -45 & & 19 & -29 & $\frac{65}{14348928}$ & $-\frac{64545}{434816}$ & 0 & $\frac{16640}{112101}$ & 0 & 0 & 2 \\
\hline 3 & -44 & & 24 & -30 & $\frac{1}{224202}$ & $-\frac{993}{6794}$ & 0 & $\frac{16384}{112101}$ & 0 & 0 & 3 \\
\hline 4 & -43 & & 29 & -31 & $\frac{21}{4782976}$ & $-\frac{62559}{434816}$ & 0 & $\frac{5376}{37367}$ & 0 & 0 & 4 \\
\hline 5 & -42 & 72 & 34 & -32 & $\frac{31}{7174464}$ & $-\frac{30783}{217408}$ & 0 & $\frac{15872}{112101}$ & 0 & 0 & 5 \\
\hline 6 & -41 & & 39 & -33 & $\frac{61}{14348928}$ & $-\frac{60573}{434816}$ & 0 & $\frac{15616}{112101}$ & 0 & 0 & 6 \\
\hline 7 & -40 & 62 & 44 & -34 & $\frac{5}{1195744}$ & $-\frac{14895}{108704}$ & 0 & $\frac{5120}{37367}$ & 0 & 0 & 7 \\
\hline 8 & -39 & 57 & 49 & -35 & $\frac{59}{14348928}$ & $-\frac{58587}{434816}$ & 0 & $\frac{15104}{112101}$ & 0 & 0 & 8 \\
\hline 9 & -38 & 52 & 54 & -36 & $\frac{29}{7174464}$ & $-\frac{28797}{217408}$ & 0 & $\frac{14848}{112101}$ & 0 & 0 & 9 \\
\hline 10 & -37 & 47 & 59 & -37 & $\frac{19}{4782976}$ & $-\frac{56601}{434816}$ & 0 & $\frac{4864}{37367}$ & 0 & 0 & 10 \\
\hline 11 & -36 & 42 & 64 & -38 & $\frac{7}{1793616}$ & $-\frac{6951}{54352}$ & 0 & $\frac{14336}{112101}$ & 0 & 0 & 11 \\
\hline 12 & -35 & 37 & 69 & -39 & $\frac{5}{1304448}$ & $-\frac{54615}{434816}$ & 0 & $\frac{1280}{10191}$ & 0 & 0 & 12 \\
\hline 13 & -34 & 32 & 74 & -40 & $\frac{9}{2391488}$ & $-\frac{26811}{217408}$ & 0 & $\frac{4608}{37367}$ & 0 & 0 & 13 \\
\hline 14 & -33 & 27 & 79 & -41 & $\frac{53}{14348928}$ & $-\frac{52629}{434816}$ & 0 & $\frac{13568}{112101}$ & 0 & 0 & 14 \\
\hline 15 & -32 & 22 & 84 & -42 & $\frac{13}{3587232}$ & $-\frac{12909}{108704}$ & 0 & $\frac{13312}{112101}$ & 0 & 0 & 15 \\
\hline 16 & -31 & 17 & 89 & -43 & $\frac{6971}{1961319096}$ & $-\frac{2307401}{19811304}$ & $\frac{1}{2187}$ & $\frac{28553216}{245164887}$ & $-\frac{3641}{243}$ & $\frac{32768}{2187}$ & 16 \\
\hline 17 & -37 & 83 & 11 & -25 & $\frac{19}{4782976}$ & $-\frac{56601}{434816}$ & 0 & $\frac{4864}{37367}$ & 0 & 0 & 17 \\
\hline 18 & -36 & 78 & 16 & -26 & $\frac{7}{1793616}$ & $-\frac{6951}{54352}$ & 0 & $\frac{14336}{112101}$ & 0 & 0 & 18 \\
\hline 19 & -35 & 73 & 21 & -27 & $\frac{5}{1304448}$ & $-\frac{54615}{434816}$ & 0 & $\frac{1280}{10191}$ & 0 & 0 & 19 \\
\hline 20 & -34 & 68 & 26 & -28 & $\frac{9}{2391488}$ & $-\frac{26811}{217408}$ & 0 & $\frac{4608}{37367}$ & 0 & 0 & 20 \\
\hline 21 & -33 & & 31 & -29 & $\frac{53}{14348928}$ & $-\frac{52629}{434816}$ & 0 & $\frac{13568}{112101}$ & 0 & 0 & 21 \\
\hline
\end{tabular}




\section{Continued}

\begin{tabular}{|c|c|c|c|c|c|c|c|c|c|c|}
\hline 22 & -3258 & 36 & -30 & $\frac{13}{3587232}$ & $-\frac{12909}{108704}$ & 0 & $\frac{13312}{112101}$ & 0 & 0 & 22 \\
\hline 23 & -3153 & 41 & -31 & $\frac{17}{4782976}$ & $-\frac{50643}{434816}$ & 0 & $\frac{4352}{37367}$ & 0 & 0 & 23 \\
\hline 24 & -3048 & 46 & -32 & $\frac{25}{7174464}$ & $-\frac{24825}{217408}$ & 0 & $\frac{12800}{112101}$ & 0 & 0 & 24 \\
\hline 25 & -2943 & 51 & -33 & $\frac{49}{14348928}$ & $-\frac{48657}{434816}$ & 0 & $\frac{12544}{112101}$ & 0 & 0 & 25 \\
\hline 26 & -2838 & 56 & -34 & $\frac{1}{298936}$ & $-\frac{2979}{27176}$ & 0 & $\frac{4096}{37367}$ & 0 & 0 & 26 \\
\hline 27 & -2733 & 61 & -35 & $\frac{47}{14348928}$ & $-\frac{46671}{434816}$ & 0 & $\frac{12032}{112101}$ & 0 & 0 & 27 \\
\hline 28 & -2628 & 66 & -36 & $\frac{23}{7174464}$ & $-\frac{22839}{217408}$ & 0 & $\frac{11776}{112101}$ & 0 & 0 & 28 \\
\hline 29 & -2523 & 71 & -37 & $\frac{15}{4782976}$ & $-\frac{44685}{434816}$ & 0 & $\frac{3840}{37367}$ & 0 & 0 & 29 \\
\hline 30 & -2418 & 76 & -38 & $\frac{1}{326112}$ & $-\frac{10923}{108704}$ & 0 & $\frac{1024}{10191}$ & 0 & 0 & 30 \\
\hline 31 & -2313 & 81 & -39 & $\frac{653}{217924344}$ & $-\frac{216143}{2201256}$ & $\frac{1}{243}$ & $\frac{2674688}{27240543}$ & $-\frac{3641}{27}$ & $\frac{32768}{243}$ & 31 \\
\hline 32 & -2874 & 8 & -22 & $\frac{1}{298936}$ & $-\frac{2979}{27176}$ & 0 & $\frac{4096}{37367}$ & 0 & 0 & 32 \\
\hline 33 & -2769 & 13 & -23 & $\frac{47}{14348928}$ & $-\frac{46671}{434816}$ & 0 & $\frac{12032}{112101}$ & 0 & 0 & 33 \\
\hline 34 & -2664 & 18 & -24 & $\frac{23}{7174464}$ & $-\frac{22839}{217408}$ & 0 & $\frac{11776}{112101}$ & 0 & 0 & 34 \\
\hline 35 & -2559 & 23 & -25 & $\frac{15}{4782976}$ & $-\frac{44685}{434816}$ & 0 & $\frac{3840}{37367}$ & 0 & 0 & 35 \\
\hline 36 & -2454 & 28 & -26 & $\frac{1}{326112}$ & $-\frac{10923}{108704}$ & 0 & $\frac{1024}{10191}$ & 0 & 0 & 36 \\
\hline 37 & -2349 & 33 & -27 & $\frac{1}{333696}$ & $-\frac{993}{10112}$ & 0 & $\frac{256}{2607}$ & 0 & 0 & 37 \\
\hline 38 & -2244 & 38 & -28 & $\frac{7}{2391488}$ & $-\frac{20853}{217408}$ & 0 & $\frac{3584}{37367}$ & 0 & 0 & 38 \\
\hline 39 & -2139 & 43 & -29 & $\frac{41}{14348928}$ & $-\frac{40713}{434816}$ & 0 & $\frac{10496}{112101}$ & 0 & 0 & 39 \\
\hline 40 & -2034 & 48 & -30 & $\frac{5}{1793616}$ & $-\frac{4965}{54352}$ & 0 & $\frac{10240}{112101}$ & 0 & 0 & 40 \\
\hline 41 & -1929 & 53 & -31 & $\frac{13}{4782976}$ & $-\frac{38727}{434816}$ & 0 & $\frac{3328}{37367}$ & 0 & 0 & 41 \\
\hline 42 & -1824 & 58 & -32 & $\frac{19}{7174464}$ & $-\frac{18867}{217408}$ & 0 & $\frac{9728}{112101}$ & 0 & 0 & 42 \\
\hline 43 & -1719 & 63 & -33 & $\frac{37}{14348928}$ & $-\frac{36741}{434816}$ & 0 & $\frac{9472}{112101}$ & 0 & 0 & 43 \\
\hline
\end{tabular}




\section{Continued}

\begin{tabular}{|c|c|c|c|c|c|c|c|c|c|c|}
\hline 44 & -1614 & 68 & -34 & $\frac{3}{1195744}$ & $-\frac{8937}{108704}$ & 0 & $\frac{3072}{37367}$ & 0 & 0 & 44 \\
\hline 45 & -159 & 73 & -35 & $\frac{59}{24213816}$ & $-\frac{19529}{244584}$ & $\frac{1}{27}$ & $\frac{241664}{3026727}$ & $-\frac{3641}{3}$ & $\frac{32768}{27}$ & 45 \\
\hline 46 & -1965 & 5 & -19 & $\frac{13}{4782976}$ & $-\frac{38727}{434816}$ & 0 & $\frac{3328}{37367}$ & 0 & 0 & 46 \\
\hline 47 & -1860 & 10 & -20 & $\frac{19}{7174464}$ & $-\frac{18867}{217408}$ & 0 & $\frac{9728}{112101}$ & 0 & 0 & 47 \\
\hline 48 & -1755 & 15 & -21 & $\frac{37}{14348928}$ & $-\frac{36741}{434816}$ & 0 & $\frac{9472}{112101}$ & 0 & 0 & 48 \\
\hline 49 & -1650 & 20 & -22 & $\frac{3}{1195744}$ & $-\frac{8937}{108704}$ & 0 & $\frac{3072}{37367}$ & 0 & 0 & 49 \\
\hline 50 & -1545 & 25 & -23 & $\frac{35}{14348928}$ & $-\frac{34755}{434816}$ & 0 & $\frac{8960}{112101}$ & 0 & 0 & 50 \\
\hline 51 & -1440 & 30 & -24 & $\frac{17}{7174464}$ & $-\frac{16881}{217408}$ & 0 & $\frac{8704}{112101}$ & 0 & 0 & 51 \\
\hline 52 & -1335 & 35 & -25 & $\frac{1}{434816}$ & $-\frac{32769}{434816}$ & 0 & $\frac{256}{3397}$ & 0 & 0 & 52 \\
\hline 53 & -1230 & 40 & -26 & $\frac{1}{448404}$ & $-\frac{993}{13588}$ & 0 & $\frac{8192}{112101}$ & 0 & 0 & 53 \\
\hline 54 & -1125 & 45 & -27 & $\frac{31}{14348928}$ & $-\frac{30783}{434816}$ & 0 & $\frac{7936}{112101}$ & 0 & 0 & 54 \\
\hline 55 & -1020 & 50 & -28 & $\frac{5}{2391488}$ & $-\frac{14895}{217408}$ & 0 & $\frac{2560}{37367}$ & 0 & 0 & 55 \\
\hline 56 & $\begin{array}{ll}-9 & 15\end{array}$ & 55 & -29 & $\frac{29}{14348928}$ & $-\frac{28797}{434816}$ & 0 & $\frac{7424}{112101}$ & 0 & 0 & 56 \\
\hline 57 & $\begin{array}{ll}-8 & 10\end{array}$ & 60 & -30 & $\frac{7}{3587232}$ & $-\frac{6951}{108704}$ & 0 & $\frac{7168}{112101}$ & 0 & 0 & 57 \\
\hline 58 & $\begin{array}{ll}-7 & 5\end{array}$ & 65 & -31 & $\frac{5}{2690424}$ & $-\frac{1655}{27176}$ & $\frac{1}{3}$ & $\frac{20480}{336303}$ & -10923 & $\frac{32768}{3}$ & 58 \\
\hline 59 & -1056 & 2 & -16 & $\frac{5}{2391488}$ & $-\frac{14895}{217408}$ & 0 & $\frac{2560}{37367}$ & 0 & 0 & 59 \\
\hline 60 & $\begin{array}{ll}-9 & 51\end{array}$ & 7 & -17 & $\frac{29}{14348928}$ & $-\frac{28797}{434816}$ & 0 & $\frac{7424}{112101}$ & 0 & 0 & 60 \\
\hline 61 & -846 & 12 & -18 & $\frac{7}{3587232}$ & $-\frac{6951}{108704}$ & 0 & $\frac{7168}{112101}$ & 0 & 0 & 61 \\
\hline 62 & $\begin{array}{ll}-7 & 41\end{array}$ & 17 & -19 & $\frac{9}{4782976}$ & $-\frac{26811}{434816}$ & 0 & $\frac{2304}{37367}$ & 0 & 0 & 62 \\
\hline 63 & -636 & 22 & -20 & $\frac{13}{7174464}$ & $-\frac{12909}{217408}$ & 0 & $\frac{6656}{112101}$ & 0 & 0 & 63 \\
\hline 64 & $-5 \quad 31$ & 27 & -21 & $\frac{25}{14348918}$ & $-\frac{24825}{434816}$ & 0 & $\frac{6400}{112101}$ & 0 & 0 & 64 \\
\hline 65 & $\begin{array}{ll}-4 & 26\end{array}$ & 32 & -22 & $\frac{1}{597872}$ & $-\frac{2979}{54352}$ & 0 & $\frac{2048}{37367}$ & 0 & 0 & 65 \\
\hline
\end{tabular}




\section{Continued}

\begin{tabular}{|c|c|c|c|c|c|c|c|c|c|c|c|}
\hline 66 & -3 & 21 & 37 & -23 & $\frac{23}{14348928}$ & $-\frac{22839}{434816}$ & 0 & $\frac{5888}{112101}$ & 0 & 0 & 66 \\
\hline 67 & -2 & 16 & 42 & -24 & $\frac{1}{652224}$ & $-\frac{10923}{217408}$ & 0 & $\frac{512}{10191}$ & 0 & 0 & 67 \\
\hline 68 & -1 & 11 & 47 & -25 & $\frac{7}{4782976}$ & $-\frac{20853}{434816}$ & 0 & $\frac{1792}{37367}$ & 0 & 0 & 68 \\
\hline 69 & 0 & 6 & 52 & -26 & $\frac{5}{3587232}$ & $-\frac{4965}{108704}$ & 0 & $\frac{5120}{112101}$ & 0 & 0 & 69 \\
\hline 70 & 1 & 1 & 57 & -27 & $\frac{1}{896808}$ & $-\frac{993}{27176}$ & 3 & $\frac{4096}{112101}$ & -98307 & 98304 & 70 \\
\hline 71 & -1 & 47 & -1 & -13 & $\frac{7}{4782976}$ & $-\frac{20853}{434816}$ & 0 & $\frac{1792}{37367}$ & 0 & 0 & 71 \\
\hline 72 & 0 & 42 & 4 & -14 & $\frac{5}{3587232}$ & $-\frac{4965}{108704}$ & 0 & $\frac{5120}{112101}$ & 0 & 0 & 72 \\
\hline 73 & 1 & 37 & 9 & -15 & $\frac{19}{14348928}$ & $-\frac{18867}{434816}$ & 0 & $\frac{4864}{112101}$ & 0 & 0 & 73 \\
\hline 74 & 2 & 32 & 14 & -16 & $\frac{3}{2391488}$ & $-\frac{8937}{217408}$ & 0 & $\frac{1536}{37367}$ & 0 & 0 & 74 \\
\hline 75 & 3 & 27 & 19 & -17 & $\frac{17}{14348928}$ & $-\frac{16881}{434816}$ & 0 & $\frac{4352}{112101}$ & 0 & 0 & 75 \\
\hline 76 & 4 & 22 & 24 & -18 & $\frac{1}{896808}$ & $-\frac{993}{27176}$ & 0 & $\frac{4096}{112101}$ & 0 & 0 & 76 \\
\hline 77 & 5 & 17 & 29 & -19 & $\frac{5}{4782976}$ & $-\frac{14895}{434816}$ & 0 & $\frac{1280}{37367}$ & 0 & 0 & 77 \\
\hline 78 & 6 & 12 & 34 & -20 & $\frac{7}{7174464}$ & $-\frac{6951}{217408}$ & 0 & $\frac{3584}{112101}$ & 0 & 0 & 78 \\
\hline 79 & 7 & 7 & 39 & -21 & $\frac{13}{14348928}$ & $-\frac{12909}{434816}$ & 0 & $\frac{3328}{112101}$ & 0 & 0 & 79 \\
\hline 80 & 8 & 2 & 44 & -22 & $\frac{1}{1195744}$ & $-\frac{2979}{108704}$ & 0 & $\frac{1024}{37367}$ & 0 & 0 & 80 \\
\hline 81 & 9 & -3 & 49 & -23 & $-\frac{1}{896808}$ & $\frac{993}{27176}$ & 27 & $-\frac{4096}{112101}$ & -884763 & 884736 & 81 \\
\hline 82 & 8 & 38 & -4 & -10 & $\frac{1}{1195744}$ & $-\frac{2979}{108704}$ & 0 & $\frac{1024}{37367}$ & 0 & 0 & 82 \\
\hline 83 & 9 & 33 & 1 & -11 & $\frac{1}{1304448}$ & $-\frac{10923}{434816}$ & 0 & $\frac{256}{10191}$ & 0 & 0 & 83 \\
\hline 84 & 10 & 28 & 6 & -12 & $\frac{5}{7174464}$ & $-\frac{4965}{217408}$ & 0 & $\frac{2560}{112101}$ & 0 & 0 & 84 \\
\hline 85 & 11 & 23 & 11 & -13 & $\frac{3}{4782976}$ & $-\frac{8937}{434816}$ & 0 & $\frac{768}{37367}$ & 0 & 0 & 85 \\
\hline 86 & 12 & 18 & 16 & -14 & $\frac{1}{1793616}$ & $-\frac{993}{54352}$ & 0 & $\frac{2048}{112101}$ & 0 & 0 & 86 \\
\hline 87 & 13 & 13 & 21 & -15 & $\frac{7}{14348928}$ & $-\frac{6951}{434816}$ & 0 & $\frac{1792}{112101}$ & 0 & 0 & 87 \\
\hline
\end{tabular}




\section{Continued}

\begin{tabular}{|c|c|c|c|c|c|c|c|c|c|c|c|}
\hline 88 & 14 & 8 & 26 & -16 & $\frac{1}{2391488}$ & $-\frac{2979}{217408}$ & 0 & $\frac{512}{37367}$ & 0 & 0 & 88 \\
\hline 89 & 15 & 3 & 31 & -17 & $\frac{5}{14348928}$ & $-\frac{4965}{434816}$ & 0 & $\frac{1280}{112101}$ & 0 & 0 & 89 \\
\hline 90 & 16 & -2 & 36 & -18 & $\frac{1}{3587232}$ & $-\frac{993}{108704}$ & 0 & $\frac{1024}{112101}$ & 0 & 0 & 90 \\
\hline 91 & 17 & -7 & 41 & -19 & $-\frac{5}{298936}$ & $\frac{14895}{27176}$ & 243 & $-\frac{20480}{37367}$ & -7962867 & 7962624 & 91 \\
\hline 92 & 17 & 29 & -7 & -7 & $\frac{1}{4782976}$ & $-\frac{2979}{434816}$ & 0 & $\frac{256}{37367}$ & 0 & 0 & 92 \\
\hline 93 & 18 & 24 & -2 & -8 & $\frac{1}{7174464}$ & $-\frac{993}{217408}$ & 0 & $\frac{512}{112101}$ & 0 & 0 & 93 \\
\hline 94 & 19 & 19 & 3 & -9 & $\frac{1}{14348928}$ & $-\frac{993}{434816}$ & 0 & $\frac{256}{112101}$ & 0 & 0 & 94 \\
\hline 95 & 21 & 9 & 13 & -11 & $-\frac{1}{14348928}$ & $\frac{993}{434816}$ & 0 & $-\frac{256}{112101}$ & 0 & 0 & 95 \\
\hline 96 & 22 & 4 & 18 & -12 & $-\frac{1}{7174464}$ & $\frac{993}{217408}$ & 0 & $-\frac{512}{112101}$ & 0 & 0 & 96 \\
\hline 97 & 23 & -1 & 23 & -13 & $-\frac{1}{4782976}$ & $\frac{2979}{434816}$ & 0 & $-\frac{256}{37367}$ & 0 & 0 & 97 \\
\hline 98 & 24 & -6 & 28 & -14 & $-\frac{1}{3587232}$ & $\frac{993}{108704}$ & 0 & $-\frac{1024}{112101}$ & 0 & 0 & 98 \\
\hline 99 & 25 & -11 & 33 & -15 & $-\frac{137}{896808}$ & $\frac{136041}{27176}$ & 2187 & $-\frac{561152}{112101}$ & -71665803 & 71663616 & 99 \\
\hline 100 & 26 & 20 & -10 & -4 & $-\frac{1}{2391488}$ & $\frac{2979}{217408}$ & 0 & $-\frac{512}{37367}$ & 0 & 0 & 100 \\
\hline 101 & 27 & 15 & -5 & -5 & $-\frac{7}{14348928}$ & $\frac{6951}{434816}$ & 0 & $-\frac{1792}{112101}$ & 0 & 0 & 101 \\
\hline 102 & 28 & 10 & 0 & -6 & $-\frac{1}{1793616}$ & $\frac{993}{54352}$ & 0 & $-\frac{2048}{112101}$ & 0 & 0 & 102 \\
\hline 103 & 29 & 5 & 5 & -7 & $-\frac{3}{4782976}$ & $\frac{8937}{434816}$ & 0 & $-\frac{768}{37367}$ & 0 & 0 & 103 \\
\hline 104 & 30 & 0 & 10 & -8 & $-\frac{5}{7174464}$ & $\frac{4965}{217408}$ & 0 & $-\frac{2560}{112101}$ & 0 & 0 & 104 \\
\hline 105 & 31 & -5 & 15 & -9 & $-\frac{1}{1304448}$ & $\frac{10923}{434816}$ & 0 & $-\frac{256}{10191}$ & 0 & 0 & 105 \\
\hline 106 & 32 & -10 & 20 & -10 & $-\frac{1}{1195744}$ & $\frac{2979}{108704}$ & 0 & $-\frac{1024}{37367}$ & 0 & 0 & 106 \\
\hline 107 & 33 & -15 & 25 & -11 & $-\frac{1231}{896808}$ & $\frac{1222383}{27176}$ & 19683 & $-\frac{5042176}{112101}$ & -644992227 & 644972544 & 107 \\
\hline 108 & 35 & 11 & -13 & -1 & $-\frac{5}{4782976}$ & $\frac{14895}{434816}$ & 0 & $-\frac{1280}{37367}$ & 0 & 0 & 108 \\
\hline 109 & 36 & 6 & -8 & 2 & $-\frac{1}{896808}$ & $\frac{993}{27176}$ & 0 & $-\frac{4096}{112101}$ & 0 & 0 & 109 \\
\hline
\end{tabular}




\section{Continued}

\begin{tabular}{|c|c|c|c|c|c|c|c|c|c|c|c|}
\hline 110 & 37 & 1 & -3 & -3 & $-\frac{17}{14348928}$ & $\frac{16881}{434816}$ & 0 & $-\frac{4352}{112101}$ & 0 & 0 & 110 \\
\hline 111 & 38 & -4 & 2 & -4 & $-\frac{3}{2391488}$ & $\frac{8937}{217408}$ & 0 & $-\frac{1536}{37367}$ & 0 & 0 & 111 \\
\hline 112 & 39 & -9 & 7 & -5 & $-\frac{19}{14348928}$ & $\frac{18867}{434816}$ & 0 & $-\frac{4864}{112101}$ & 0 & 0 & 112 \\
\hline 113 & 40 & -14 & 12 & -6 & $-\frac{5}{3587232}$ & $\frac{4965}{108704}$ & 0 & $-\frac{5120}{112101}$ & 0 & 0 & 113 \\
\hline 114 & 41 & -19 & 17 & -7 & $-\frac{3691}{298936}$ & $\frac{10995489}{27176}$ & 177147 & $-\frac{15118336}{37367}$ & -5804930043 & 5804752896 & 114 \\
\hline 115 & 44 & 2 & -16 & 2 & $-\frac{1}{597872}$ & $\frac{2979}{54352}$ & 0 & $-\frac{2048}{37367}$ & 0 & 0 & 115 \\
\hline 116 & 45 & -3 & -11 & 1 & $-\frac{25}{14348918}$ & $\frac{24825}{434816}$ & 0 & $-\frac{6400}{112101}$ & 0 & 0 & 116 \\
\hline 117 & 46 & -8 & -6 & 0 & $-\frac{13}{7174464}$ & $\frac{12909}{217408}$ & 0 & $-\frac{6656}{112101}$ & 0 & 0 & 117 \\
\hline 118 & 47 & -13 & -1 & -1 & $-\frac{9}{4782976}$ & $\frac{26811}{434816}$ & 0 & $-\frac{2304}{37367}$ & 0 & 0 & 118 \\
\hline 119 & 48 & -18 & 4 & -2 & $-\frac{7}{3587232}$ & $\frac{6951}{108704}$ & 0 & $-\frac{7168}{112101}$ & 0 & 0 & 119 \\
\hline 120 & 49 & -23 & 9 & -3 & $-\frac{99647}{896808}$ & $\frac{98949471}{27176}$ & 1594323 & $-\frac{408154112}{112101}$ & -52244370387 & 52242776064 & 120 \\
\hline 121 & 53 & -7 & -19 & 5 & $-\frac{1}{434816}$ & $\frac{32769}{434816}$ & 0 & $-\frac{256}{3397}$ & 0 & 0 & 121 \\
\hline 122 & 54 & -12 & -14 & 4 & $-\frac{17}{7174464}$ & $\frac{16881}{217408}$ & 0 & $-\frac{8704}{112101}$ & 0 & 0 & 122 \\
\hline 123 & 55 & -17 & -9 & 3 & $-\frac{35}{14348928}$ & $\frac{34755}{434816}$ & 0 & $-\frac{8960}{112101}$ & 0 & 0 & 123 \\
\hline 124 & 56 & -22 & -4 & 2 & $-\frac{3}{1195744}$ & $\frac{8937}{108704}$ & 0 & $-\frac{3072}{37367}$ & 0 & 0 & 124 \\
\hline 125 & 57 & -27 & 1 & 1 & $-\frac{896809}{896808}$ & $\frac{890531337}{27176}$ & 14348907 & $-\frac{3673329664}{112101}$ & -470199333483 & 470184984576 & 125 \\
\hline 126 & 62 & -16 & -22 & 8 & $-\frac{7}{2391488}$ & $\frac{20853}{217408}$ & 0 & $-\frac{3584}{37367}$ & 0 & 0 & 126 \\
\hline 127 & 63 & -21 & -17 & 7 & $-\frac{1}{333696}$ & $\frac{993}{10112}$ & 0 & $-\frac{256}{2607}$ & 0 & 0 & 127 \\
\hline 128 & 64 & -26 & -12 & 6 & $-\frac{1}{326112}$ & $\frac{10923}{108704}$ & 0 & $-\frac{1024}{10191}$ & 0 & 0 & 128 \\
\hline 129 & 65 & -31 & -7 & 5 & $-\frac{2690421}{298936}$ & $\frac{8014764159}{27176}$ & 129140163 & $-\frac{11019964416}{37367}$ & -4231794001347 & 4231664861184 & 129 \\
\hline 130 & 71 & -25 & -25 & 11 & $-\frac{17}{4782976}$ & $\frac{50643}{434816}$ & 0 & $-\frac{4352}{37367}$ & 0 & 0 & 130 \\
\hline 131 & 72 & -30 & -20 & 10 & $\frac{13}{3587232}$ & $-\frac{12909}{108704}$ & 0 & $\frac{13312}{112101}$ & 0 & 0 & 131 \\
\hline
\end{tabular}




\section{Continued}

\begin{tabular}{|c|c|c|c|c|c|c|c|c|}
\hline $13273-35$ & -159 & $\frac{72641345}{896808}$ & $\frac{72132855585}{27176}$ & 1162261467 & $-\frac{297538949120}{112101}$ & -38086146012123 & 38084983750656 & 132 \\
\hline $13380-34$ & -2814 & $-\frac{5}{1195744}$ & $\frac{14895}{108704}$ & 0 & $-\frac{5120}{37367}$ & 0 & 0 & 133 \\
\hline $13481-39$ & -2313 & $-\frac{653772079}{896808}$ & $\frac{649195674447}{27176}$ & 10460353203 & $-\frac{2677850435584}{112101}$ & -342775314109107 & 342764853755904 & 134 \\
\hline $13589-43$ & -3117 & $-\frac{1961316227}{298936}$ & $\frac{5842761040233}{27176}$ & 94143178827 & $-\frac{8033551265792}{37367}$ & -3084977826981963 & 3084883683803136 & 135 \\
\hline $136-4284$ & $10-20$ & $\frac{1}{14348928}$ & $-\frac{993}{434816}$ & 0 & $\frac{256}{112101}$ & 0 & 0 & 136 \\
\hline $137-4179$ & $15-21$ & $\frac{1}{14348928}$ & $-\frac{993}{434816}$ & 0 & $\frac{256}{112101}$ & 0 & 0 & 137 \\
\hline $138-4074$ & $20-22$ & $\frac{1}{14348928}$ & $-\frac{993}{434816}$ & 0 & $\frac{256}{112101}$ & 0 & 0 & 138 \\
\hline $139-3969$ & $25-23$ & $\frac{1}{14348928}$ & $-\frac{993}{434816}$ & 0 & $\frac{256}{112101}$ & 0 & 0 & 139 \\
\hline $140-3864$ & $30-24$ & $\frac{1}{14348928}$ & $-\frac{993}{434816}$ & 0 & $\frac{256}{112101}$ & 0 & 0 & 140 \\
\hline $141-3759$ & $35-25$ & $\frac{1}{14348928}$ & $-\frac{993}{434816}$ & 0 & $\frac{256}{112101}$ & 0 & 0 & 141 \\
\hline $142-3654$ & $40-26$ & $\frac{1}{14348928}$ & $-\frac{993}{434816}$ & 0 & $\frac{256}{112101}$ & 0 & 0 & 142 \\
\hline $143-3549$ & $45-27$ & $\frac{1}{14348928}$ & $-\frac{993}{434816}$ & 0 & $\frac{256}{112101}$ & 0 & 0 & 143 \\
\hline $144-3444$ & $50-28$ & $\frac{1}{14348928}$ & $-\frac{993}{434816}$ & 0 & $\frac{256}{112101}$ & 0 & 0 & 144 \\
\hline $145-3339$ & $55-29$ & $\frac{1}{14348928}$ & $-\frac{993}{434816}$ & 0 & $\frac{256}{112101}$ & 0 & 0 & 145 \\
\hline $146-3234$ & $60-30$ & $\frac{1}{14348928}$ & $-\frac{993}{434816}$ & 0 & $\frac{256}{112101}$ & 0 & 0 & 146 \\
\hline $147-3129$ & $65-31$ & $\frac{1}{14348928}$ & $\frac{993}{434816}$ & 0 & $\frac{256}{112101}$ & 0 & 0 & 147 \\
\hline $148-3024$ & $70-32$ & $\frac{1}{14348928}$ & $-\frac{993}{434816}$ & 0 & $\frac{256}{112101}$ & 0 & 0 & 148 \\
\hline $149-2919$ & $75-33$ & $\frac{1}{14348928}$ & $-\frac{993}{434816}$ & 0 & $\frac{256}{112101}$ & 0 & 0 & 149 \\
\hline $150-2814$ & $80-34$ & $\frac{547}{7845276384}$ & $-\frac{181057}{79245216}$ & $-\frac{1}{2187}$ & $\frac{560128}{245164887}$ & $\frac{3641}{243}$ & $-\frac{32768}{2187}$ & 150 \\
\hline $151-3375$ & $\begin{array}{ll}7 & -17\end{array}$ & $\frac{1}{14348928}$ & $\frac{993}{434816}$ & 0 & $\frac{256}{112101}$ & 0 & 0 & 151 \\
\hline $152-3270$ & $12-18$ & $\frac{1}{14348928}$ & $-\frac{993}{434816}$ & 0 & $\frac{256}{112101}$ & 0 & 0 & 152 \\
\hline $153-3165$ & $17-19$ & $\frac{1}{14348928}$ & $-\frac{993}{434816}$ & 0 & $\frac{256}{112101}$ & 0 & 0 & 153 \\
\hline
\end{tabular}




\section{Continued}

\begin{tabular}{|c|c|c|c|c|c|c|c|c|}
\hline $154-3060$ & $22-20$ & $\frac{1}{14348928}$ & $-\frac{993}{434816}$ & 0 & $\frac{256}{112101}$ & 0 & 0 & 154 \\
\hline $155-2955$ & $27-21$ & $\frac{1}{14348928}$ & $-\frac{993}{434816}$ & 0 & $\frac{256}{112101}$ & 0 & 0 & 155 \\
\hline $156-2850$ & $32-22$ & $\frac{1}{14348928}$ & $-\frac{993}{434816}$ & 0 & $\frac{256}{112101}$ & 0 & 0 & 156 \\
\hline $157-2745$ & $37-23$ & $\frac{1}{14348928}$ & $-\frac{993}{434816}$ & 0 & $\frac{256}{112101}$ & 0 & 0 & 157 \\
\hline $158-2640$ & $42-24$ & $\frac{1}{14348928}$ & $-\frac{993}{434816}$ & 0 & $\frac{256}{112101}$ & 0 & 0 & 158 \\
\hline $159-2535$ & $47-25$ & $\frac{1}{14348928}$ & $-\frac{993}{434816}$ & 0 & $\frac{256}{112101}$ & 0 & 0 & 159 \\
\hline $160-2430$ & $52-26$ & $\frac{1}{14348928}$ & $-\frac{993}{434816}$ & 0 & $\frac{256}{112101}$ & 0 & 0 & 160 \\
\hline $161-2325$ & $57-27$ & $\frac{1}{14348928}$ & $-\frac{993}{434816}$ & 0 & $\frac{256}{112101}$ & 0 & 0 & 161 \\
\hline $162-2220$ & $62-28$ & $\frac{1}{14348928}$ & $-\frac{993}{434816}$ & 0 & $\frac{256}{112101}$ & 0 & 0 & 162 \\
\hline $163-2115$ & $67-29$ & $\frac{1}{14348928}$ & $-\frac{993}{434816}$ & 0 & $\frac{256}{112101}$ & 0 & 0 & 163 \\
\hline $164-2010$ & $72-30$ & $\frac{61}{871697376}$ & $-\frac{20191}{8805024}$ & $\frac{1}{243}$ & $\frac{62464}{27240543}$ & $\frac{3641}{27}$ & $-\frac{32768}{243}$ & 164 \\
\hline $165-2466$ & $\begin{array}{ll}4 & -14\end{array}$ & $\frac{1}{14348928}$ & $-\frac{993}{434816}$ & 0 & $\frac{256}{112101}$ & 0 & 0 & 165 \\
\hline $166-2361$ & $\begin{array}{ll}9 & -15\end{array}$ & $\frac{1}{14348928}$ & $-\frac{993}{434816}$ & 0 & $\frac{256}{112101}$ & 0 & 0 & 166 \\
\hline $167-2256$ & $14-16$ & $\frac{1}{14348928}$ & $-\frac{993}{434816}$ & 0 & $\frac{256}{112101}$ & 0 & 0 & 167 \\
\hline $168-2151$ & $19-17$ & $\frac{1}{14348928}$ & $-\frac{993}{434816}$ & 0 & $\frac{256}{112101}$ & 0 & 0 & 168 \\
\hline $169-2046$ & $24-18$ & $\frac{1}{14348928}$ & $-\frac{993}{434816}$ & 0 & $\frac{256}{112101}$ & 0 & 0 & 169 \\
\hline $170-1941$ & $29-19$ & $\frac{1}{14348928}$ & $-\frac{993}{434816}$ & 0 & $\frac{256}{112101}$ & 0 & 0 & 170 \\
\hline $171-1836$ & $34-20$ & $\frac{1}{14348928}$ & $\frac{993}{434816}$ & 0 & $\frac{256}{112101}$ & 0 & 0 & 171 \\
\hline $172-1731$ & $39-21$ & $\frac{1}{14348928}$ & $-\frac{993}{434816}$ & 0 & $\frac{256}{112101}$ & 0 & 0 & 172 \\
\hline $173-1626$ & $44-22$ & $\frac{1}{14348928}$ & $-\frac{993}{434816}$ & 0 & $\frac{256}{112101}$ & 0 & 0 & 173 \\
\hline $174-1521$ & $49-23$ & $\frac{1}{14348928}$ & $\frac{993}{434816}$ & 0 & $\frac{256}{112101}$ & 0 & 0 & 174 \\
\hline $175-1416$ & $54-24$ & $\frac{1}{14348928}$ & $-\frac{993}{434816}$ & 0 & $\frac{256}{112101}$ & 0 & 0 & 175 \\
\hline
\end{tabular}




\section{Continued}

\begin{tabular}{|c|c|c|c|c|c|c|c|c|}
\hline $176-1311$ & $59-25$ & $\frac{1}{14348928}$ & $-\frac{993}{434816}$ & 0 & $\frac{256}{112101}$ & 0 & 0 & 176 \\
\hline $177-126$ & $64-26$ & $\frac{7}{96855264}$ & $-\frac{2317}{978336}$ & $-\frac{1}{27}$ & $\frac{7168}{3026727}$ & $\frac{3641}{3}$ & $-\frac{32768}{27}$ & 177 \\
\hline $178-1557$ & $1-11$ & $\frac{1}{14348928}$ & $-\frac{993}{434816}$ & 0 & $\frac{256}{112101}$ & 0 & 0 & 178 \\
\hline $179-1452$ & $\begin{array}{ll}6 & -12\end{array}$ & $\frac{1}{14348928}$ & $-\frac{993}{434816}$ & 0 & $\frac{256}{112101}$ & 0 & 0 & 179 \\
\hline $180-1347$ & $11-13$ & $\frac{1}{14348928}$ & $-\frac{993}{434816}$ & 0 & $\frac{256}{112101}$ & 0 & 0 & 180 \\
\hline $181-1242$ & $16-14$ & $\frac{1}{14348928}$ & $-\frac{993}{434816}$ & 0 & $\frac{256}{112101}$ & 0 & 0 & 181 \\
\hline $182-1137$ & $21-15$ & $\frac{1}{14348928}$ & $-\frac{993}{434816}$ & 0 & $\frac{256}{112101}$ & 0 & 0 & 182 \\
\hline $183-1032$ & $26-16$ & $\frac{1}{14348928}$ & $-\frac{993}{434816}$ & 0 & $\frac{256}{112101}$ & 0 & 0 & 183 \\
\hline $184-927$ & $31-17$ & $\frac{1}{14348928}$ & $-\frac{993}{434816}$ & 0 & $\frac{256}{112101}$ & 0 & 0 & 184 \\
\hline $185-822$ & $36-18$ & $\frac{1}{14348928}$ & $-\frac{993}{434816}$ & 0 & $\frac{256}{112101}$ & 0 & 0 & 185 \\
\hline $186-7 \quad 17$ & $41-19$ & $\frac{1}{14348928}$ & $-\frac{993}{434816}$ & 0 & $\frac{256}{112101}$ & 0 & 0 & 186 \\
\hline $187-6 \quad 12$ & $46-20$ & $\frac{1}{14348928}$ & $-\frac{993}{434816}$ & 0 & $\frac{256}{112101}$ & 0 & 0 & 187 \\
\hline $188-5 \quad 7$ & $51-21$ & $\frac{1}{14348928}$ & $-\frac{993}{434816}$ & 0 & $\frac{256}{112101}$ & 0 & 0 & 188 \\
\hline $189-4 \quad 2$ & $56-22$ & $\frac{1}{10761696}$ & $-\frac{331}{108704}$ & $-\frac{1}{3}$ & $\frac{1024}{336303}$ & 10923 & $-\frac{32768}{3}$ & 189 \\
\hline $190-6 \quad 48$ & $\begin{array}{ll}-2 & -8\end{array}$ & $\frac{1}{14348928}$ & $-\frac{993}{434816}$ & 0 & $\frac{256}{112101}$ & 0 & 0 & 190 \\
\hline $191-543$ & $\begin{array}{ll}3 & -9\end{array}$ & $\frac{1}{14348928}$ & $-\frac{993}{434816}$ & 0 & $\frac{256}{112101}$ & 0 & 0 & 191 \\
\hline $192-438$ & $\begin{array}{ll}8 & -9\end{array}$ & $\frac{1}{14348928}$ & $-\frac{993}{434816}$ & 0 & $\frac{256}{112101}$ & 0 & 0 & 192 \\
\hline $193-333$ & $13-11$ & $\frac{1}{14348928}$ & $-\frac{993}{434816}$ & 0 & $\frac{256}{112101}$ & 0 & 0 & 193 \\
\hline $194-228$ & $18-12$ & $\frac{1}{14348928}$ & $-\frac{993}{434816}$ & 0 & $\frac{256}{112101}$ & 0 & 0 & 194 \\
\hline $195-123$ & $23-13$ & $\frac{1}{14348928}$ & $-\frac{993}{434816}$ & 0 & $\frac{256}{112101}$ & 0 & 0 & 195 \\
\hline $\begin{array}{lll}196 & 0 & 18\end{array}$ & $28-14$ & $\frac{1}{14348928}$ & $\frac{993}{434816}$ & 0 & $\frac{256}{112101}$ & 0 & 0 & 196 \\
\hline $\begin{array}{lll}197 \quad 1 & 13\end{array}$ & $33-15$ & $\frac{1}{14348928}$ & $-\frac{993}{434816}$ & 0 & $\frac{256}{112101}$ & 0 & 0 & 197 \\
\hline
\end{tabular}




\section{Continued}

\begin{tabular}{|c|c|c|c|c|c|c|c|c|c|c|c|}
\hline 198 & 2 & 8 & 38 & -16 & $\frac{1}{14348928}$ & $-\frac{993}{434816}$ & 0 & $\frac{256}{112101}$ & 0 & 0 & 198 \\
\hline 199 & 3 & 3 & 43 & -17 & $\frac{1}{14348928}$ & $-\frac{993}{434816}$ & 0 & $\frac{256}{112101}$ & 0 & 0 & 199 \\
\hline 200 & 4 & -2 & 48 & -18 & $\frac{1}{3587232}$ & $-\frac{993}{108704}$ & -3 & $\frac{1024}{112101}$ & 98307 & -98304 & 200 \\
\hline 201 & 3 & 39 & -5 & -5 & $\frac{1}{14348928}$ & $-\frac{993}{434816}$ & 0 & $\frac{256}{112101}$ & 0 & 0 & 201 \\
\hline 202 & 4 & 34 & 0 & -6 & $\frac{1}{14348928}$ & $-\frac{993}{434816}$ & 0 & $\frac{256}{112101}$ & 0 & 0 & 202 \\
\hline 203 & 5 & 29 & 5 & -7 & $\frac{1}{14348928}$ & $-\frac{993}{434816}$ & 0 & $\frac{256}{112101}$ & 0 & 0 & 203 \\
\hline 204 & 6 & 24 & 10 & -8 & $\frac{1}{14348928}$ & $-\frac{993}{434816}$ & 0 & $\frac{256}{112101}$ & 0 & 0 & 204 \\
\hline 205 & 7 & 19 & 15 & -9 & $\frac{1}{14348928}$ & $-\frac{993}{434816}$ & 0 & $\frac{256}{112101}$ & 0 & 0 & 205 \\
\hline 206 & 8 & 14 & 20 & -10 & $\frac{1}{14348928}$ & $-\frac{993}{434816}$ & 0 & $\frac{256}{112101}$ & 0 & 0 & 206 \\
\hline 207 & 9 & 9 & 25 & -11 & $\frac{1}{14348928}$ & $-\frac{993}{434816}$ & 0 & $\frac{256}{112101}$ & 0 & 0 & 207 \\
\hline 208 & 10 & 4 & 30 & -12 & $\frac{1}{14348928}$ & $-\frac{993}{434816}$ & 0 & $\frac{256}{112101}$ & 0 & 0 & 208 \\
\hline 209 & 11 & -1 & 35 & -13 & $\frac{1}{14348928}$ & $-\frac{993}{434816}$ & 0 & $\frac{256}{112101}$ & 0 & 0 & 209 \\
\hline 210 & 12 & -6 & 40 & -14 & $\frac{7}{3587232}$ & $-\frac{6951}{108704}$ & -27 & $\frac{7168}{112101}$ & 884763 & -884736 & 210 \\
\hline 211 & 12 & 30 & -8 & -2 & $\frac{1}{14348928}$ & $-\frac{993}{434816}$ & 0 & $\frac{256}{112101}$ & 0 & 0 & 211 \\
\hline 212 & 13 & 25 & -3 & -3 & $\frac{1}{14348928}$ & $-\frac{993}{434816}$ & 0 & $\frac{256}{112101}$ & 0 & 0 & 212 \\
\hline 213 & 14 & 20 & 2 & -4 & $\frac{1}{14348928}$ & $-\frac{993}{434816}$ & 0 & $\frac{256}{112101}$ & 0 & 0 & 213 \\
\hline 214 & 15 & 15 & 7 & -5 & $\frac{1}{14348928}$ & $-\frac{993}{434816}$ & 0 & $\frac{256}{112101}$ & 0 & 0 & 214 \\
\hline 215 & 16 & 10 & 12 & -6 & $\frac{1}{14348928}$ & $-\frac{993}{434816}$ & 0 & $\frac{256}{112101}$ & 0 & 0 & 215 \\
\hline 216 & 17 & 5 & 17 & -7 & $\frac{1}{14348928}$ & $-\frac{993}{434816}$ & 0 & $\frac{256}{112101}$ & 0 & 0 & 216 \\
\hline 217 & 18 & 0 & 22 & -8 & $\frac{1}{14348928}$ & $-\frac{993}{434816}$ & 0 & $\frac{256}{112101}$ & 0 & 0 & 217 \\
\hline 218 & 19 & -5 & 27 & -9 & $\frac{1}{14348928}$ & $-\frac{993}{434816}$ & 0 & $\frac{256}{112101}$ & 0 & 0 & 218 \\
\hline 219 & $20-$ & -10 & 32 & -10 & $\frac{61}{3587232}$ & $-\frac{60573}{108704}$ & -243 & $\frac{62464}{112101}$ & 7962867 & -7962624 & 219 \\
\hline
\end{tabular}




\section{Continued}

\begin{tabular}{|c|c|c|c|c|c|c|c|c|c|c|c|}
\hline 220 & 21 & $21-$ & -11 & 1 & $\frac{1}{14348928}$ & $-\frac{993}{434816}$ & 0 & $\frac{256}{112101}$ & 0 & 0 & 220 \\
\hline 221 & 22 & 16 & -6 & 0 & $\frac{1}{14348928}$ & $-\frac{993}{434816}$ & 0 & $\frac{256}{112101}$ & 0 & 0 & 221 \\
\hline 222 & 23 & 11 & -1 & -1 & $\frac{1}{14348928}$ & $-\frac{993}{434816}$ & 0 & $\frac{256}{112101}$ & 0 & 0 & 222 \\
\hline 223 & 24 & 6 & 4 & -2 & $\frac{1}{14348928}$ & $-\frac{993}{434816}$ & 0 & $\frac{256}{112101}$ & 0 & 0 & 223 \\
\hline 224 & 25 & 1 & 9 & -3 & $\frac{1}{14348928}$ & $-\frac{993}{434816}$ & 0 & $\frac{256}{112101}$ & 0 & 0 & 224 \\
\hline 225 & 26 & -4 & 14 & -4 & $\frac{1}{14348928}$ & $-\frac{993}{434816}$ & 0 & $\frac{256}{112101}$ & 0 & 0 & 225 \\
\hline 226 & 27 & -9 & 19 & -5 & $\frac{1}{14348928}$ & $-\frac{993}{434816}$ & 0 & $\frac{256}{112101}$ & 0 & 0 & 226 \\
\hline 227 & $28-$ & -14 & 24 & -6 & $\frac{547}{3587232}$ & $-\frac{543171}{108704}$ & -2187 & $\frac{560128}{112101}$ & 71665803 & -71663616 & 227 \\
\hline 228 & 30 & $12-$ & -14 & 4 & $\frac{1}{14348928}$ & $-\frac{993}{434816}$ & 0 & $\frac{256}{112101}$ & 0 & 0 & 228 \\
\hline 229 & 31 & 7 & -9 & 3 & $\frac{1}{14348928}$ & $-\frac{993}{434816}$ & 0 & $\frac{256}{112101}$ & 0 & 0 & 229 \\
\hline 230 & 32 & 2 & -4 & 2 & $\frac{1}{14348928}$ & $-\frac{993}{434816}$ & 0 & $\frac{256}{112101}$ & 0 & 0 & 230 \\
\hline 231 & 33 & -3 & 1 & 1 & $\frac{1}{14348928}$ & $-\frac{993}{434816}$ & 0 & $\frac{256}{112101}$ & 0 & 0 & 231 \\
\hline 232 & 34 & -8 & 6 & 0 & $\frac{1}{14348928}$ & $-\frac{993}{434816}$ & 0 & $\frac{256}{112101}$ & 0 & 0 & 232 \\
\hline 233 & $35-$ & -13 & 11 & -1 & $\frac{1}{14348928}$ & $-\frac{993}{434816}$ & 0 & $\frac{256}{112101}$ & 0 & 0 & 233 \\
\hline 234 & $36-$ & -18 & 16 & -2 & $\frac{4921}{3587232}$ & $-\frac{4886553}{108704}$ & -19683 & $\frac{5039104}{112101}$ & 644992227 & -644972544 & 234 \\
\hline 235 & 39 & $3-$ & -17 & 7 & $\frac{1}{14348928}$ & $\frac{993}{434816}$ & 0 & $\frac{256}{112101}$ & 0 & 0 & 235 \\
\hline 236 & 40 & $-2-$ & -12 & 6 & $\frac{1}{14348928}$ & $-\frac{993}{434816}$ & 0 & $\frac{256}{112101}$ & 0 & 0 & 236 \\
\hline 237 & 41 & -7 & -7 & 5 & $\frac{1}{14348928}$ & $-\frac{993}{434816}$ & 0 & $\frac{256}{112101}$ & 0 & 0 & 237 \\
\hline 238 & 42 & -12 & -2 & 4 & $\frac{1}{14348928}$ & $-\frac{993}{434816}$ & 0 & $\frac{256}{112101}$ & 0 & 0 & 238 \\
\hline 239 & $43-$ & & 3 & 3 & $\frac{1}{14348928}$ & $-\frac{993}{434816}$ & 0 & $\frac{256}{112101}$ & 0 & 0 & 239 \\
\hline 240 & 44 & & 8 & 2 & $\frac{44287}{3587232}$ & $-\frac{43976991}{108704}$ & -177147 & $\frac{45349888}{112101}$ & 5804930043 & -5804752896 & 240 \\
\hline 241 & $48-$ & $-16-$ & & 10 & $\frac{1}{14348928}$ & $-\frac{993}{434816}$ & 0 & $\frac{256}{112101}$ & 0 & 0 & 241 \\
\hline
\end{tabular}




\section{Continued}

\begin{tabular}{|c|c|c|c|c|c|c|c|c|}
\hline $24249-11-15$ & 9 & $\frac{1}{14348928}$ & $-\frac{993}{434816}$ & 0 & $\frac{256}{112101}$ & 0 & 0 & 242 \\
\hline $24350-16-10$ & 8 & $\frac{1}{14348928}$ & $-\frac{993}{434816}$ & 0 & $\frac{256}{112101}$ & 0 & 0 & 243 \\
\hline $24451-21-5$ & 7 & $\frac{1}{14348928}$ & $\frac{993}{434816}$ & 0 & $\frac{256}{112101}$ & 0 & 0 & 244 \\
\hline $24552-26 \quad 0$ & 6 & $\frac{398581}{3587232}$ & $-\frac{395790933}{108704}$ & -1594323 & $\frac{408146944}{112101}$ & 52244370387 & -52242776064 & 245 \\
\hline $24657-15-23$ & 13 & $\frac{1}{14348928}$ & $-\frac{993}{434816}$ & 0 & $\frac{256}{112101}$ & 0 & 0 & 246 \\
\hline $24758-20-18$ & 12 & $\frac{1}{14348928}$ & $-\frac{993}{434816}$ & 0 & $\frac{256}{112101}$ & 0 & 0 & 247 \\
\hline $24859-25-13$ & 11 & $\frac{1}{14348928}$ & $-\frac{993}{434816}$ & 0 & $\frac{256}{112101}$ & 0 & 0 & 248 \\
\hline $24960-30-8$ & 10 & $\frac{3587227}{3587232}$ & $-\frac{3562116411}{108704}$ & -14348907 & $\frac{3673320448}{112101}$ & 470199333483 & -470184984576 & 249 \\
\hline $25066-24-26$ & 16 & $\frac{1}{14348928}$ & $-\frac{993}{434816}$ & 0 & $\frac{256}{112101}$ & 0 & 0 & 250 \\
\hline $25168-29-21$ & 15 & $\frac{1}{14348928}$ & $-\frac{993}{434816}$ & 0 & $\frac{256}{112101}$ & 0 & 0 & 251 \\
\hline $25268-34-16$ & 14 & $\frac{32285041}{3587232}$ & $-\frac{32059045713}{108704}$ & -129140163 & $\frac{33059881984}{112101}$ & 4231794001347 & -4231664861184 & 252 \\
\hline $25375-33-29$ & 19 & $\frac{1}{14348928}$ & $-\frac{993}{434816}$ & 0 & $\frac{256}{112101}$ & 0 & 0 & 253 \\
\hline $25476-38-24$ & 18 & $\frac{290565367}{3587232}$ & $-\frac{288531409431}{108704}$ & -1162261467 & $\frac{297538935808}{112101}$ & 38086146012123 & -38084983750656 & 254 \\
\hline $25584-42-32$ & 22 & $\frac{60816007}{83424}$ & $\frac{60390294951}{2528}$ & -10460353203 & $\frac{62275591168}{2607}$ & 342775314109107 & -342764853755904 & 255 \\
\hline $256-251171$ & $-25-$ & $\frac{1}{31381105536}$ & $\frac{331}{316980864}$ & $\frac{1}{2187}$ & $-\frac{256}{245164887}$ & $-\frac{3641}{243}$ & $\frac{32768}{2187}$ & 256 \\
\hline $257-17 \quad 7 \quad 63$ & -21 & $-\frac{1}{3486789504}$ & $\frac{331}{35220096}$ & $\frac{1}{243}$ & $-\frac{256}{27240543}$ & $-\frac{3641}{27}$ & $\frac{32768}{243}$ & 257 \\
\hline $\begin{array}{llll}258 & -9 & 3 & 55\end{array}$ & -17 & $\frac{1}{387421056}$ & $\frac{331}{3913344}$ & $\frac{1}{27}$ & $-\frac{256}{3026727}$ & $-\frac{3641}{3}$ & $\frac{32768}{27}$ & 258 \\
\hline $259-1 \quad-1 \quad 47$ & -13 & $-\frac{1}{43046784}$ & $\frac{331}{434816}$ & $\frac{1}{3}$ & $-\frac{256}{336303}$ & -10923 & $\frac{32768}{3}$ & 259 \\
\hline $\begin{array}{llll}260 & 7 & -5 & 39\end{array}$ & -9 & $-\frac{1}{4782976}$ & $\frac{2979}{434816}$ & 3 & $-\frac{256}{37367}$ & -98307 & 98304 & 260 \\
\hline $26115-1931$ & -5 & $-\frac{9}{4782976}$ & $\frac{26811}{434816}$ & 27 & $-\frac{2304}{37367}$ & -884763 & 884736 & 261 \\
\hline $26223-1323$ & -1 & $-\frac{81}{4782976}$ & $\frac{241299}{434816}$ & 243 & $-\frac{20736}{37367}$ & -7962867 & 7962624 & 262 \\
\hline $26331-1715$ & 3 & $-\frac{729}{4782976}$ & $\frac{2171691}{434816}$ & 2187 & $-\frac{186624}{37367}$ & -71665803 & 71663616 & 263 \\
\hline
\end{tabular}


B. Kendirli

\section{Continued}

\begin{tabular}{|c|c|c|c|c|c|c|c|c|c|}
\hline $26439-21$ & 7 & 7 & $-\frac{6561}{4782976}$ & $\frac{19545219}{434816}$ & 19683 & $-\frac{1679616}{37367}$ & -644992227 & 644972544 & 264 \\
\hline $26547-25-$ & & 11 & $-\frac{59049}{4782976}$ & $\frac{175906971}{434816}$ & 177417 & $-\frac{15116544}{37367}$ & -5804930043 & 5804752896 & 265 \\
\hline $26655-29-$ & & 15 & $\frac{531441}{4782976}$ & $\frac{1583162739}{434816}$ & 1594323 & $-\frac{136048896}{37367}$ & -52244370387 & 52242776064 & 266 \\
\hline $26763-33-$ & -17 & 19 & $\frac{4782969}{4782976}$ & $\frac{14248464651}{434816}$ & 14348907 & $-\frac{1224440064}{37367}$ & -470199333483 & 470184984576 & 267 \\
\hline $26871-37-$ & -25 & 23 & $-\frac{43046721}{4782976}$ & $\frac{128236181859}{434816}$ & 129140163 & $-\frac{11019960576}{37367}$ & -4231794001347 & 4231664861184 & 268 \\
\hline $26979-41-$ & -33 & 27 & $-\frac{387420489}{4782976}$ & $\frac{1154125636731}{434816}$ & 1162261467 & $\frac{99179645184}{37367}$ & -38086146012123 & 38084983750656 & 269 \\
\hline $270-228$ & $62-$ & -16 & $\frac{1}{31381105536}$ & $-\frac{331}{316980864}$ & $-\frac{1}{2187}$ & $\frac{256}{245164887}$ & $\frac{3641}{243}$ & $-\frac{32768}{2187}$ & 270 \\
\hline $271-144$ & $54-$ & -12 & $\frac{1}{3486789504}$ & $-\frac{331}{35220096}$ & $-\frac{1}{243}$ & $\frac{256}{27240543}$ & $\frac{3641}{27}$ & $-\frac{32768}{243}$ & 271 \\
\hline $272-6 \quad 0$ & 46 & -8 & $\frac{1}{387421056}$ & $-\frac{331}{3913344}$ & $-\frac{1}{27}$ & $\frac{256}{3026727}$ & $\frac{3641}{3}$ & $-\frac{32768}{27}$ & 272 \\
\hline $\begin{array}{llll}273 & 2 & -4 & 3\end{array}$ & 38 & -4 & $\frac{1}{43046784}$ & $-\frac{331}{434816}$ & $-\frac{1}{3}$ & $\frac{256}{336303}$ & 10923 & $-\frac{32768}{3}$ & 273 \\
\hline $274 \quad 10-8$ & 30 & 0 & $\frac{1}{4782976}$ & $-\frac{2979}{434816}$ & -3 & $\frac{256}{37367}$ & 98307 & -98304 & 274 \\
\hline $27518-12$ & 22 & 4 & $\frac{9}{4782976}$ & $\frac{26811}{434816}$ & -27 & $\frac{2304}{37367}$ & 884763 & -884736 & 275 \\
\hline $27626-16$ & 14 & 8 & $\frac{81}{4782976}$ & $-\frac{241299}{434816}$ & -243 & $\frac{20736}{37367}$ & 7962867 & -7962624 & 276 \\
\hline $27734-20$ & 6 & 12 & $\frac{729}{4782976}$ & $-\frac{2171691}{434816}$ & -2187 & $\frac{186624}{37367}$ & 71665803 & -71663616 & 277 \\
\hline $27842-24-$ & -21 & 16 & $\frac{6561}{4782976}$ & $-\frac{19545219}{434816}$ & -19683 & $\frac{1679616}{37367}$ & 644992227 & -644972544 & 278 \\
\hline $27950-28-$ & -102 & 20 & $\frac{59049}{4782976}$ & $-\frac{175906971}{434816}$ & -177147 & $\frac{15116544}{37367}$ & 5804930043 & -5804752896 & 279 \\
\hline $28058-32-$ & -182 & 24 & $\frac{531441}{4782976}$ & $-\frac{1583162739}{434816}$ & -1594323 & $\frac{136048896}{37367}$ & 52244370387 & -52242776064 & 280 \\
\hline $28166-36-$ & -262 & 28 & $\frac{4782969}{4782976}$ & $-\frac{14248464651}{434816}$ & -14348907 & $\frac{1224440064}{37367}$ & 470199333483 & -470184984576 & 281 \\
\hline $28274-40-$ & -34 & 32 & $\frac{43046721}{4782976}$ & $-\frac{128236181859}{434816}$ & -129140163 & $\frac{11019960576}{37367}$ & 4231794001347 & -4231664861184 & 282 \\
\hline $283-1955$ & 53 & -7 & $-\frac{1}{31381105536}$ & $\frac{331}{316980864}$ & $\frac{1}{2187}$ & $-\frac{256}{245164887}$ & $-\frac{3641}{243}$ & $\frac{32768}{2187}$ & 283 \\
\hline $284-11 \quad 1 \quad 4$ & 45 & -3 & $-\frac{1}{3486789504}$ & $\frac{331}{35220096}$ & $\frac{1}{243}$ & $-\frac{256}{27240543}$ & $-\frac{3641}{27}$ & $\frac{32768}{243}$ & 284 \\
\hline $285-3-3$ & 37 & 1 & $-\frac{1}{387421056}$ & $\frac{331}{3913344}$ & $\frac{1}{27}$ & $-\frac{256}{3026727}$ & $-\frac{3641}{3}$ & $\frac{32768}{27}$ & 285 \\
\hline
\end{tabular}




\section{Continued}

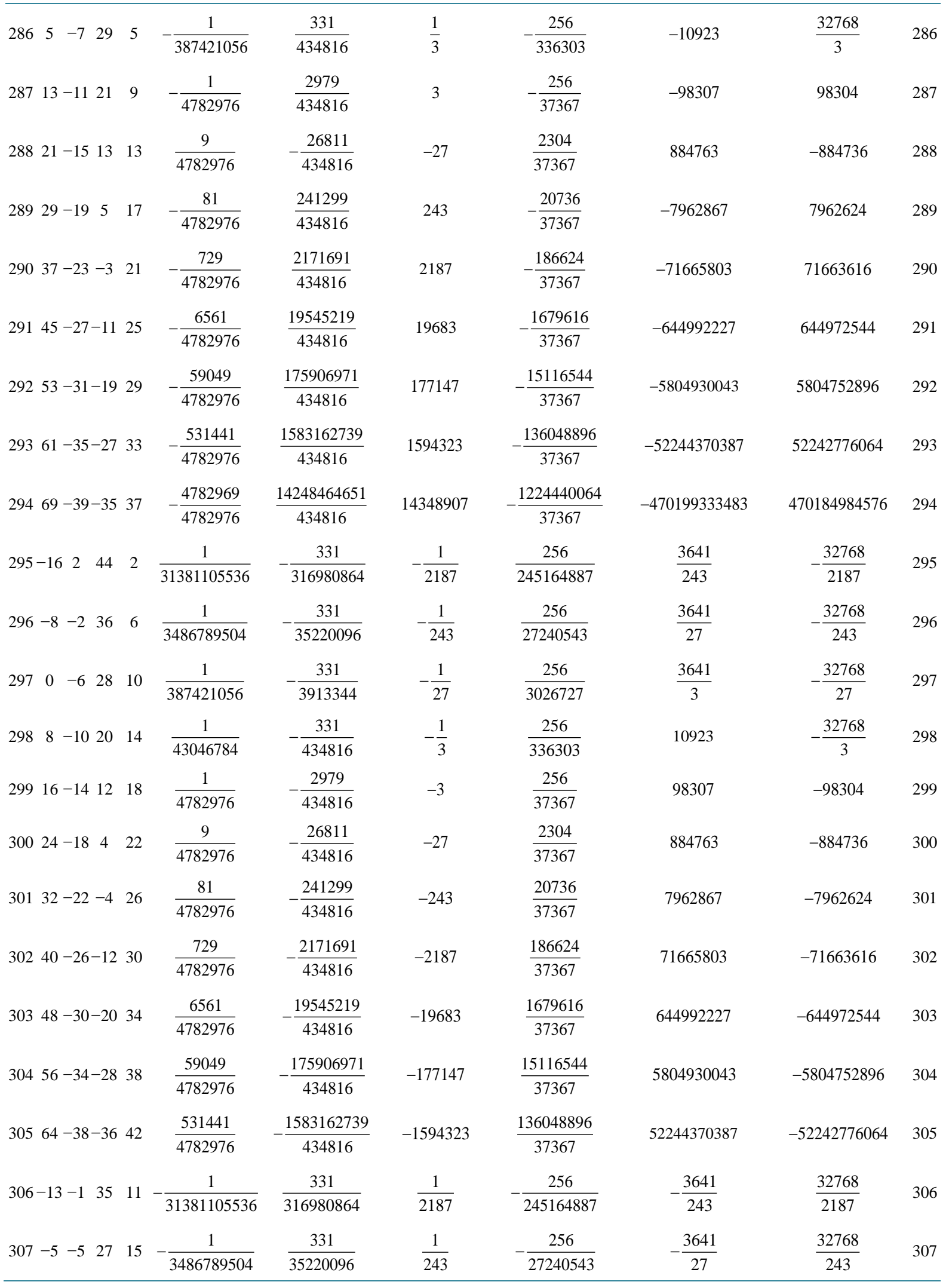




\section{Continued}

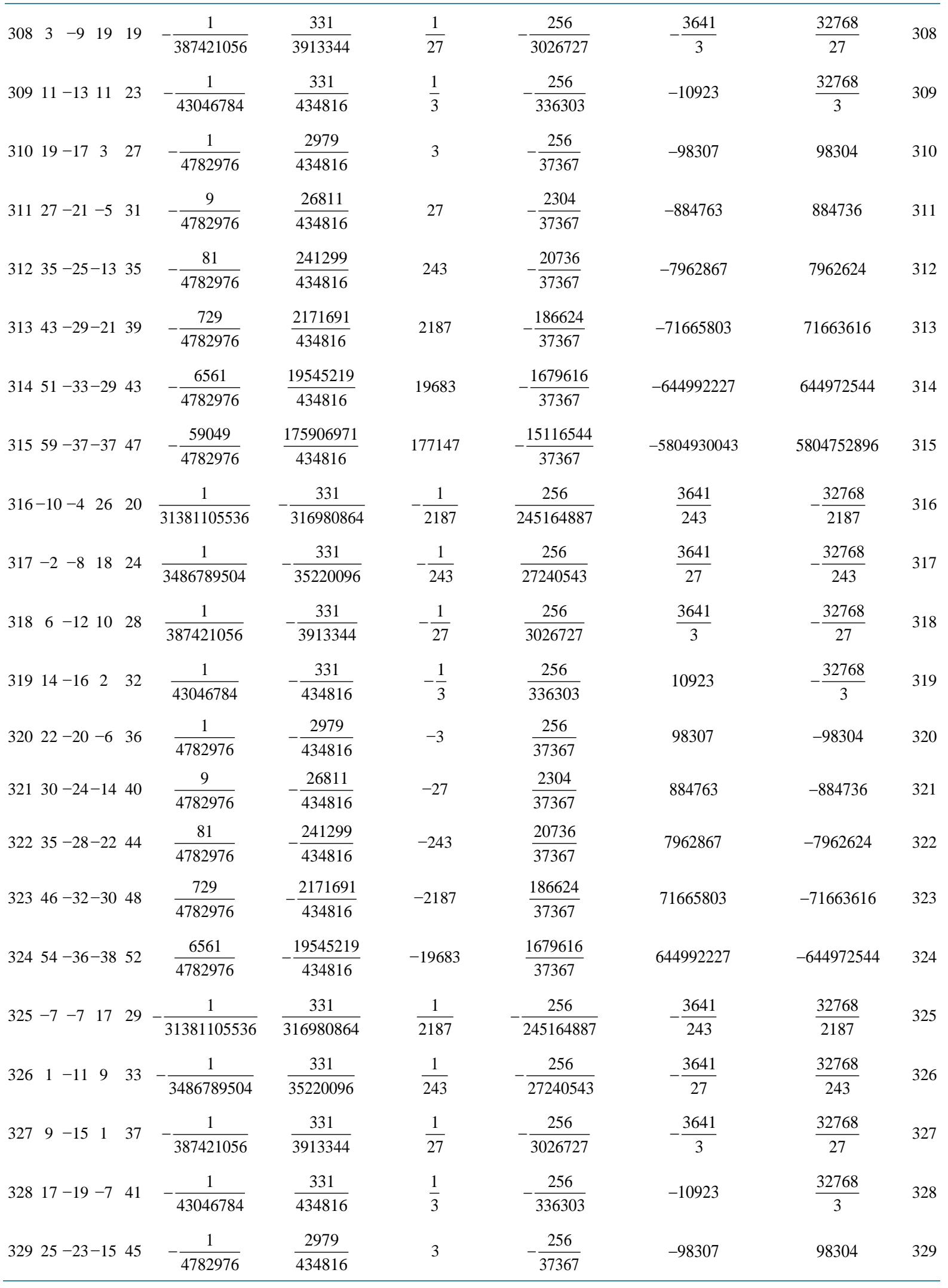




\section{Continued}

\begin{tabular}{|c|c|c|c|c|c|c|c|}
\hline $33033-27-2349$ & $-\frac{9}{4782976}$ & $\frac{26811}{434816}$ & 27 & $-\frac{2304}{37367}$ & -884763 & 884736 & 330 \\
\hline $33141-31-3153$ & $-\frac{81}{4782976}$ & $\frac{241299}{434816}$ & 243 & $-\frac{20736}{37367}$ & -7962867 & 7962624 & 331 \\
\hline $33249-35-3957$ & $\frac{729}{4782976}$ & $\frac{2171691}{434816}$ & 2187 & $-\frac{186624}{37367}$ & -71665803 & 71663616 & 332 \\
\hline $\begin{array}{llll}333 & -4 & -10 & 8\end{array} 38$ & $\frac{1}{31381105536}$ & $-\frac{331}{316980864}$ & $-\frac{1}{2187}$ & $\frac{256}{245164887}$ & $\frac{3641}{243}$ & $-\frac{32768}{2187}$ & 333 \\
\hline $\begin{array}{lllll}334 & 4 & -14 & 0 & 42\end{array}$ & $\frac{1}{3486789504}$ & $-\frac{331}{35220096}$ & $-\frac{1}{243}$ & $\frac{256}{27240543}$ & $\frac{3641}{27}$ & $-\frac{32768}{243}$ & 334 \\
\hline $335 \quad 12-18-8 \quad 46$ & $\frac{1}{387421056}$ & $-\frac{331}{3913344}$ & $-\frac{1}{27}$ & $\frac{256}{3026727}$ & $\frac{3641}{3}$ & $-\frac{32768}{27}$ & 335 \\
\hline $33620-22-1650$ & $\frac{1}{43046784}$ & $-\frac{331}{434816}$ & $-\frac{1}{3}$ & $\frac{256}{336303}$ & 10923 & $-\frac{32768}{3}$ & 336 \\
\hline $33728-26-2454$ & $\frac{1}{4782976}$ & $-\frac{2979}{434816}$ & -3 & $\frac{256}{37367}$ & 98307 & -98304 & 337 \\
\hline $33836-30-3258$ & $\frac{9}{4782976}$ & $-\frac{26811}{434816}$ & -27 & $\frac{2304}{37367}$ & 884763 & -884736 & 338 \\
\hline $33944-34-4062$ & $\frac{81}{4782976}$ & $\frac{241299}{434816}$ & -243 & $\frac{20736}{37367}$ & 7962867 & -7962624 & 339 \\
\hline $340-1-13-1 \quad 47$ & $\frac{1}{31381105536}$ & $\frac{331}{316980864}$ & $\frac{1}{2187}$ & $\frac{256}{245164887}$ & $-\frac{3641}{243}$ & $\frac{32768}{2187}$ & 340 \\
\hline $\begin{array}{lllll}341 & 7 & -17 & -9 & 51\end{array}$ & $-\frac{1}{3486789504}$ & $\frac{331}{35220096}$ & $\frac{1}{243}$ & $-\frac{256}{27240543}$ & $-\frac{3641}{27}$ & $\frac{32768}{243}$ & 341 \\
\hline $34215-21-1755$ & $\frac{1}{387421056}$ & $\frac{331}{3913344}$ & $\frac{1}{27}$ & $-\frac{256}{3026727}$ & $-\frac{3641}{3}$ & $\frac{32768}{27}$ & 342 \\
\hline $34323-25-2559$ & $\frac{1}{43046784}$ & $\frac{331}{434816}$ & $\frac{1}{3}$ & $-\frac{256}{336303}$ & -10923 & $\frac{32768}{3}$ & 343 \\
\hline $34431-29-3363$ & $-\frac{1}{4782976}$ & $\frac{2979}{434816}$ & 3 & $-\frac{256}{37367}$ & -98307 & 98304 & 344 \\
\hline $34539-33-4167$ & $\frac{9}{4782976}$ & $\frac{26811}{434816}$ & 27 & $-\frac{2304}{37367}$ & -884763 & 884736 & 345 \\
\hline $346 \quad 2-16-1056$ & $\frac{1}{31381105536}$ & $-\frac{331}{316980864}$ & $\frac{1}{2187}$ & $\frac{256}{245164887}$ & $\frac{3641}{243}$ & $-\frac{32768}{2187}$ & 346 \\
\hline $347 \quad 10-20-1860$ & $\frac{1}{3486789504}$ & $\frac{331}{35220096}$ & $-\frac{1}{243}$ & $\frac{256}{27240543}$ & $\frac{3641}{27}$ & $-\frac{32768}{243}$ & 347 \\
\hline
\end{tabular}




\section{Continued}

\begin{tabular}{|c|c|c|c|c|c|c|c|}
\hline \multirow{2}{*}{$34818-24-2664$} & 1 & 331 & 1 & 256 & 3641 & 32768 & \multirow{2}{*}{348} \\
\hline & $\overline{387421056}$ & 3913344 & $\overline{27}$ & $\overline{3026727}$ & 3 & 27 & \\
\hline \multirow{2}{*}{$26-28-3468$} & 1 & 331 & 1 & 256 & \multirow{2}{*}{10923} & 32768 & \multirow{2}{*}{349} \\
\hline & $\overline{43046784}$ & $\overline{434816}$ & $\overline{3}$ & $\overline{336303}$ & & 3 & \\
\hline \multirow{2}{*}{$350 \quad 34-32-4272$} & 1 & 2979 & \multirow{2}{*}{-3} & 256 & \multirow{2}{*}{98307} & \multirow{2}{*}{-98304} & \multirow{2}{*}{350} \\
\hline & 4782976 & $\overline{434816}$ & & $\overline{37367}$ & & & \\
\hline \multirow{2}{*}{$351 \quad 5-19-1965$} & 1 & 331 & 1 & 256 & 3641 & 32768 & \multirow{2}{*}{351} \\
\hline & $\overline{31381105536}$ & $\overline{316980864}$ & $\overline{2187}$ & 245164887 & $\overline{243}$ & $\overline{2187}$ & \\
\hline \multirow{2}{*}{$352 \quad 13-23-2769$} & 1 & 331 & 1 & 256 & 3641 & 32768 & \multirow{2}{*}{352} \\
\hline & $\overline{3486789504}$ & $\overline{35220096}$ & $\overline{243}$ & $\overline{27240543}$ & 27 & 243 & \\
\hline \multirow{2}{*}{$35321-27-3573$} & 1 & 331 & 1 & 256 & 3641 & 32768 & \multirow{2}{*}{353} \\
\hline & $\overline{387421056}$ & $\overline{3913344}$ & $\overline{27}$ & 3026727 & 3 & 27 & \\
\hline \multirow{2}{*}{$35429-31-4377$} & 1 & 331 & 1 & 256 & \multirow{2}{*}{-10923} & 32768 & \multirow{2}{*}{354} \\
\hline & $\overline{43046784}$ & $\overline{434816}$ & $\overline{3}$ & $\overline{336303}$ & & 3 & \\
\hline \multirow{2}{*}{$355 \quad 8 \quad-22-28 \quad 74$} & 1 & 331 & 1 & 256 & 3641 & 32768 & \multirow{2}{*}{355} \\
\hline & $\overline{31381105536}$ & 316980864 & $\overline{2187}$ & $\overline{245164887}$ & $\overline{243}$ & 2187 & \\
\hline \multirow{2}{*}{$356 \quad 16-26-3678$} & 1 & 331 & 1 & 256 & 3641 & 32768 & \multirow{2}{*}{356} \\
\hline & $\overline{3486789504}$ & $\overline{35220096}$ & $\overline{243}$ & $\overline{27240543}$ & 27 & 243 & \\
\hline \multirow{2}{*}{$35724-30-4482$} & 1 & 331 & 1 & 256 & 3641 & 32768 & \multirow{2}{*}{357} \\
\hline & $\overline{387421056}$ & 3913344 & $\overline{27}$ & $\overline{3026727}$ & 3 & 27 & \\
\hline \multirow{2}{*}{$35811-25-3783$} & 1 & 331 & 1 & 256 & 3641 & 32768 & \multirow{2}{*}{358} \\
\hline & 31381105536 & $\overline{316980864}$ & $\overline{2187}$ & 245164887 & 243 & 2187 & \\
\hline \multirow{2}{*}{$359 \quad 19-29-4587$} & 1 & 331 & 1 & 256 & 3641 & 32768 & \multirow{2}{*}{359} \\
\hline & 3486789504 & $\overline{35220096}$ & $\overline{243}$ & $\overline{27240543}$ & 27 & 243 & \\
\hline \multirow{2}{*}{$360 \quad 14-28-4692$} & 1 & 331 & 1 & 256 & 3641 & 32768 & \multirow{2}{*}{360} \\
\hline & $\overline{31381105536}$ & $\overline{316980864}$ & $\overline{2187}$ & $\overline{245164887}$ & $\overline{243}$ & $\overline{2187}$ & \\
\hline
\end{tabular}

Remark $4 S_{16}\left(\Gamma_{0}(12)\right)$ is 27 dimensional, $M_{16}\left(\Gamma_{0}(12)\right)$ is 33 dimensional, see [18] (Chapter 3, p. 87 and Chapter 5, p. 197), and generated by

$$
\begin{aligned}
& \Delta_{1,16}, \Delta_{1,16}(2 z), \Delta_{1,16}(3 z), \Delta_{1,16}(4 z), \Delta_{1,16}(6 z), \Delta_{1,16}(12 z), \\
& \Delta_{2,16}, \Delta_{2,16}(2 z), \Delta_{2,16}(3 z), \Delta_{2,16}(6 z), \\
& \Delta_{3,16,1}, \Delta_{3,16,1}(2 z), \Delta_{3,16,1}(4 z), \Delta_{3,16,2}(z), \Delta_{3,16,2}(2 z), \Delta_{3,16,2}(4 z), \\
& \Delta_{4,16}, \Delta_{4,16}(3 z), \\
& \Delta_{6,16,1}, \Delta_{6,16,1}(2 z), \Delta_{6,16,2}, \Delta_{6,16,2}(2 z), \Delta_{6,16,3}, \Delta_{6,16,3}(2 z), \\
& \Delta_{12,16,1}, \Delta_{12,16,2}, \Delta_{12,16,3}\left(\text { conjugate of } \Delta_{12,16,2}\right)
\end{aligned}
$$

where $\Delta_{1,16}$ is the unique newform in $S_{16}\left(\Gamma_{0}(1)\right) ; \Delta_{2,16}$ is the unique newform in $S_{16}\left(\Gamma_{0}(2)\right) ; \Delta_{3,16,1}, \Delta_{3,16,2}$ are the unique newforms in $S_{16}\left(\Gamma_{0}(3)\right), \Delta_{4,16}$ is the unique newform in $S_{16}\left(\Gamma_{0}(4)\right), \Delta_{6,14,1}, \Delta_{6,14,2}, \Delta_{6,14,3}$ are the unique newforms in $S_{14}\left(\Gamma_{0}(6)\right)$ and $\Delta_{12,16,1}, \Delta_{12,16,2}, \Delta_{12,16,3}$ are the unique newforms in $S_{16}\left(\Gamma_{0}(12)\right)$. By 
Table 3. Expression of $\mathrm{f}_{i}$ in terms of newforms.

$$
\begin{aligned}
& f_{1}=\frac{29}{2211840000} \Delta_{1,16}(z)-\frac{29}{10240000} \Delta_{1,16}(2 z)+\frac{17997}{81920000} \Delta_{1,16}(3 z)+\frac{29}{67500} \Delta_{1,16}(4 z) \\
& -\frac{485919}{10240000} \Delta_{1,16}(6 z)+\frac{17997}{2500} \Delta_{1,16}(12 z)-\frac{97}{4992000000} \Delta_{2,16}(z)-\frac{97}{39000000} \Delta_{2,16}(2 z) \\
& +\frac{297861}{1664000000} \Delta_{2,16}(3 z)+\frac{297861}{13000000} \Delta_{2,16}(6 z)-\frac{517}{23601217536} \Delta_{3,16,1}(z)-\frac{517}{327794688} \Delta_{3,1,1,1}(2 z) \\
& -\frac{517}{720252} \Delta_{3,16,1}(4 z)+\frac{1861}{96111360000} \Delta_{3,16,2}(z)+\frac{24193}{5339520000} \Delta_{3,16,2}(2 z)+\frac{29776}{46929375} \Delta_{3,16,2}(4 z) \\
& -\frac{11}{6523846656} \Delta_{4,16}(z)-\frac{598035}{2174615552} \Delta_{4,16}(3 z)-\frac{77}{2723217408} \Delta_{6,16,1}(z)+\frac{77}{21275136} \Delta_{6,16,1}(2 z) \\
& +\frac{1367}{59050819584} \Delta_{6,1,6,2}(z)-\frac{1367}{461334528} \Delta_{6,16,2}(2 z)+\frac{1547}{110592000000} \Delta_{6,16,3}(z)+\frac{1547}{864000000} \Delta_{6,1,3,3}(2 z) \\
& -\frac{3133}{50594512896} \Delta_{12,1,1,1}(z)+(10111 t+98584027470) \Delta_{12,16,2}(z)+(10111 t+99288359730) \Delta_{12,16,3}(z)
\end{aligned}
$$

$$
\begin{aligned}
& f_{2}=\frac{6341}{2972712960000} \Delta_{1,16}(z)-\frac{6341}{13762560000} \Delta_{1,16}(2 z)+\frac{1148571}{36700160000} \Delta_{1,16}(3 z) \\
& +\frac{6341}{90720000} \Delta_{1,16}(4 z)-\frac{31011417}{4587520000} \Delta_{1,16}(6 z)+\frac{1148571}{1120000} \Delta_{1,16}(12 z) \\
& -\frac{4643}{1916928000000} \Delta_{2,16}(z)-\frac{4643}{14976000000} \Delta_{2,16}(2 z)+\frac{5019003}{212992000000} \Delta_{2,16}(3 z) \\
& +\frac{5019003}{1664000000} \Delta_{2,16}(6 z)-\frac{4253}{1510477922304} \Delta_{3,16,1}(z)-\frac{4253}{20978860032} \Delta_{3,16,1}(2 z) \\
& -\frac{4253}{46096128} \Delta_{3,16,1}(4 z)+\frac{59381}{24604508160000} \Delta_{3,16,2}(z)+\frac{771953}{1366917120000} \Delta_{3,16,2}(2 z) \\
& +\frac{59381}{750870000} \Delta_{3,16,2}(4 z)-\frac{797}{1252578557952} \Delta_{4,16}(z)-\frac{5327451}{139175395328} \Delta_{4,16}(3 z) \\
& -\frac{157}{33888927744} \Delta_{6,16,1}(z)+\frac{157}{264757248} \Delta_{6,16,1}(2 z)+\frac{191}{52489617408} \Delta_{6,16,2}(z) \\
& -\frac{191}{410075136} \Delta_{6,16,2}(2 z)+\frac{497}{294912000000} \Delta_{6,16,3}(z)+\frac{497}{2304000000} \Delta_{6,16,3}(2 z) \\
& -\frac{1}{117116928} \Delta_{12,16,1}(z)+\frac{5599 t+33630313230}{7373558809991577600} \Delta_{12,16,2}(z) \\
& +\frac{-5599 t+34020339570}{7373558809991577600} \Delta_{12,16,3}(z) \\
& f_{3}=\frac{899}{495452160000} \Delta_{1,16}(z)-\frac{899}{2293760000} \Delta_{1,16}(2 z)+\frac{512007}{18350080000} \Delta_{1,16}(3 z) \\
& +\frac{899}{15120000} \Delta_{1,16}(4 z)-\frac{13824189}{2293760000} \Delta_{1,16}(6 z)+\frac{512007}{560000} \Delta_{1,16}(12 z) \\
& -\frac{677}{319488000000} \Delta_{2,16}(z)-\frac{677}{2496000000} \Delta_{2,16}(2 z)+\frac{2228151}{106496000000} \Delta_{2,16}(3 z) \\
& +\frac{2228151}{832000000} \Delta_{2,16}(6 z)-\frac{217}{83915440128} \Delta_{3,16,1}(z)-\frac{217}{1165492224} \Delta_{3,16,1}(2 z) \\
& -\frac{217}{2560896} \Delta_{3,16,1}(4 z)+\frac{2953}{1366917120000} \Delta_{3,16,2}(z)+\frac{38389}{75939840000} \Delta_{3,16,2}(2 z) \\
& +\frac{2953}{41715000} \Delta_{3,16,2}(4 z)-\frac{107}{208763092992} \Delta_{4,16}(z)-\frac{2396487}{69587697664} \Delta_{4,16}(3 z) \\
& -\frac{611}{152500174848} \Delta_{6,16,1}(z)+\frac{611}{1191407616} \Delta_{6,16,1}(2 z)+\frac{95}{29525409792} \Delta_{6,16,2}(z) \\
& -\frac{95}{230667264} \Delta_{6,16,2}(2 z)+\frac{7}{4608000000} \Delta_{6,16,3}(z)+\frac{7}{36000000} \Delta_{6,16,3}(2 z) \\
& -\frac{1535}{202378051584} \Delta_{12,16,1}(z)+\frac{10949 t+80222205690}{199086087869772559520} \Delta_{12,16,2}(z) \\
& +\frac{-10949 t+80984913030}{199086087869772559520} \Delta_{12,16,3}(z) \text {, }
\end{aligned}
$$




\section{Continued}

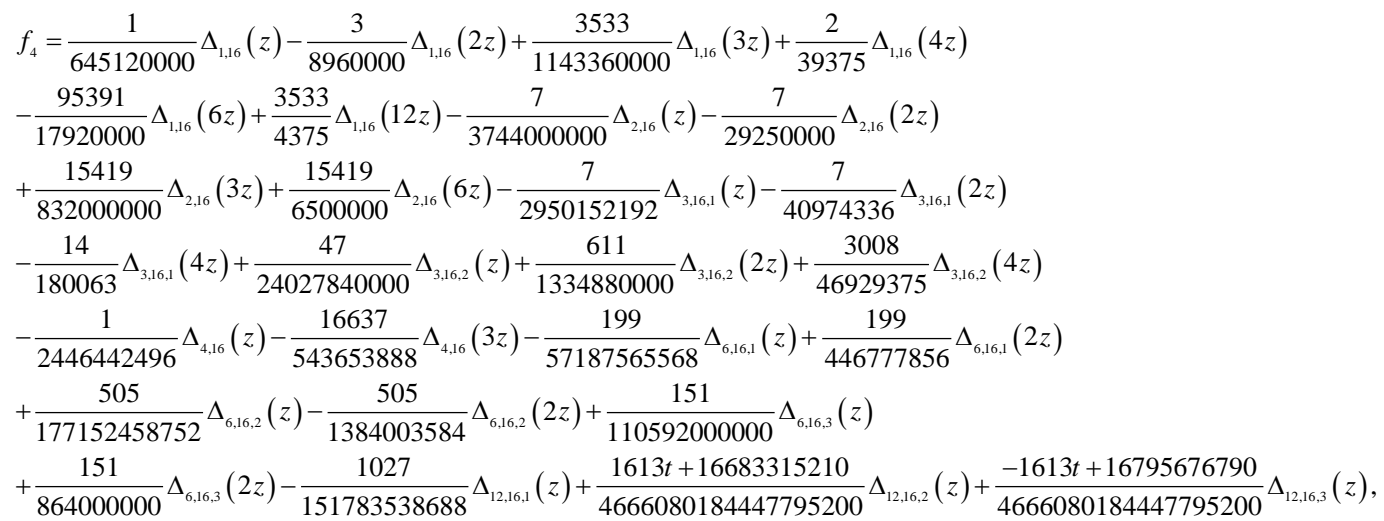

$$
\begin{aligned}
& f_{5}=\frac{187}{139345920000} \Delta_{1,16}(z)-\frac{187}{645120000} \Delta_{1,16}(2 z)+\frac{112591}{5160960000} \Delta_{1,16}(3 z) \\
& +\frac{187}{4252500} \Delta_{1,16}(4 z)-\frac{337773}{71680000} \Delta_{1,16}(6 z)+\frac{112591}{157500} \Delta_{1,16}(12 z) \\
& -\frac{151}{89856000000} \Delta_{2,16}(z)-\frac{151}{702000000} \Delta_{2,16}(2 z)+\frac{496013}{29952000000} \Delta_{2,16}(3 z) \\
& +\frac{496013}{234000000} \Delta_{2,16}(6 z)-\frac{151}{70803652608} \Delta_{3,16,1}(z)-\frac{151}{983384064} \Delta_{3,16,1}(2 z) \\
& -\frac{151}{2160756} \Delta_{3,16,1}(4 z)+\frac{523}{288334080000} \Delta_{3,16,2}(z)+\frac{6799}{16018560000} \Delta_{3,16,2}(2 z) \\
& +\frac{8368}{140788125} \Delta_{3,16,2}(4 z)-\frac{19}{58714619904} \Delta_{4,16}(z)-\frac{532223}{19571539968} \Delta_{4,16}(3 z) \\
& -\frac{29}{9531260928} \Delta_{6,16,1}(z)+\frac{29}{74462976} \Delta_{6,16,1}(2 z)+\frac{37}{14762704896} \Delta_{6,16,2}(z) \\
& -\frac{37}{11533632} \Delta_{6,16,2}(2 z)+\frac{11}{9216000000} \Delta_{6,16,3}(z)+\frac{11}{72000000} \Delta_{6,16,3}(2 z) \\
& -\frac{229}{37945884672} \Delta_{12,16,1}(z)+\frac{811 t+29641066470}{9332160368895590400} \Delta_{12,16,2}(z) \\
& +\frac{-811 t+29697560730}{9332160368895590400} \Delta_{12,16,3}(z),
\end{aligned}
$$$$
f_{6}=-\frac{527}{46448640000} \Delta_{1,16}(z)+\frac{527}{215040000} \Delta_{1,16}(2 z)-\frac{61137}{573440000} \Delta_{1,16}(3 z)
$$$$
-\frac{527}{1417500} \Delta_{1,16}(4 z)+\frac{1650699}{1680000} \Delta_{1,16}(6 z)-\frac{61137}{17500} \Delta_{1,16}(12 z)
$$$$
-\frac{629}{29952000000} \Delta_{2,16}(z)-\frac{629}{234000000} \Delta_{2,16}(2 z)+\frac{587709}{3328000000} \Delta_{2,16}(3 z)
$$$$
+\frac{587709}{26000000} \Delta_{2,16}(6 z)-\frac{5}{23601217536} \Delta_{3,16,1}(z)-\frac{5}{327794688} \Delta_{3,16,1}(2 z)
$$$$
-\frac{5}{720252} \Delta_{3,16,1}(4 z)+\frac{1217}{96111360000} \Delta_{3,16,2}(z)+\frac{15821}{5339520000} \Delta_{3,16,2}(2 z)
$$$$
+\frac{19472}{46929375} \Delta_{3,16,2}(4 z)+\frac{15}{2174615552} \Delta_{4,16}(z)+\frac{3105}{2174615552} \Delta_{4,16}(3 z)
$$$$
+\frac{23}{1059028992} \Delta_{6,16,1}(z)-\frac{23}{8273664} \Delta_{6,16,1}(2 z)-\frac{25}{1640300544} \Delta_{6,16,2}(z)
$$$$
+\frac{25}{12814848} \Delta_{6,16,2}(2 z)+\frac{371}{27648000000} \Delta_{6,16,3}(z)+\frac{371}{216000000} \Delta_{6,16,3}(2 z)
$$$$
-\frac{5}{1405403136} \Delta_{12,16,1}(z)+\frac{2249 t-655556670}{345635569218355200} \Delta_{12,16,2}(z)
$$$$
-\frac{2249 t+498891330}{345635569218355200} \Delta_{12,16,3}(z) \text {, }
$$ 


\section{Continued}

$f_{7}=-\frac{121859542812417387532763153}{47140015663246252474728429649920000} \Delta_{1,16}(z)$

951140139433279244737601494210315446579282556996701576273333095511

$-\frac{9144107841139294607117668839427510483552119903913404486424985600000}{61,16}(2 z)$ 21302225876428712615716637

$-\frac{21302225876428712615716637}{482700167554923278701690880000} \Delta_{1,16}(3 z)$

379409075464544302185315894706225042187514699339478015118057635393799

$\frac{3538502808642107946656172137145584421995225454703187117164134400000}{1,16}(4 z)$

_ 26407113627491667178436267902753339023532093370734285799292395083112121

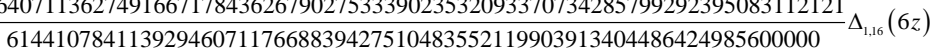

$+\frac{1969424271653554293105803759254452282916421075920629041644919622330879781}{5376094360968827812279602344907167310810491592422892562186240000}$ 5376094360996882781227960234499071673108104915924228925621862400000 36720058380953696452825273

$+\frac{36720058380953696452825273}{8473327360904972429388029952000000} \Delta_{2,16}(z)$

3319662368135994360962746630782643327556238540571350141802886489844913

$+\frac{3317971515435332436725819181355325468164389950718946708122793082880000000}{17,16}(2 z)$ 145634444765492327012883569

$-\frac{145634444765492327012883569}{3663349485907899883003904000000} \Delta_{2,16}(3 z)$

6360303256651524200423491734929785744997662518794838633048666208256499673

1996835048370270747313242372813940907154438968771856458088120320000000 118647854757936555056855

$+\frac{118647854757936555056855}{25979419509406884495097382043648} \Delta_{3,16,1}(z)$

83242702806757313708276633133965682061060259117149650553416928792793

$+\frac{83242702806757313708276026}{196680264924278187527365120752681923591083620668152773695847499038720} \Delta_{3,16,1}(2 z)$

4975663774361885770024976058051545503547488196197091586599181725785319

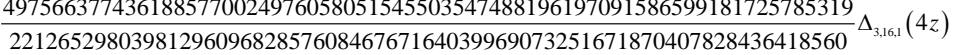
23062692139224271815795569

$-\frac{23062692139224271815795569}{5501398456967329570302282792960000} \Delta_{3,16,2}(z)$

7613847806508625869819880062082885878544900885490999765032138052460227

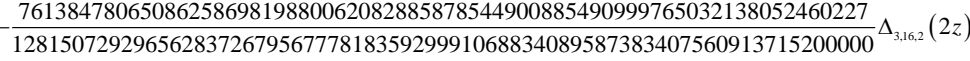

$-\frac{7281724628231679140155914143013882991757662371586746783126233676507081919}{57667828183453276770580550018261684959809753403143225334024111718400000} \Delta_{3,1,2,2}(4 z)$

$+\frac{1692717589838997548293}{5958892194165451739291342340096} \Delta_{4,16}(z)+\frac{189860527089807200143294831}{3310495663425250966272967966720} \Delta_{4,16}(3 z)$ 5696552799715101190393631

$+\frac{5696552799715101190393631}{1022939643182896076994459417968640} \Delta_{6,16,1}(z)$

7634874489069267246353244951577999282250372020067519553124845005509999

$+\frac{7634874489069267246353244951577999282250372020067519553124845005509999}{8578285400928133256001232574366973128934185609141740206580425534996480} \Delta_{6,16,1}(2 z)$ 181480184405315924801251

$\frac{181480184405315924801251}{39610068890164772906176436109312} \Delta_{6,16,2}(z)$

88332540284712745105418185210418212158613084627116337754328435011033

$-\frac{883254028466336690874198543968105187305368504179602104977923978883497984}{33216669033,2} \Delta_{6}(2 z)$ 38342834754288358036199

$\frac{38342834754288358036199}{12363804514939162105138176000000} \Delta_{6,16,3}(z)$

62612746747939787550994711742495477820597898524627498312231359186779

$-\frac{261276274774031995618888327081674049262427529223173375885527040000000}{1296022}$ 3515277290858932535620781

$+\frac{3515277290858932535620781}{271502025596663394871461785370624} \Delta_{12,16,1}(z)$ $+\frac{-419382167695689085876267 t-3747578352221313324333197997030}{568267368514024907209190826420480245760} \Delta_{12,16,2}(z)$ $+\frac{419382167695689085876267 t-3776792514022995026055338756250}{568267368514024907209190826420480245760} \Delta_{12,16,3}(z)$

$+\frac{207767842132293}{12804139123145291671075245260800} E_{16}(\mathrm{z})$

$+\frac{389352657132658554776560538059030483705486122469087773}{7219369180216201846903536789484910023526820456630348097126400} E_{16}(2 z)$ 2343816

$\frac{2343816}{190054142030133966098225} E_{16}(3 z)$

12728309157919516667880818187040166611739623824482340625581

$-\frac{127283015387360432403693807073578969820047053640913260696194252800}{1443873836} E_{16}(4 z)$

63628032768920311754117531280298428267819697026418673529

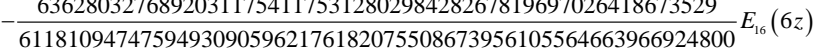

6935936546517597174149866024128733438060610717383151483691

$+\frac{6935936546517597174149866024128733438060610717383151483691}{3609684590108100923451768394742455011763410228315174048563200} E_{16}(12 z)$ 


\section{Continued}

$$
\begin{aligned}
& f_{s}=\frac{112591}{1720320000} \Delta_{1,16}(z)-\frac{1013319}{71680000} \Delta_{1,16}(2 z)+\frac{99379467}{573440000} \Delta_{1,16}(3 z)+\frac{112591}{52500} \Delta_{1,16}(4 z)-\frac{2683245609}{71680000} \Delta_{1,16}(6 z)+\frac{99379467}{17500} \Delta_{1,16}(12 z) \\
& +\frac{496013}{9984000000} \Delta_{2,16}(z)+\frac{496013}{78000000} \Delta_{2,16}(2 z)-\frac{722228319}{3328000000} \Delta_{2,16}(3 z)-\frac{722228319}{26000000} \Delta_{2,16}(6 z)+\frac{1359}{32374784} \Delta_{3,16,1}(z)+\frac{12231}{4046848} \Delta_{3,16,1}(2 z) \\
& +\frac{1359}{988} \Delta_{3,1,1,1}(4 z)+\frac{4707}{131840000} \Delta_{3,16,2}(z)+\frac{550719}{65920000} \Delta_{3,16,2}(2 z)+\frac{75312}{64375} \Delta_{3,16,2}(4 z)-\frac{532223}{6523846656} \Delta_{4,16}(z)-\frac{90876411}{2174615552} \Delta_{4,16}(3 z) \\
& +\frac{261}{4358144} \Delta_{6,16,1}(z)-\frac{261}{34048} \Delta_{6,16,1}(2 z)+\frac{333}{6750208} \Delta_{6,16,2}(z)-\frac{333}{52736} \Delta_{6,16,2}(2 z)+\frac{24057}{1024000000} \Delta_{6,16,3}(z)+\frac{24057}{8000000} \Delta_{6,16,3}(2 z) \\
& -\frac{687}{5783552} \Delta_{12,16,1}(z)+\frac{-811 t-29641066470}{474122865868800} \Delta_{12,16,2}(z)+\frac{811 t-29697560730}{474122865868800} \Delta_{12,16,3}(z),
\end{aligned}
$$

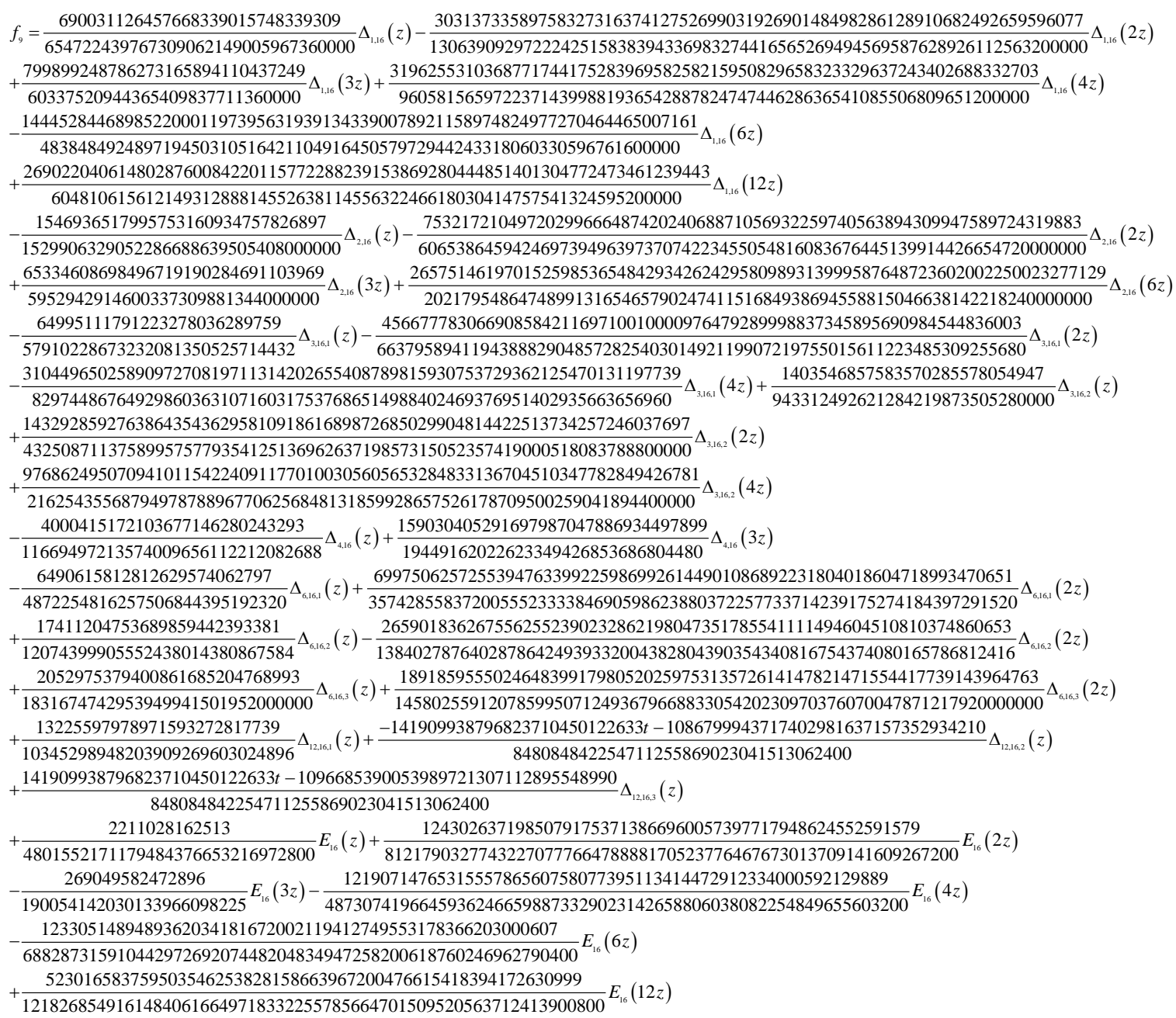

$$
\begin{aligned}
& f_{10}=\frac{53}{725760000} \Delta_{1,16}(z)-\frac{53}{3360000} \Delta_{1,16}(2 z)+\frac{243}{8960000} \Delta_{1,16}(3 z)+\frac{848}{354375} \Delta_{1,16}(4 z)-\frac{6561}{1120000} \Delta_{1,16}(6 z) \\
& +\frac{3888}{4375} \Delta_{1,16}(12 z)+\frac{659}{29952000000} \Delta_{2,16}(z)+\frac{659}{234000000} \Delta_{2,16}(2 z)+\frac{17661}{3328000000} \Delta_{2,16}(3 z)+\frac{176661}{26000000} \Delta_{2,16}(6 z) \\
& -\frac{1}{40974336} \Delta_{3,16,1}(z)-\frac{1}{569088} \Delta_{3,16,1}(2 z)-\frac{16}{20007} \Delta_{3,1,1,1}(4 z)-\frac{7}{166860000} \Delta_{3,1,6,2}(z)-\frac{91}{9270000} \Delta_{3,16,2}(2 z) \\
& -\frac{7168}{5214375} \Delta_{3,16,2}(4 z)-\frac{27}{271826944} \Delta_{4,16}(z)-\frac{5589}{271826944} \Delta_{4,16}(3 z)-\frac{1}{58834944} \Delta_{6,16,1}(z)+\frac{1}{459648} \Delta_{6,1,1,1}(2 z) \\
& -\frac{1}{45563904} \Delta_{6,16,2}(z)+\frac{1}{355968} \Delta_{6,16,2}(2 z)+\frac{71}{6912000000} \Delta_{6,16,3}(z)+\frac{71}{54000000} \Delta_{6,16,3}(2 z)+\frac{1}{19519488} \Delta_{12,1,1,1}(z) \\
& +\frac{-7 t+1783074}{64006586892288} \Delta_{12,16,2}(z)+\frac{7 t+1295454}{64006586892288} \Delta_{12,1,, 3}(z)
\end{aligned}
$$




\section{Continued}

$$
\begin{aligned}
& f_{11}=\frac{836863}{6720000} \Delta_{1,16}(z)-\frac{7531767}{280000} \Delta_{1,16}(2 z)+\frac{686090331}{2240000} \Delta_{1,16}(3 z) \\
& +\frac{53559232}{13125} \Delta_{1,16}(4 z)-\frac{18524438937}{280000} \Delta_{1,16}(6 z)+\frac{43909781184}{4375} \Delta_{1,16}(12 z) \\
& +\frac{12840549}{104000000} \Delta_{2,16}(z)+\frac{12840549}{812500} \Delta_{2,16}(2 z)-\frac{60595434261}{104000000} \Delta_{2,16}(3 z) \\
& -\frac{60595434261}{812500} \Delta_{2,16}(6 z)+\frac{11079}{126464} \Delta_{3,16,1}(z)+\frac{99711}{15808} \Delta_{3,16,1}(2 z) \\
& +\frac{709056}{247} \Delta_{3,16,1}(4 z)+\frac{43101}{515000} \Delta_{3,16,2}(z)+\frac{5042817}{257500} \Delta_{3,16,2}(2 z) \\
& +\frac{176541696}{64375} \Delta_{3,16,2}(4 z)+\frac{1062583}{12741888} \Delta_{4,16}(z)+\frac{52612659}{4247296} \Delta_{4,16}(3 z) \\
& +\frac{6855}{68096} \Delta_{6,16,1}(z)-\frac{6855}{532} \Delta_{6,16,1}(2 z)+\frac{17601}{210944} \Delta_{6,16,2}(z) \\
& -\frac{17601}{1648} \Delta_{6,16,2}(2 z)+\frac{2024433}{32000000} \Delta_{6,16,3}(z)+\frac{2024433}{250000} \Delta_{6,16,3}(2 z) \\
& +\frac{1401}{11296} \Delta_{12,16,1}(z)+\frac{7193 t+58049091810}{926021222400} \Delta_{12,16,2}(z) \\
& +\frac{-7193 t+58550156190}{926021222400} \Delta_{12,16,3}(z) \text {, } \\
& f_{12}=-\frac{473}{120960000} \Delta_{1,16}(z)+\frac{473}{560000} \Delta_{1,16}(2 z)-\frac{102789}{4480000} \Delta_{1,16}(3 z) \\
& -\frac{15136}{118125} \Delta_{1,16}(4 z)+\frac{2775303}{560000} \Delta_{1,16}(6 z)-\frac{3289248}{4375} \Delta_{1,16}(12 z) \\
& +\frac{1457}{624000000} \Delta_{2,16}(z)+\frac{1457}{4875000} \Delta_{2,16}(2 z)+\frac{452709}{208000000} \Delta_{2,16}(3 z) \\
& +\frac{452709}{1625000} \Delta_{2,16}(6 z)-\frac{11}{6829056} \Delta_{3,16,1}(z)-\frac{11}{94848} \Delta_{3,16,1}(2 z) \\
& -\frac{352}{6669} \Delta_{3,16,1}(4 z)+\frac{37}{27810000} \Delta_{3,16,2}(z)+\frac{481}{1545000} \Delta_{3,16,2}(2 z) \\
& +\frac{75776}{1738125} \Delta_{3,16,2}(4 z)+\frac{1}{1225728} \Delta_{6,16,1}(z)-\frac{1}{9576} \Delta_{6,16,1}(2 z) \\
& +\frac{5}{1898496} \Delta_{6,16,2}(z)-\frac{5}{14832} \Delta_{6,16,2}(2 z)-\frac{51}{32000000} \Delta_{6,16,3}(z)-\frac{51}{250000} \Delta_{6,16,3}(2 z), \\
& f_{13}=-\frac{43}{17418240000} \Delta_{1,16}(z)+\frac{43}{80640000} \Delta_{1,16}(2 z)+\frac{1601}{645120000} \Delta_{1,16}(3 z) \\
& -\frac{86}{1063125} \Delta_{1,16}(4 z)-\frac{4803}{8960000} \Delta_{1,16}(6 z)+\frac{3202}{39375} \Delta_{1,16}(12 z) \\
& -\frac{113}{89856000000} \Delta_{2,16}(z)-\frac{113}{702000000} \Delta_{2,16}(2 z)-\frac{83381}{29952000000} \Delta_{2,16}(3 z) \\
& -\frac{83381}{234000000} \Delta_{2,16}(6 z)+\frac{7}{8850456576} \Delta_{3,16,1}(z)+\frac{7}{122923008} \Delta_{3,16,1}(2 z) \\
& +\frac{14}{540189} \Delta_{3,16,1}(4 z)+\frac{53}{36041760000} \Delta_{3,16,2}(z)+\frac{689}{2002320000} \Delta_{3,16,2}(2 z) \\
& +\frac{6784}{140788125} \Delta_{3,16,2}(4 z)-\frac{1}{611610624} \Delta_{4,16}(z)-\frac{23}{67956736} \Delta_{4,16}(3 z) \\
& +\frac{5}{4765630464} \Delta_{6,16,1}(z)-\frac{5}{37231488} \Delta_{6,16,1}(2 z)+\frac{1}{3690676224} \Delta_{6,16,2}(z) \\
& -\frac{1}{28833408} \Delta_{6,16,2}(2 z)+\frac{1}{6912000000} \Delta_{6,16,3}(z)+\frac{1}{54000000} \Delta_{6,16,3}(2 z) \\
& +\frac{1}{1185808896} \Delta_{12,16,1}(z)+\frac{-619 t+137004570}{291630011527987200} \Delta_{12,16,2}(z) \\
& +\frac{619 t+93885030}{291630011527987200} \Delta_{12,16,3}(z) \text {, }
\end{aligned}
$$




\section{Continued}

$$
\begin{aligned}
& f_{14}=-\frac{5999}{737280000} \Delta_{1,16}(z)+\frac{17997}{10240000} \Delta_{1,16}(2 z)-\frac{1712421}{81920000} \Delta_{1,16}(3 z) \\
& -\frac{5999}{22500} \Delta_{1,16}(4 z)+\frac{46235367}{10240000} \Delta_{1,16}(6 z)-\frac{1712421}{2500} \Delta_{1,16}(12 z) \\
& -\frac{99287}{14976000000} \Delta_{2,16}(z)-\frac{99287}{117000000} \Delta_{2,16}(2 z)+\frac{51549777}{1664000000} \Delta_{2,16}(3 z) \\
& +\frac{51549777}{13000000} \Delta_{2,16}(6 z)-\frac{517}{97124352} \Delta_{3,16,1}(z)-\frac{1551}{4046848} \Delta_{3,16,1}(2 z) \\
& -\frac{517}{2964} \Delta_{3,16,1}(4 z)-\frac{1861}{395520000} \Delta_{3,16,2}(z)-\frac{7259}{65920000} \Delta_{3,16,2}(2 z) \\
& -\frac{29776}{193125} \Delta_{3,16,2}(4 z)+\frac{199345}{19571539968} \Delta_{4,16}(z)+\frac{5845851}{2174615552} \Delta_{4,16}(3 z) \\
& -\frac{77}{11206656} \Delta_{6,16,1}(z)+\frac{77}{87552} \Delta_{6,16,1}(2 z)-\frac{1367}{243007488} \Delta_{6,16,2}(z) \\
& +\frac{1367}{1898496} \Delta_{6,16,2}(2 z)-\frac{13923}{4096000000} \Delta_{6,16,3}(z)-\frac{13923}{32000000} \Delta_{6,16,3}(2 z) \\
& +\frac{3133}{208207872} \Delta_{12,16,1}(z)+\frac{10111 t+98584027470}{12801317378457600} \Delta_{12,16,2}(z) \\
& +\frac{-10111 t+99288359730}{12801317378457600} \Delta_{12,16,3}(z) \text {, } \\
& f_{15}=-\frac{419}{362880000} \Delta_{1,16}(2 z)+\frac{21017}{45360000} \Delta_{1,16}(4 z)-\frac{82989}{4480000} \Delta_{1,16}(6 z)+\frac{4081527}{560000} \Delta_{1,16}(12 z) \\
& -\frac{6229}{1872000000} \Delta_{2,16}(2 z)+\frac{6495309}{208000000} \Delta_{2,16}(6 z)-\frac{49}{1843845121} \Delta_{3,16,1}(2 z) \\
& -\frac{19313}{23048064} \Delta_{3,16,1}(4 z)+\frac{1249}{750870000} \Delta_{3,16,2}(2 z)+\frac{295697}{375435000} \Delta_{3,16,2}(4 z) \\
& +\frac{115}{33094656} \Delta_{6,16,1}(2 z)-\frac{143}{51259392} \Delta_{6,16,2}(2 z)+\frac{691}{288000000} \Delta_{6,16,3}(2 z) \\
& f_{16}=-\frac{181}{181440000} \Delta_{1,16}(2 z)+\frac{1151}{2835000} \Delta_{1,16}(4 z)-\frac{36411}{2240000} \Delta_{1,16}(6 z)+\frac{227481}{35000} \Delta_{1,16}(12 z) \\
& -\frac{2771}{936000000} \Delta_{2,16}(2 z)+\frac{2873691}{104000000} \Delta_{2,16}(6 z)-\frac{23}{92192256} \Delta_{3,16,1}(2 z) \\
& -\frac{1067}{1440504} \Delta_{3,16,1}(4 z)+\frac{551}{375435000} \Delta_{3,16,2}(2 z)+\frac{32632}{46929375} \Delta_{3,16,2}(4 z) \\
& +\frac{461}{148925952} \Delta_{6,16,1}(2 z)-\frac{577}{230667264} \Delta_{6,16,2}(2 z)+\frac{103}{48000000} \Delta_{6,16,3}(2 z) \\
& f_{17}=\frac{3016467424350077822543980849801255783}{58457986859248401255323017927213440000} \Delta_{1,16}(2 z)+\frac{108228459021795622087139221463494625081}{7307248357406050156915377240901680000} \Delta_{1,16}(4 z) \\
& +\frac{3101772145184949025052089352942949167419}{2165110624416607453900852515822720000} \Delta_{1,16}(6 z)-\frac{33047294429459075615400456620997496510267}{270638828052075931737606564477840000} \Delta_{1,16}(12 z) \\
& -\frac{129972863878150771751944331208617492329}{2110982858806192267553331202927152000000} \Delta_{2,16}(2 z)+\frac{747069455447250702545628283720848817140227}{703660952935397422517777067642384000000} \Delta_{2,16}(6 z) \\
& -\frac{29332800951290575846259872152295739797}{207923367660974713584932910163512763392} \Delta_{3,16,1}(2 z)+\frac{194818674082894111350832567271094277033}{25990420957621839198116613770439095424} \Delta_{3,16,1}(4 z) \\
& +\frac{167683605463202612943147532114209875299}{846727403414426061932569337789482170000} \Delta_{3,16,2}(2 z)+\frac{17818757149853870998254485587531747229597}{423363701707213030966284668894741085000} \Delta_{3,16,2}(4 z) \\
& -\frac{14234744479773888084180581806762011023}{47982315614071087750369133114656791552} \Delta_{6,16,1}(2 z)+\frac{46113624946307175246214068266935663717}{520229316657823372451370601137857845248} \Delta_{6,16,2}(2 z) \\
& +\frac{17432668584289644612623510125778279197}{108255531220830372695042625791136000000} \Delta_{6,16,3}(2 z)-\frac{1723086865299786724260468}{95851251601776892490402324919235} E_{16}(2 z) \\
& +\frac{28164675296010249628106929524}{95851251601776892490402324919235} E_{16}(4 z)+\frac{6645452032544826975883600572}{19170250320355378498080464983847} E_{16}(6 z) \\
& -\frac{61390212371869084720800671916}{95851251601776892490402324919235} E_{16}(12 z) \text {, }
\end{aligned}
$$




\section{Continued}

$$
\begin{aligned}
& f_{18}=-\frac{53}{60480000} \Delta_{1,16}(2 z)+\frac{169}{472500} \Delta_{1,16}(4 z)-\frac{95987}{6720000} \Delta_{1,16}(6 z)+\frac{304751}{52500} \Delta_{1,16}(12 z)-\frac{823}{312000000} \Delta_{2,16}(2 z) \\
& +\frac{7629047}{312000000} \Delta_{2,16}(6 z)-\frac{7}{30730752} \Delta_{3,16,1}(2 z)-\frac{157}{240084} \Delta_{3,16,1}(4 z)+\frac{163}{125145000} \Delta_{3,16,2}(2 z)+\frac{9616}{15643125} \Delta_{3,16,2}(4 z) \\
& +\frac{137}{49641984} \Delta_{6,16,1}(2 z)-\frac{43}{19222272} \Delta_{6,16,2}(2 z)-\frac{23}{12000000} \Delta_{6,16,3}(2 z)
\end{aligned}
$$

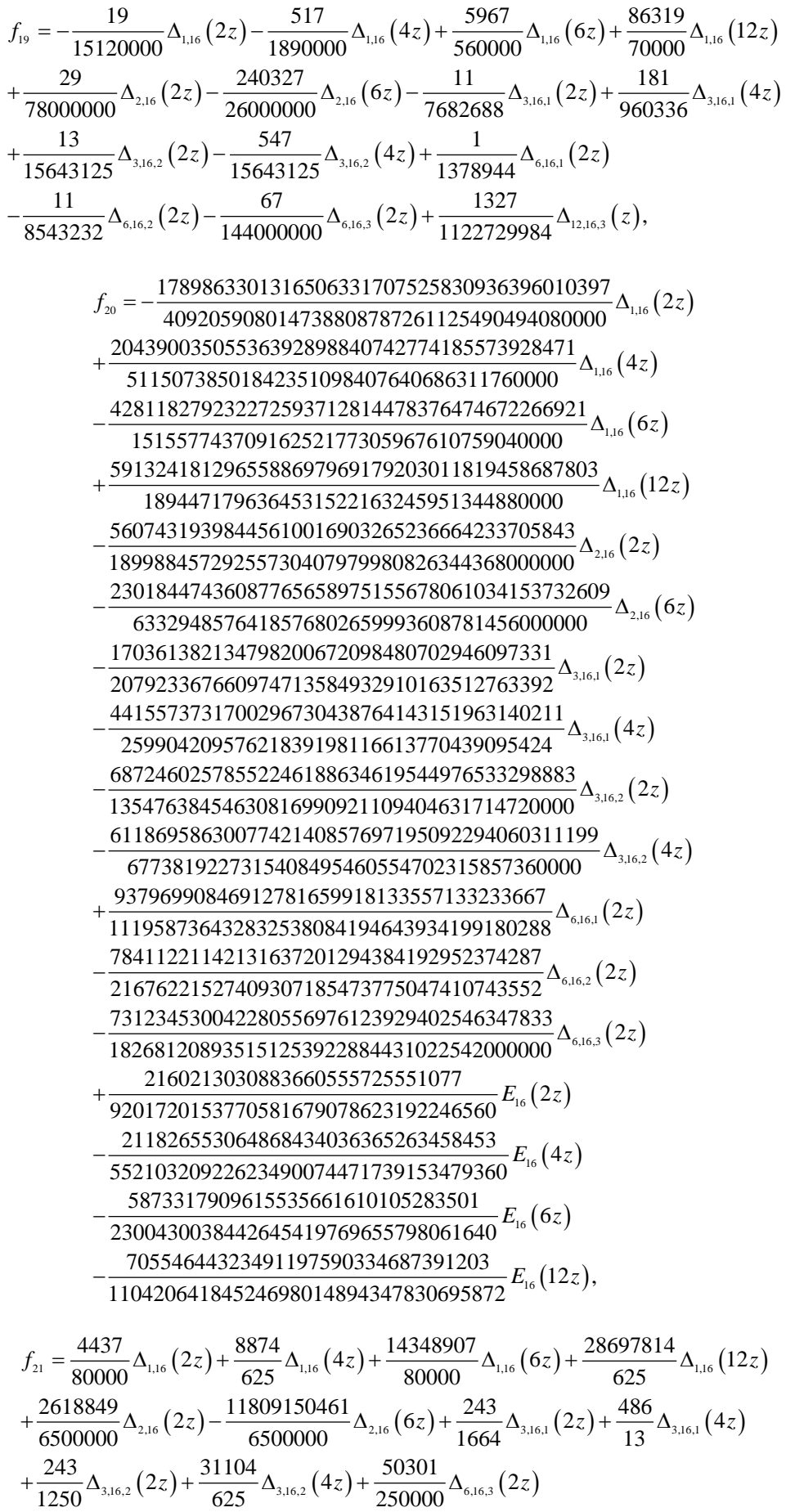




\section{Continued}

$$
\begin{aligned}
& f_{22}=\frac{29}{2211840000} \Delta_{1,16}(z)-\frac{29}{10240000} \Delta_{1,16}(2 z)+\frac{17997}{81920000} \Delta_{1,16}(3 z) \\
& +\frac{29}{67500} \Delta_{1,16}(4 z)-\frac{485919}{10240000} \Delta_{1,16}(6 z)+\frac{17997}{2500} \Delta_{1,16}(12 z) \\
& -\frac{97}{4992000000} \Delta_{2,16}(z)-\frac{97}{39000000} \Delta_{2,16}(2 z)+\frac{297861}{1664000000} \Delta_{2,16}(3 z) \\
& +\frac{297861}{13000000} \Delta_{2,16}(6 z)-\frac{517}{23601217536} \Delta_{3,16,1}(z)-\frac{517}{327794688} \Delta_{3,16,1}(2 z) \\
& -\frac{517}{720252} \Delta_{3,16,1}(4 z)+\frac{1861}{96111360000} \Delta_{3,16,2}(z)+\frac{24193}{5339520000} \Delta_{3,16,2}(2 z) \\
& +\frac{29776}{46929375} \Delta_{3,16,2}(4 z)-\frac{11}{6523846656} \Delta_{4,16}(z)-\frac{598035}{2174615552} \Delta_{4,16}(3 z) \\
& -\frac{77}{2723217408} \Delta_{6,16,1}(z)+\frac{77}{21275136} \Delta_{6,16,1}(2 z)+\frac{1367}{59050819584} \Delta_{6,16,2}(z) \\
& -\frac{1367}{461334528} \Delta_{6,16,2}(2 z)+\frac{1547}{110592000000} \Delta_{6,16,3}(z) \\
& +\frac{1547}{864000000} \Delta_{6,16,3}(2 z)-\frac{3133}{50594512896} \Delta_{12,16,1}(z) \\
& +\frac{10111 t+9858027470}{3110720122965196800} \Delta_{12,16,2}(z) \\
& +\frac{-10111 t+99288359730}{3110720122965196800} \Delta_{12,16,3}(z), \\
& f_{23}=\frac{29}{2211840000} \Delta_{1,16}(z)-\frac{29}{10240000} \Delta_{1,16}(2 z)+\frac{17997}{81920000} \Delta_{1,16}(3 z) \\
& +\frac{29}{67500} \Delta_{1,16}(4 z)-\frac{485919}{10240000} \Delta_{1,16}(6 z)+\frac{17997}{2500} \Delta_{1,16}(12 z) \\
& -\frac{97}{4992000000} \Delta_{2,16}(z)-\frac{97}{39000000} \Delta_{2,16}(2 z)+\frac{297861}{1664000000} \Delta_{2,16}(3 z) \\
& +\frac{297861}{13000000} \Delta_{2,16}(6 z)-\frac{517}{23601217536} \Delta_{3,16,1}(z)-\frac{517}{327794688} \Delta_{3,16,1}(2 z) \\
& -\frac{517}{720252} \Delta_{3,16,1}(4 z)+\frac{1861}{96111360000} \Delta_{3,16,2}(z)+\frac{24193}{5339520000} \Delta_{3,16,2}(2 z) \\
& +\frac{29776}{46929375} \Delta_{3,16,2}(4 z)-\frac{11}{6523846656} \Delta_{4,16}(z)-\frac{598035}{2174615552} \Delta_{4,16}(3 z) \\
& -\frac{77}{2723217408} \Delta_{6,16,1}(z)+\frac{77}{21275136} \Delta_{6,16,1}(2 z)+\frac{1367}{59050819584} \Delta_{6,16,2}(z) \\
& -\frac{1367}{461334528} \Delta_{6,16,2}(2 z)+\frac{1547}{110592000000} \Delta_{6,16,3}(z) \\
& +\frac{1547}{864000000} \Delta_{6,16,3}(2 z)-\frac{3133}{50594512896} \Delta_{12,16,1}(z) \\
& +\frac{10111 t+98584027470}{3110720122965196800} \Delta_{12,16,2}(z) \\
& +\frac{-10111 t+99288359730}{3110720122965196800} \Delta_{12,16,3}(z) \text {, } \\
& f_{24}=\frac{47}{120960000} \Delta_{1,16}(2 z)-\frac{1021}{15120000} \Delta_{1,16}(4 z)+\frac{12771}{4480000} \Delta_{1,16}(6 z) \\
& -\frac{36153}{560000} \Delta_{1,16}(12 z)-\frac{23}{624000000} \Delta_{2,16}(2 z)-\frac{354051}{208000000} \Delta_{2,16}(6 z) \\
& -\frac{11}{61461504} \Delta_{3,16,1}(2 z)+\frac{181}{7682688} \Delta_{3,16,1}(4 z)-\frac{37}{250290000} \Delta_{3,16,2}(2 z) \\
& -\frac{5461}{125145000} \Delta_{3,16,2}(4 z)+\frac{1}{11031552} \Delta_{6,16,1}(2 z)-\frac{5}{17086464} \Delta_{6,16,2}(2 z) \\
& +\frac{17}{96000000} \Delta_{6,16,3}(2 z)
\end{aligned}
$$




\section{Continued}

$f_{25}=-\frac{878685640055594915565020963806423215499}{3682853172132649279085350129414446720000} \Delta_{1,16}(2 z)-\frac{17148102875315060668515774406980627337693}{460356646516581159885668766176805840000} \Delta_{1,16}(4 z)$

$-\frac{764441387353080174716126299059936723266257}{136401969338246269595753708496831360000} \Delta_{1,16}(6 z)+\frac{8855772537293604258134340281862162119463101}{17050246167280783699469213562103920000} \Delta_{1,16}(12 z)$

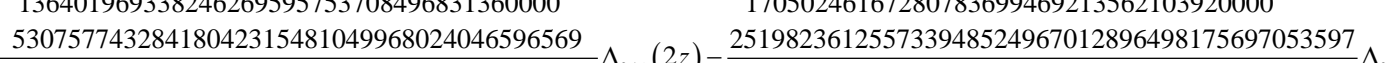

$+\frac{53075774328418042315481049968024046596569}{5170989611563301573671819827437099312000000} \Delta_{2,16}(2 z)-\frac{251982361255733948524967012896498175697053597}{56996537187767191223939942479033104000000} \Delta_{2,16}(6 z)$

$+\frac{259187560276944972438562034995924792999}{467827577237193105566099047867903717632} \Delta_{3,16,1}(2 z)-\frac{1769292743442904696991373356477046214087}{116956894309298276391524761966975929408} \Delta_{3,16,1}(4 z)$

$-\frac{710764737548767893239234786758666502816663}{975429968733418823346319877133483459840000} \Delta_{3,16,2}(2 z)-\frac{72586214200056483796638296836978093625927939}{487714984366709411673159938566741729920000} \Delta_{3,16,2}(4 z)$

$+\frac{30108033285015100885446651141776646637}{27989684108208134521048660983549795072} \Delta_{6,16,1}(2 z)-\frac{135958201607280717584912238482447969503}{346819544438548914967580400758571896832} \Delta_{6,16,2}(2 z)$

$-\frac{30559265484757601996764253053290796906477}{52612188173323561129790716134492096000000} \Delta_{6,16,3}(2 z)+\frac{90291524865603541349728867831}{1325047702142963761787321739683504640} E_{16}(2 z)$

$\frac{4482405405080632376282908663946101}{3975143106428891285361965219050513920} E_{16}(4 z)+\frac{4357238443934985195238757084499917}{1325047702142963761787321739683504640} E_{16}(6 z)$

8589580801298920020057411776157143

$\frac{8589580801298920020057411776157143}{3975143106428891285361965219050513920} E_{16}(12 z)$,

$f_{26}=-\frac{17792737526473618862640692662183084879}{9820941792353731410894267011771857920000} \Delta_{1,16}(2 z)-\frac{604268046694798092302561343487389700001}{409205908014738808787261125490494080000} \Delta_{1,16}(4 z)$

$-\frac{29167555593395337307004937806014706713147}{363738584901990052255343222658216960000} \Delta_{1,16}(6 z)+\frac{82564496730812223539805579771600831471307}{15155774370916252177305967610759040000} \Delta_{1,16}(12 z)$

$+\frac{593550430198936540934438713918027951799}{455972297502137529791519539832264832000000} \Delta_{2,16}(2 z)-\frac{7800901820729198328618141073459831300126837}{151990765834045843263839846610754944000000} \Delta_{2,16}(6 z)$

$+\frac{2041414681166865480787119341583304897}{262640043361231217159915254943384543232} \Delta_{3,16,1}(2 z)-\frac{9394248114814513474717929333256082789}{10943335140051300714996468955974355968} \Delta_{3,1,1,1}(4 z)$

$-\frac{8144673349886004718952772233084099485337}{650286645822279215564213251422322306560000} \Delta_{3,16,2}(2 z)-\frac{307946999365475981365841493188766727499087}{108381107637046535927368875237053717760000} \Delta_{3,16,2}(4 z)$

$+\frac{8188454816410230827335594606759308077}{424264685429681196950632334908544262144} \Delta_{6,16,1}(2 z)-\frac{22045961439581124404486299502237056595}{6242751799893880469416447213654294142976} \Delta_{6,16,2}(2 z)$

$368525190674577063898391405322386940923 \quad 905023961291585293054669339$

$-\frac{368525190674577063898391405322386940923}{35074792115549040753193810756328064000000} \Delta_{6,16,3}(2 z)+\frac{905023961291585293054669339}{883365134761975841191547826455669760} E_{16}(2 z)$

$\frac{2921161715984479748627613509695}{76673026952395168238309565291133952} E_{16}(4 z)-\frac{64837155038755209796398929244811}{883365134761975841191547826455669760} E_{16}(6 z)$

79442058594716316954243942123947

$+\frac{79442058594716316954243942123947}{883365134761975841191547826455669760} E_{16}(12 z)$,

$f_{27}=-\frac{1718117326894260275110190763342510895373}{409205908014738808787261125490494080000} \Delta_{1,16}(2 z)+\frac{18748429703159920733684137268006789090839}{51150738501842351098407640686311760000} \Delta_{1,16}(4 z)$

$-\frac{419804987919864607826025399977026475308889}{15155774370916252177305967610759040000} \Delta_{1,16}(6 z)+\frac{5726686569858080308795744013462033853133627}{1894471796364531522163245951344880000} \Delta_{1,16}(12 z)$

$-\frac{52187892132874314105286378229620058902387}{18998845729255730407979980826344368000000} \Delta_{2,16}(2 z)+\frac{17326978349901625674142837699510091394810881}{6332948576418576802659993608781456000000} \Delta_{2,16}(6 z)$

$-\frac{140885403710693675549133231988596900547}{207923367660974713584932910163512763392} \Delta_{3,16,1}(2 z)-\frac{4375167571183184235491084873160673210483}{25990420957621839198116613770439095424} \Delta_{3,16,1}(4 z)$

$-\frac{66902063768040144707537539347361592601347}{13547638454630816990921109404631714720000} \Delta_{3,16,2}(2 z)-\frac{5991982143626077356701775004399608840684191}{6773819227315408495460554702315857360000} \Delta_{3,16,2}(4 z)$

$+\frac{109650796883140948080361736018993518595}{111958736432832538084194643934199180288} \Delta_{6,16,1}(2 z)-\frac{76078747466447625991206995777656558031}{21676221527409307185473775047410743552} \Delta_{6,16,2}(2 z)$

$-\frac{713951426227343767144987679152365694297}{182681208935151253922884431022542000000} \Delta_{6,16,3}(2 z)+\frac{2160213030883660555725551077}{9201720153770581679078623192246560} E_{16}(2 z)$

$\frac{211826553064868434036365263458453}{55210320922623490074471739153479360} E_{16}(4 z)-\frac{5873317909615535661610105283501}{2300430038442645419769655798061640} E_{16}(6 z)$

$+\frac{70554644323491197590334687391203}{11042064184524698014894347830695872} E_{16}(12 z)$. 
taking $t$ as a root of $x^{2}-69660 x-65283075900$, we see $f_{1}, \cdots, f_{27}$ as linear combinations in Table 3.

\section{References}

[1] Williams, K.S. (2012) Fourier Series of a Class of Eta Quotients. International Journal of Number Theory, 8, 993-1004. http://dx.doi.org/10.1142/S1793042112500595

[2] Yao, O.X.M., Xia, E.X.W. and Jin, J. (2013) Explicit Formulas for the Fourier Coefficients of a Class of Eta Quotients. International Journal of Number Theory, 9, 487-503. http://dx.doi.org/10.1142/S179304211250145X

[3] Köhler, G. (2011) Eta Products and Theta Series Identities. Springer-Verlag, Berlin. http://dx.doi.org/10.1007/978-3-642-16152-0

[4] Gordon, B. (1961) Some Identities in Combinatorial Analysis. Quarterly Journal of Mathematics, 12, 285-290.

[5] Kac, V.G. (1978) Infinite-Dimensional Algebras, Dedekind’s $\eta$-Function, Classical Möbius Function and the Very Strange Formula. Advances in Mathematics, 30, 85-136. http://dx.doi.org/10.1016/0001-8708(78)90033-6

[6] Macdonald, I.G. (1972) Affine Root Systems and Dedekind’s $\eta$-Function. Inventiones Mathematicae, 15, 91-143. http://dx.doi.org/10.1007/BF01418931

[7] Zucker, I.J. (1987) A Systematic Way of Converting Infinite Series into Infinite Products. Journal of Physics A, 20, L13-L17. http://dx.doi.org/10.1088/0305-4470/20/1/003

[8] Zucker, I.J. (1990) Further Relations amongst Infinite Series and Products: II. The Evaluation of Three-Dimensional Lattice Sums. Journal of Physics A, 23, 117-132. http://dx.doi.org/10.1088/0305-4470/23/2/009

[9] Kendirli, B. (2015) Evaluation of Some Convolution Sums by Quasimodular Forms. European Journal of Pure and Applied Mathematics, 8, 81-110.

[10] Kendirli, B. (2015) Evaluation of Some Convolution Sums and Representation Numbers of Quadratic Forms of Discriminant -135. British Journal of Mathematics and Computer Science, 6, 494-531. http://dx.doi.org/10.9734/BJMCS/2015/13973

[11] Kendirli, B. (2014) Evaluation of Some Convolution Sums and the Representation Numbers. Ars Combinatoria, CXVI, 65-91.

[12] Kendirli, B. (2012) Cusp Forms in $S_{4}\left(\Gamma_{0}(79)\right)$ and the Number of Representations of Positive Integers by Some Direct Sum of Binary Quadratic Forms with Discriminant -79. Bulletin of the Korean Mathematical Society, 49, 529-572. http://dx.doi.org/10.4134/BKMS.2012.49.3.529

[13] Kendirli, B. (2012) Cusp Forms in $S_{4}\left(\Gamma_{0}(47)\right)$ and the Number of Representations of Positive Integers by Some Direct Sum of Binary Quadratic Forms with Discriminant -47. International Journal of Mathematics and Mathematical Sciences, 2012, Article ID: 303492.

[14] Kendirli, B. (2012) The Bases of $M_{4}\left(\Gamma_{0}(71)\right), M_{6}\left(\Gamma_{0}(71)\right)$ and the Number of Representations of Integers. $M a-$ thematical Problems in Engineering, 2013, Article ID: 695265.

[15] Alaca, A., Alaca, S. and Williams, K.S. (2006) On the Two-Dimensional Theta Functions of Borweins. Acta Arithmetica, 124, 177-195. http://dx.doi.org/10.4064/aa124-2-4

[16] Alaca, A., Alaca, S. and Williams, K.S. (2006) Evaluation of the convolution sums $\sum_{l+12 m=n} \sigma(l) \sigma(m)$ and $\sum_{3 l+4 m=n} \sigma(l) \sigma(m)$. Advances in Theoretical and Applied Mathematics, 1, 27-48.

[17] Gordon, B. and Robins, S. (1995) Lacunarity of Dedekind $\eta$-Products. Glasgow Mathematical Journal, 37, 1-14. http://dx.doi.org/10.1017/S0017089500030329

[18] Diamond, F. and Shurman, J. (2005) A First Course in Modular Forms. Springer Graduate Texts in Mathematics 228. Springer, New York. 